\title{
NEW HUBBLE SPACE TELESCOPE OBSERVATIONS OF HEAVY ELEMENTS IN FOUR METAL-POOR STARS*
}

\author{
Ian U. Roederer ${ }^{1}$, James E. Lawler ${ }^{2}$, Jennifer S. Sobeck ${ }^{3}$, Timothy C. Beers ${ }^{4,5,6}$, John J. Cowan ${ }^{7}$, Anna Frebel ${ }^{8}$, \\ Inese I. Ivans ${ }^{9}$, Hendrik Schatz ${ }^{5,6,10}$, Christopher SNeden $^{11}$, AND IAN B. ThOMPSON ${ }^{1}$ \\ ${ }^{1}$ Carnegie Observatories, Pasadena, CA 91101, USA \\ ${ }^{2}$ Department of Physics, University of Wisconsin, Madison, WI 53706, USA \\ ${ }^{3}$ Department of Astronomy \& Astrophysics, University of Chicago, Chicago, IL 60637, USA \\ ${ }^{4}$ National Optical Astronomy Observatory, Tucson, AZ 85719, USA \\ ${ }^{5}$ Department of Physics \& Astronomy, Michigan State University, E. Lansing, MI 48824, USA \\ ${ }^{6}$ Joint Institute for Nuclear Astrophysics, Michigan State University, E. Lansing, MI 48824, USA \\ ${ }^{7}$ Homer L. Dodge Department of Physics and Astronomy, University of Oklahoma, Norman, OK 73019, USA \\ ${ }^{8}$ Massachusetts Institute of Technology, Kavli Institute for Astrophysics and Space Research, Cambridge, MA 02139, USA \\ ${ }^{9}$ Department of Physics and Astronomy, University of Utah, Salt Lake City, UT 84112, USA \\ ${ }^{10}$ National Superconducting Cyclotron Laboratory, Michigan State University, East Lansing, MI 48824, USA \\ ${ }_{11}^{11}$ Department of Astronomy, University of Texas at Austin, Austin, TX 78712, USA \\ Received 2012 January 3; accepted 2012 October 23; published 2012 November 21
}

\begin{abstract}
Elements heavier than the iron group are found in nearly all halo stars. A substantial number of these elements, key to understanding neutron-capture nucleosynthesis mechanisms, can only be detected in the near-ultraviolet. We report the results of an observing campaign using the Space Telescope Imaging Spectrograph on board the Hubble Space Telescope to study the detailed heavy-element abundance patterns in four metal-poor stars. We derive abundances or upper limits from 27 absorption lines of 15 elements produced by neutron-capture reactions, including seven elements (germanium, cadmium, tellurium, lutetium, osmium, platinum, and gold) that can only be detected in the near-ultraviolet. We also examine 202 heavy-element absorption lines in ground-based optical spectra obtained with the Magellan Inamori Kyocera Echelle Spectrograph on the Magellan-Clay Telescope at Las Campanas Observatory and the High Resolution Echelle Spectrometer on the Keck I Telescope on Mauna Kea. We have detected up to 34 elements heavier than zinc. The bulk of the heavy elements in these four stars are produced by $r$-process nucleosynthesis. These observations affirm earlier results suggesting that the tellurium found in metal-poor halo stars with moderate amounts of $r$-process material scales with the rare earth and third $r$-process peak elements. Cadmium often follows the abundances of the neighboring elements palladium and silver. We identify several sources of systematic uncertainty that must be considered when comparing these abundances with theoretical predictions. We also present new isotope shift and hyperfine structure component patterns for Lu II and $\mathrm{Pb}$ I lines of astrophysical interest.
\end{abstract}

Key words: nuclear reactions, nucleosynthesis, abundances - stars: abundances - stars: individual (HD 108317 , HD 122563, HD 126238, HD 128279) - stars: Population II

Online-only material: color figures, machine-readable tables

\section{INTRODUCTION}

Thousands of high-resolution spectroscopic observations acquired over the last few decades have revealed that nearly all stars contain at least traces of elements heavier than the iron group. These elements are primarily produced by neutron-capture reactions, and their star-to-star abundance variations span several orders of magnitude. Two general reaction timescales are involved, those that are slow (the $s$-process) or rapid (the $r$-process) relative to the average $\beta$-decay times for unstable nuclei along the reaction paths. The abundance distributions are largely governed by nuclear structure and the physical conditions at the time of nucleosynthesis.

\footnotetext{
* Based on observations made with the NASA/ESA Hubble Space Telescope, obtained at the Space Telescope Science Institute, which is operated by the Association of Universities for Research in Astronomy, Inc., under NASA contract NAS 5-26555. These observations are associated with programs 8111 and 12268. This paper includes data gathered with the $6.5 \mathrm{~m}$ Magellan Telescopes located at Las Campanas Observatory, Chile. Some of the data presented herein were obtained at the W. M. Keck Observatory, which is operated as a scientific partnership among the California Institute of Technology, the University of California, and the National Aeronautics and Space Administration. The Observatory was made possible by the generous financial support of the W. M. Keck Foundation.
}

The solar system (SS) composition represents one chemical snapshot of the Galactic interstellar medium 4.5 Gyr ago. Models predict that about $51 \%$ (by mass or by number) of the heavy elements in the SS originated through $s$-process nucleosynthesis, with the remaining $49 \%$ produced by other nucleosynthesis mechanisms, primarily the $r$-process. This is revealed by comparing the SS isotopic abundance distribution with predictions from analytical models or nuclear reaction networks coupled to stellar evolution codes (e.g., Seeger et al. 1965; Cameron 1973, 1982; Käppeler et al. 1989; Arlandini et al. 1999; Bisterzo et al. 2011). These models predict the $s$-process component, which is normalized to isotopes that can only be produced by $s$-process nucleosynthesis. This distribution is subtracted from the total abundance distribution to reveal the $r$-process "residuals." Small contributions from mechanisms that produce proton-rich nuclei can be subtracted in a similar way, but this is negligible for most elements. The $r$-process residuals implicitly include contributions from all other processes.

Strontium (Sr, $Z=38)$ or barium $(\mathrm{Ba}, Z=56)$ has been detected, or not excluded based on upper limits, in nearly all metal-poor field and globular cluster stars studied (e.g., McWilliam et al. 1995; Ryan et al. 1996; Honda et al. 2004; 
Aoki et al. 2005; François et al. 2007; Cohen et al. 2008; Lai et al. 2008; Roederer et al. 2010a, 2011, 2012b; Hollek et al. 2011), including the most iron-poor star known (Frebel et al. 2005). These elements are also found in all dwarf galaxies studied, even those with tight upper limits on strontium or barium in a few stars (Fulbright et al. 2004; Koch et al. 2008; Frebel et al. 2010a). This early and widespread enrichment, presumably by some form of $r$-process nucleosynthesis, argues for a nucleosynthetic site associated with common, short-lived stars. Models and simulations of the neutrino wind of corecollapse supernovae can reproduce a number of observational details (e.g., Mathews et al. 1992; Woosley et al. 1994; Wheeler et al. 1998; Wanajo et al. 2003; Farouqi et al. 2010; Peterson 2011). There are concerns, however, that conditions favorable to the "main" component of the $r$-process - a neutron-rich wind, for example - may not actually develop (e.g., Horowitz \& Li 1999; Janka et al. 2008; Fischer et al. 2010; Hüdepohl et al. 2010; Roberts et al. 2010). Merging neutron stars or neutron star-black hole binaries are attractive candidate sites also (e.g., Freiburghaus et al. 1999; Goriely et al. 2011), though they may have difficulty producing prompt $r$-process enrichment in nearly all stellar environments (e.g., Argast et al. 2004; Wanajo \& Ishimaru 2006).

Stars in the hydrogen and helium shell-burning stage on the asymptotic giant branch are major sites of $s$-process nucleosynthesis (e.g., Busso et al. 1999), and massive stars with high metallicity may activate $s$-process reactions during the core helium and shell carbon burning stages (e.g., Raiteri et al. 1993). Many low-metallicity halo giants enriched with substantial amounts of $s$-process material are found in binary systems (e.g., McClure 1984; McClure \& Woodsworth 1990). In contrast, radial-velocity monitoring of stars with $r$-process enhancements from $+0.5 \leqslant[\mathrm{Eu} / \mathrm{Fe}] \leqslant+1.8$ demonstrates that the binary fraction of these stars is no different than other low-metallicity halo stars (about 20\%; Hansen et al. 2011). This suggests that substantial levels of $r$-process enrichment do not require the presence of a binary companion, underscoring the likelihood that the astrophysical sites of the $s$ - and $r$-process are decoupled from one another. Yet $s$-process models cannot reproduce all of the abundance characteristics of another class of stars showing significant excesses of both $s$ - and $r$-process material (Lugaro et al. 2012). Studies like these highlight the need to identify the astrophysical site or sites of $r$-process nucleosynthesis.

The distributions of $r$-process material in the SS and halo stars provide constraints on the physical conditions at these candidate sites. While the SS distribution is of great value, it exhibits several shortcomings. The SS $r$-process residual distribution is only as reliable as the SS total and $s$-process distributions. The most precise isotopic abundance measurements are derived from the study of rare and fragile CI chondrite meteorites, which have experienced the least amount of fractionation (e.g., Anders 1971). The SS $r$-process distribution represents the combined yields of many $r$-process events, so interesting but rare features may be averaged out. The detailed $r$-process patterns observed in metal-poor stars offer an independent set of constraints, but they present a different set of challenges. Not all elements can be detected in stellar spectra, and only elemental-not isotopic_-abundances are generally available. Stellar abundances are derived, not measured, and require an accurate stellar atmosphere model and various atomic data. Interpreting the derived abundance pattern as being representative of only $r$-process nucleosynthesis presents a final challenge.
Despite these challenges, the SS meteoritic and solar photospheric abundances agree remarkably well for most elements (e.g., Anders \& Grevesse 1989). The scaled SS $r$-process distribution also closely matches that observed in metal-poor halo stars strongly enriched in the $r$-process, such as CS 22892-052 (Cowan et al. 1995; Sneden et al. 2003). This similarity is of great interest, but so too are the differences from one stellar $r$-process distribution to another (e.g., McWilliam 1998; Sneden et al. 2000; Hill et al. 2002; Johnson \& Bolte 2002; Aoki et al. 2005; Barklem et al. 2005; Ivans et al. 2006; Frebel et al. 2007; Honda et al. 2007; Roederer et al. 2010a; Peterson 2011; Hansen et al. 2012).

Some elements exhibit greater sensitivity to different physical aspects of the $r$-process, so it is important to try to detect these rarely seen elements in stellar spectra. The high-resolution near-ultraviolet (NUV) capabilities of the Goddard High Resolution Spectrograph (GHRS) and the Space Telescope Imaging Spectrograph (STIS) on board the Hubble Space Telescope (HST) have been especially helpful in this regard. Without them, many heavy elements would forever remain undetected-or with marginal detections only-in $r$-process-enriched stars. These include germanium (Ge, $Z=32$ ), arsenic (As, $Z=$ 33 ), selenium ( $\mathrm{Se}, Z=34$ ), molybdenum (Mo, $Z=42$ ), cadmium $(\mathrm{Cd}, Z=48)$, tellurium (Te, $Z=52$ ), lutetium (Lu, $Z=$ 71 ), osmium (Os, $Z=76$ ), iridium ( $\mathrm{Ir}, Z=77$ ), platinum ( $\mathrm{Pt}$, $Z=78$ ), gold ( $\mathrm{Au}, Z=79)$, lead $(\mathrm{Pb}, Z=82)$, and bismuth ( $\mathrm{Bi}$, $Z=83$ ) (Cowan et al. 1996, 2002, 2005; Sneden et al. 1998, 2003; Den Hartog et al. 2005; Roederer et al. 2009, 2010b, 2012; Barbuy et al. 2011; Peterson 2011; Roederer \& Lawler 2012; Roederer 2012a). Here, we employ new NUV STIS spectra with high signal-to-noise ratios ( $/ \mathrm{Ns}$ ) to push abundance studies farther into the NUV in bright, metal-poor red giant stars.

Throughout this paper we use the standard definitions of elemental abundances and ratios. For element X, the logarithmic abundance is defined as the number of atoms of element $\mathrm{X}$ per $10^{12}$ hydrogen atoms, $\log \epsilon(\mathrm{X}) \equiv \log _{10}\left(N_{\mathrm{X}} / N_{\mathrm{H}}\right)+12.0$. For elements $\mathrm{X}$ and $\mathrm{Y}$, the logarithmic abundance ratio relative to the solar ratio of $\mathrm{X}$ and $\mathrm{Y}$ is defined as $[\mathrm{X} / \mathrm{Y}] \equiv$ $\log _{10}\left(N_{\mathrm{X}} / N_{\mathrm{Y}}\right)-\log _{10}\left(N_{\mathrm{X}} / N_{\mathrm{Y}}\right)_{\odot}$. Abundances or ratios denoted with the ionization state indicate the total elemental abundance as derived from that particular ionization state after ionization corrections have been applied. When reporting relative abundance ratios (e.g., $[\mathrm{X} / \mathrm{Fe}]$ ), these ratios are constructed by comparing the abundance of element $\mathrm{X}$ derived from the neutral species with the iron abundance derived from Fe I and abundance derived from the ionized species of element $\mathrm{X}$ with the iron abundance derived from Fe II.

\section{OBSERVATIONS}

\subsection{Target Selection}

Stars with strong or moderate levels of $r$-process material have been well studied using the GHRS and STIS. Our goal in the present study is to investigate the abundances of heavy elements in stars with modest levels of $r$-process material. We select targets that are among the brightest metal-poor stars in the sky. We restrict ourselves to red giants, where the line opacity of potentially weak absorption lines will not be completely overwhelmed by the continuous opacity, as would be the case for warmer subgiants or main-sequence stars. We select stars with a range of $r$-process enrichment levels as indicated from previous ground-based studies. Our final sample is composed of 
Table 1

Log of Observations

\begin{tabular}{|c|c|c|c|c|}
\hline Star & Program ID & Data Set & $\begin{array}{l}\text { Date } \\
\text { (UT) }\end{array}$ & $\begin{array}{c}\text { Time } \\
(\mathrm{ks})\end{array}$ \\
\hline \multicolumn{5}{|c|}{ Observations with $H S T /$ STIS } \\
\hline HD 108317 & GO-12268 & OBJQ01010-50 & 2011 Jun 11 & 12.9 \\
\hline HD 108317 & GO-12268 & OBJQ02010-50 & 2011 Jul 13 & 12.9 \\
\hline HD 108317 & GO-12268 & OBJQ03010-50 & 2011 Jul 15 & 12.9 \\
\hline HD 122563 & GO-8111 & O5EL01010-40 & 1999 Jul 29 & 10.4 \\
\hline HD 122563 & GO-12268 & OBJQ09010-40 & 2011 Jul 24 & 9.9 \\
\hline HD 122563 & GO-12268 & OBJQ10010-40 & 2011 Aug 06 & 9.9 \\
\hline HD 126238 & GO-12268 & OBJQ07010-50 & 2011 Jul 20 & 13.4 \\
\hline HD 126238 & GO-12268 & OBJQ08010-50 & 2011 Jul 21 & 13.4 \\
\hline HD 128279 & GO-12268 & OBJQ04010-50 & 2011 Aug 24 & 13.0 \\
\hline HD 128279 & GO-12268 & OBJQ05010-50 & 2011 Aug 26 & 13.0 \\
\hline HD 128279 & GO-12268 & OBJQ06010-50 & 2011 Sep 05 & 13.0 \\
\hline \multicolumn{5}{|c|}{ Observations with Magellan/MIKE } \\
\hline HD 108317 & $\ldots$ & $\ldots$ & $2009 \mathrm{Feb} 21$ & 0.12 \\
\hline HD 122563 & $\ldots$ & $\ldots$ & 2009 Feb 19 & 0.03 \\
\hline HD 126238 & $\ldots$ & $\ldots$ & 2009 Sep 04 & 0.10 \\
\hline HD 128279 & $\ldots$ & $\ldots$ & 2004 Jun 22 & 0.02 \\
\hline HD 128279 & $\ldots$ & $\ldots$ & 2004 Jul 14 & 0.20 \\
\hline HD 128279 & $\ldots$ & $\ldots$ & 2004 Jul 23 & 0.90 \\
\hline HD 128279 & $\cdots$ & $\cdots$ & 2005 May 31 & 0.24 \\
\hline \multicolumn{5}{|c|}{ Observations with Keck/HIRES } \\
\hline HD 108317 & $\ldots$ & $\ldots$ & 1999 Apr 21 & 3.30 \\
\hline HD 122563 & $\ldots$ & $\ldots$ & 1999 Apr 21 & 0.60 \\
\hline HD 126238 & $\ldots$ & $\ldots$ & 1999 Apr 21 & 2.04 \\
\hline HD 128279 & $\ldots$ & $\ldots$ & 1999 Apr 22 & 2.40 \\
\hline
\end{tabular}

four stars. HD 122563 is the brightest metal-poor star in the sky and has been observed previously with STIS. We include it in our program to increase the $\mathrm{S} / \mathrm{N}$ at shorter wavelengths. HD 126238 has been observed previously with the GHRS, though with only very limited wavelength coverage. These are the first high-resolution NUV spectral observations of HD 108317 and HD 128279.

\subsection{HST/STIS Spectra}

In Program GO-12268, we obtained new STIS (Kimble et al. 1998; Woodgate et al. 1998) observations using the E230M echelle grating, centered on $\lambda 2707$, and the NUV Multianode Microchannel Array (MAMA) detector. The 0.'06 $\times 0$ 0.2 slit yields a $\sim 2$ pixel resolving power $(R \equiv \lambda / \Delta \lambda) \sim 30,000$. This setup produces wavelength coverage from $\lambda \lambda 2280$ to 3115 in a single exposure. Table 1 presents the log of observations acquired with STIS. The observations are taken in a standard sequence that includes acquisition and peak-up images to center the star on the narrow slit. These observations are then reduced and calibrated using the standard calstis pipeline.

We also use existing STIS spectra of HD 122563 to supplement our new observations. These observations were taken as part of Program GO-8111, and they have been discussed in detail in Cowan et al. (2005). These spectra were taken with the same instrument setup and have been co-added with the new observations.

After STIS was restored during HST Servicing Mission 4 in 2009, the NUV MAMA detector exhibited a significantly elevated dark current. This is likely caused by charged particles from the South-Atlantic Anomaly exciting meta-stable states in the detector impurities. When these states become thermally excited, they decay to the ground state by producing ultraviolet
(UV) photons that are detected by the MAMA photocathode (Kimble et al. 1998; Zheng et al. 2011). This reduces the S/N that can be attained to levels lower than would otherwise be expected from Poisson statistics. The $\mathrm{S} / \mathrm{N}$ estimates listed in Table 2 reflect this reality. The dark current is significantly lower in the STIS spectra of HD 122563 taken from Program GO-8111, and Poisson statistics dominate the $\mathrm{S} / \mathrm{N}$ budget in these observations. Nevertheless, our new observations of HD 122563 increase the $\mathrm{S} / \mathrm{N}$ of the previous observations by $50 \%-60 \%$.

\subsection{Magellan/MIKE Spectra}

We supplement our STIS observations with high-resolution optical spectra obtained from the ground using the Magellan Inamori Kyocera Echelle (MIKE) spectrograph (Bernstein et al. 2003) on the Magellan-Clay Telescope at Las Campanas Observatory. The MIKE spectra were taken with the $0^{\prime \prime} 7 \times 5 . \prime 0$ slit, yielding a resolving power of $R \sim 41,000$ in the blue and $R \sim$ 35,000 in the red, split by a dichroic around $\lambda 4950$. This setup achieves complete wavelength coverage from $\lambda \lambda 3350$ to 9150 . Data reduction, extraction, and wavelength calibration were performed using the current version of the MIKE data reduction pipeline (written by D. Kelson; see also Kelson 2003). Coaddition and continuum normalization were performed within the Image Reduction and Analysis Facility (IRAF) environment, and final $\mathrm{S} / \mathrm{N}$ estimates are listed in Table 2.

\subsection{Keck/HIRES Spectra}

To fill in the remaining spectral gap between the STIS and MIKE spectra, we use data collected with the High Resolution Echelle Spectrometer (HIRES; Vogt et al. 1994) on the Keck I Telescope on Mauna Kea. These spectra were taken with the 0 .'86 $\times 7$ ". 0 slit, yielding a resolving power of $R \sim 45,000$. This setup achieves complete wavelength coverage from $\lambda \lambda 3120$ to 4640. Results from some of these spectra have been published previously in Cowan et al. (2005), and we refer the interested reader to this paper for further details. $\mathrm{S} / \mathrm{N}$ estimates are given in Table 2.

\section{NEUTRON-CAPTURE TRANSITIONS IN THE NUV}

We begin our search for useful transitions of heavy elements using line lists published in previous investigations. We supplement these lists with low-excitation lines provided in the compilation by Morton (2000). We also include low-excitation lines or lines with relatively large transition probabilities. We include possible transitions of both the neutral and first ion species, though usually one ionization state of each element dominates in these cool stellar atmospheres (see, e.g., Figure 1 of Roederer \& Lawler 2012). Any NUV transitions of elements with several optical lines that yield reliable abundances have been ignored for the purposes of this study (e.g., the rare earth elements). We retain all potentially useful zirconium ( $\mathrm{Zr}, Z=$ 40) transitions in the NUV as a control to compare the optical and NUV abundance scales.

We also search for heavy-element transitions by comparing the spectra of HD 108317 and HD 128279. These two stars have nearly identical effective temperatures and metallicities. Differing abundances of heavy elements in their atmospheres account for most of the differences in their spectra. We consider extra absorption detected in HD 108317 relative to HD 128279 possible evidence of a heavy-element transition, and we attempt to match these wavelengths with lines in the National Institute 
Table 2

Continuum Signal-to-noise Estimates

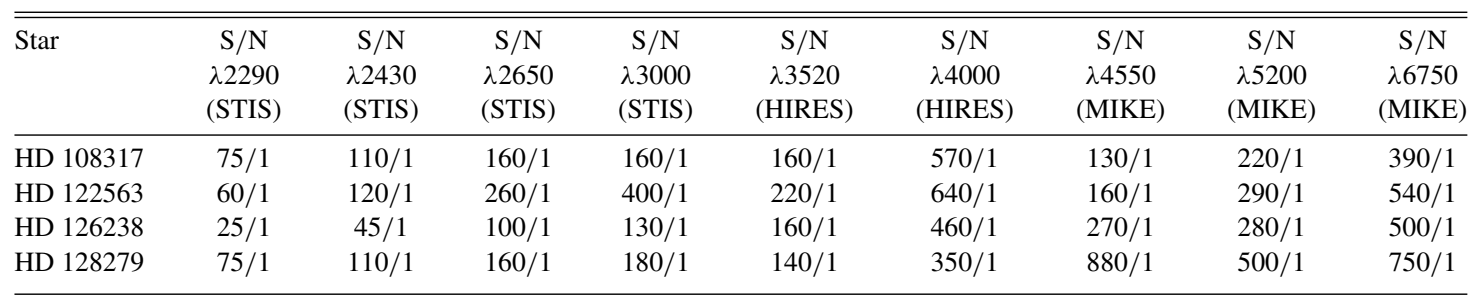

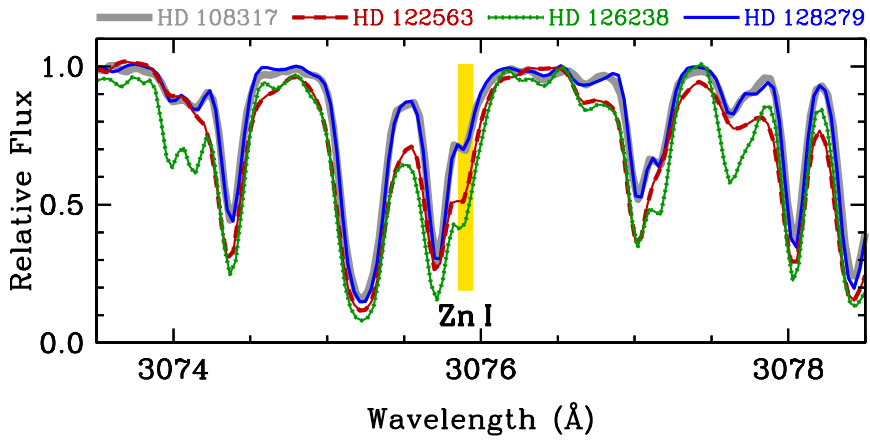

Figure 1. STIS spectra of all four stars around the Zn I $\lambda 3075$ line, marked by the shaded region.

(A color version of this figure is available in the online journal.)

of Standards and Technology (NIST) Atomic Spectra Database (Ralchenko et al. 2011) or other laboratory studies. This technique identified several lines of interstellar absorption toward HD 128279 (see Section 6) and yielded a few heavy-element transitions worth pursuing further.

Our search produced an initial set of approximately 300 transitions between $\lambda \lambda 2280$ and 3115 for further consideration. We refine this list by examining the spectra of HD 108317 and HD 128279 at the wavelength of each transition. In a few cases no absorption is detected at all. Many potentially useful heavyelement transitions lie in regions that are hopelessly blended with absorption from more abundant species, usually neutral or first ion iron group elements. We discard these transitions from our list. This step reduces our initial list by about half.

We next generate a synthetic spectrum covering $\pm 3 \AA$ around each line of interest to determine whether the absorption is due to the species of interest. We produce line lists from the Kurucz $\&$ Bell (1995) atomic and molecular lists, updating them with laboratory $\log (g f)$ values when known. We include hyperfine splitting (hfs) structure for odd- $Z$ iron group elements. This step reveals that many of the potential heavy-element transitions are far too weak or too blended to be reliably detected in our sample.

We are left with 27 lines worthy of close scrutiny. These lines are discussed in detail in Appendix A. In addition to a handful of $\mathrm{Zr}$ II lines, our STIS spectra reveal absorption lines due to the heavy elements zinc $(\mathrm{Zn}, Z=30)$, germanium, niobium $(\mathrm{Nb}$, $Z=41$ ), cadmium, tellurium, lutetium, hafnium (Hf, $Z=72$ ), osmium, iridium, platinum, gold, and lead. The spectral regions surrounding lines of each of these elements are shown for all four stars in Figures 1-7. In addition, we derive upper limits from non-detections of molybdenum (Mo II; we can detect Mo I in the ground-based spectra) and bismuth.

In Table 3 we present a list of 229 lines examined in each star, including the 27 lines examined in our new STIS spectra. This table includes the line wavelength, species identification, excitation potential (EP) of the lower level of the transition,
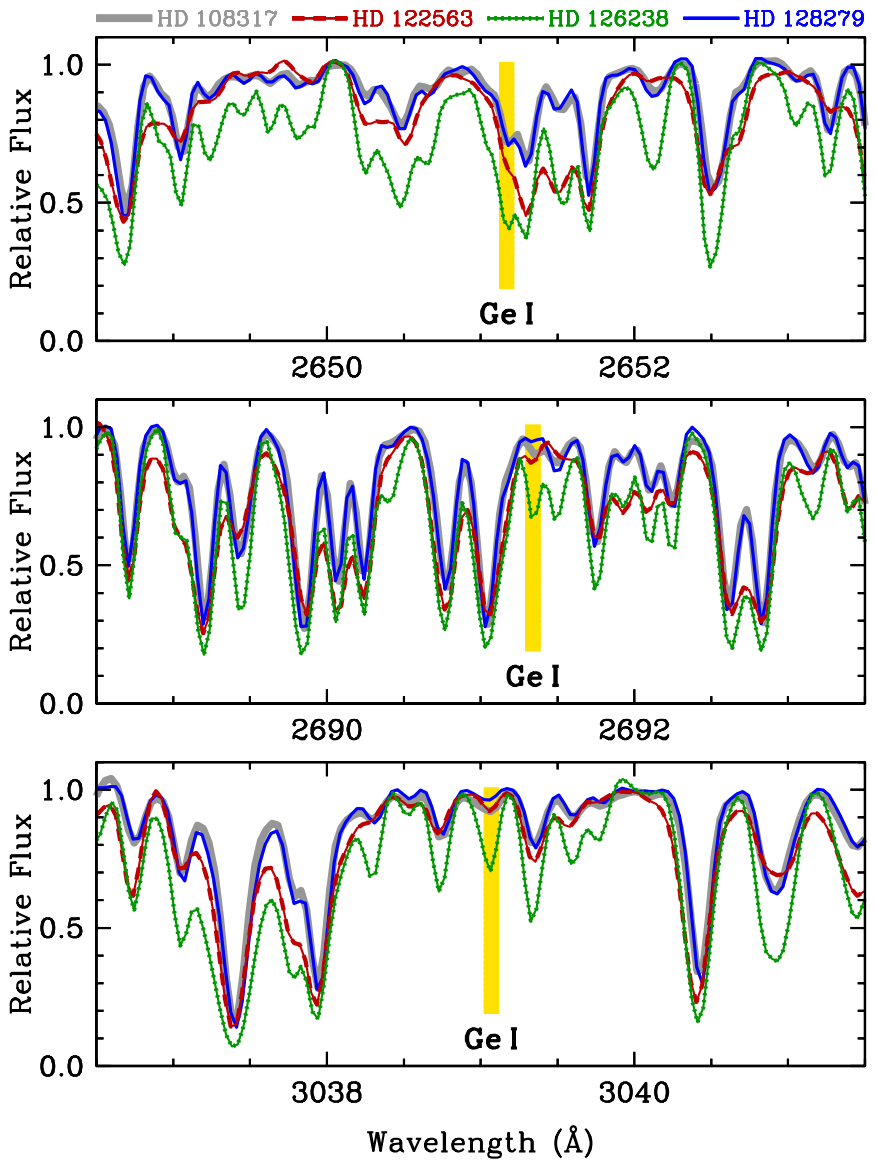

Figure 2. STIS spectra of all four program stars around the Ge I $\lambda \lambda 2651,2691$, and 3039 lines.

(A color version of this figure is available in the online journal.)

$\log (g f)$ value, and references to the source of the $\log (g f)$ value and any hfs structure or isotope shifts (ISs) included in the syntheses.

The rare earth elements from lanthanum (La, $Z=57)$ through lutetium are exclusively detected as first ions in the atmospheres of metal-poor red giant stars. The first ionization potentials of these elements are relatively low, ranging from 5.43 to $6.25 \mathrm{eV}$. In contrast, the elements at the third $r$-process peak (osmium through gold) and lead have significantly higher first ionization potentials, ranging from 7.42 to $9.23 \mathrm{eV}$. The neutral species of these elements are usually detected, though the ionized species may also be present in substantial amounts. Strontium through cadmium and tellurium have gradually increasing first ionization potentials from 5.69 to $9.01 \mathrm{eV}$. Strontium, yttrium, zirconium, and niobium are detected as first ions in these stars, while molybdenum through cadmium and tellurium are detected 

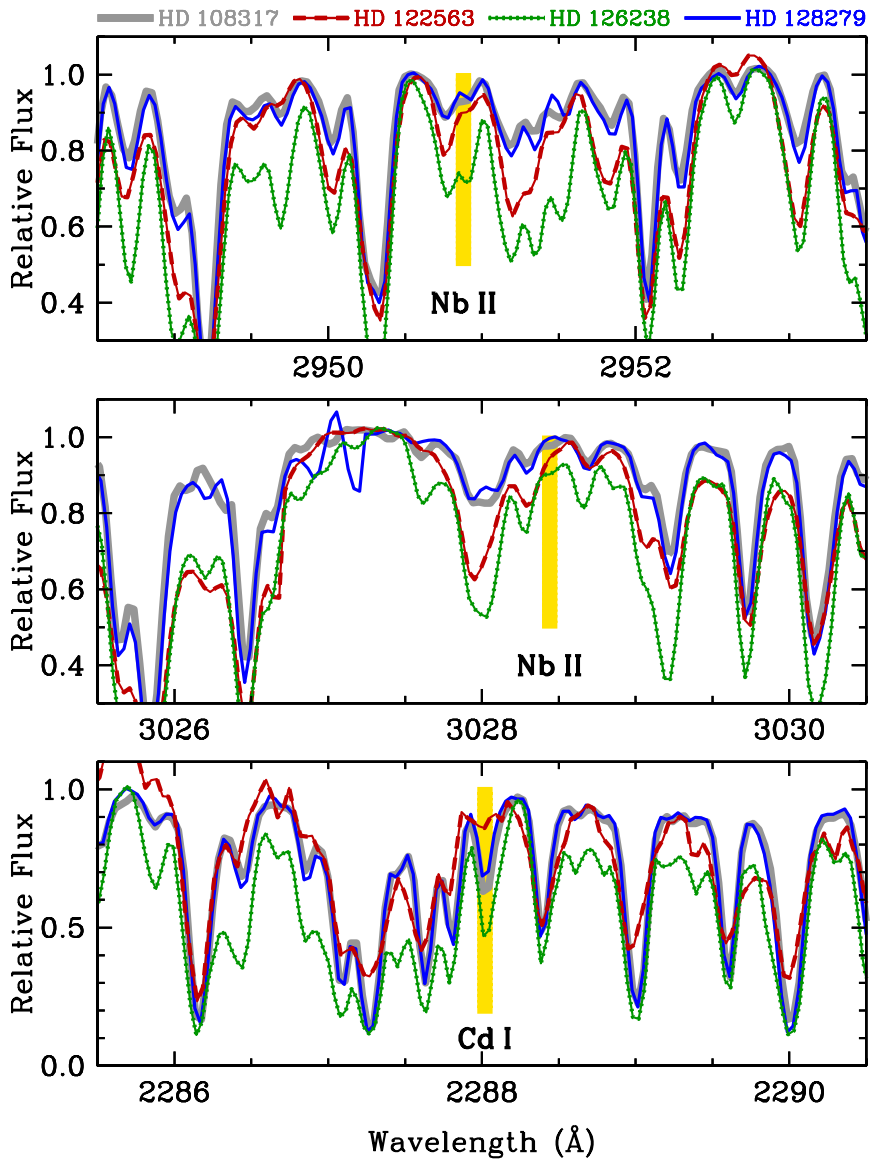

Figure 3. STIS spectra of all four program stars around the Nb II $\lambda \lambda 2950$ and 3028 lines and the $\mathrm{Cd}$ I $\lambda 2288$ line.

(A color version of this figure is available in the online journal.)

in their neutral states. Germanium has a high first ionization potential, $7.90 \mathrm{eV}$, and is also detected in the neutral state.

\section{IRON EQUIVALENT WIDTHS}

We measure equivalent widths (EWs) of $\mathrm{Fe} I$ and II lines from our MIKE spectra using a semi-automatic routine that fits Voigt absorption line profiles to continuum-normalized spectra. The complete list of EW measurements is given in Table 4, which is available only in the online version of the journal. A sample is shown in the printed edition to demonstrate its form and content.

\section{MODEL ATMOSPHERES}

Our target stars are all relatively nearby at distances of 150-300 pc. The Hipparcos satellite (Perryman et al. 1997) measured parallaxes, $\pi$, to each of these stars to better than $20 \%$, as given by the reduction validated by van Leeuwen (2007). Table 5 presents the Hipparcos parallax, distance, $V$ magnitude (from SIMBAD), $K$ magnitude (from the Two Micron All Sky Survey; Skrutskie et al. 2006), reddening (from the Schlegel et al. 1998 dust maps, modified in cases of high reddening according to the prescription given in Bonifacio et al. 2000), and de-reddened $V-K$ color (assuming the extinction coefficients of Cardelli et al. 1989). We use the $V-K$ color-temperature relation derived by Alonso et al. (1999b) to compute an initial estimate of effective temperature $\left(T_{\text {eff }}\right)$, where the quoted statistical uncertainties account for uncertainties in the photometry and the scatter in the color $-T_{\text {eff }}$ relation. We compute initial estimates
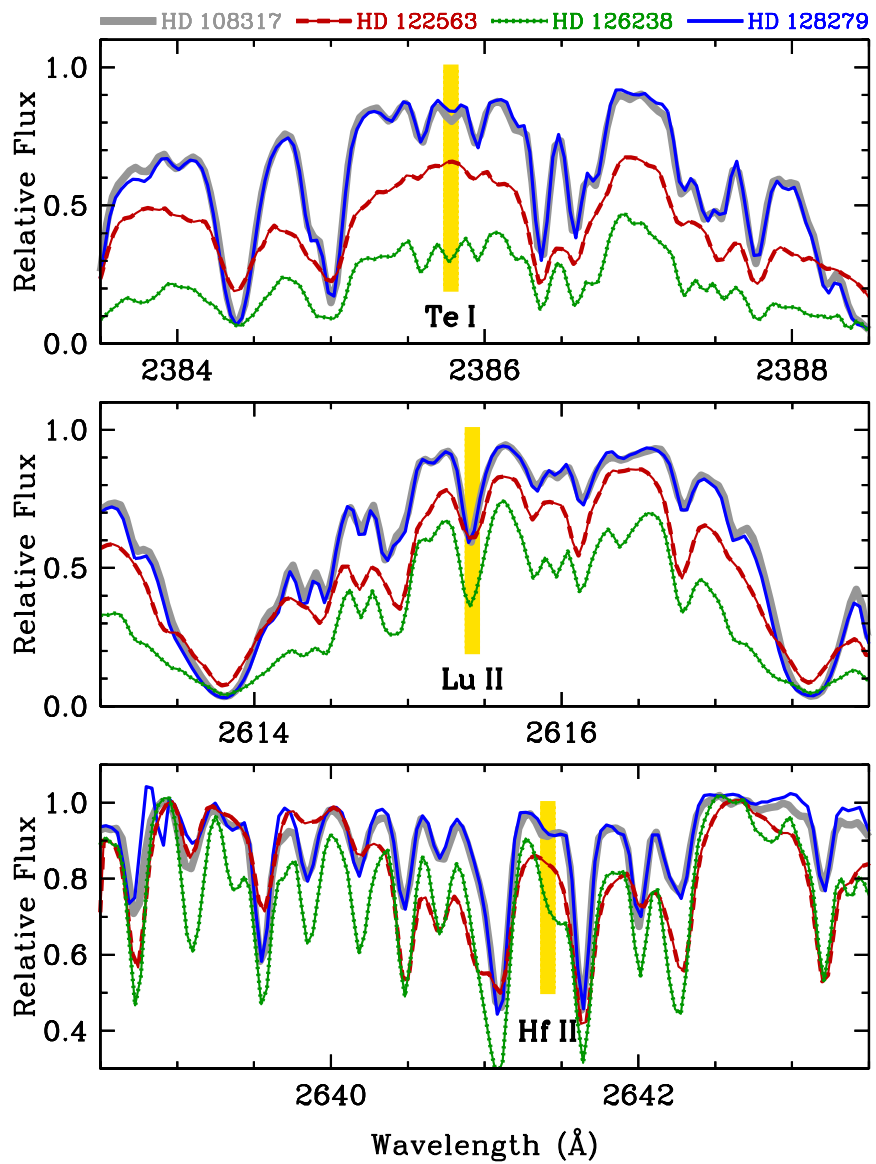

Figure 4. STIS spectra of all four program stars around the Te I $\lambda 2385$ line, the Lu II $\lambda 2615$ line, and the Hf II $\lambda 2641$ line.

(A color version of this figure is available in the online journal.)
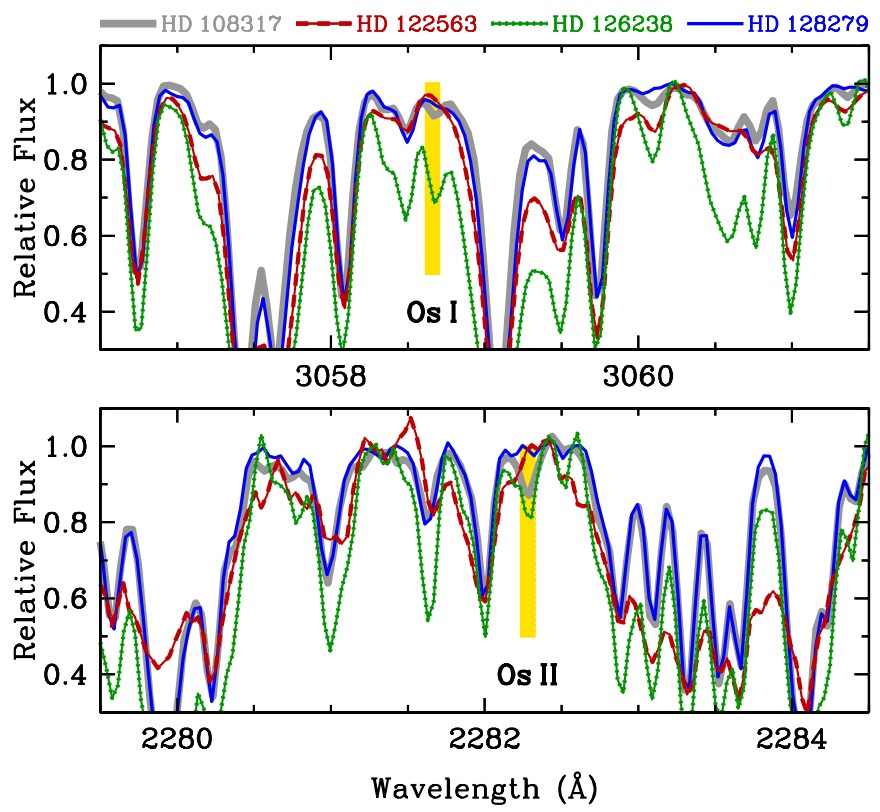

Figure 5. STIS spectra of all four program stars around the Os I $\lambda 3058$ line and the Os II $\lambda 2282$ line.

(A color version of this figure is available in the online journal.)

of the surface gravities $(\log g)$ using these data, bolometric corrections from Alonso et al. (1999a), an assumed stellar mass of $0.8 M_{\odot}$, and solar parameters $M_{\mathrm{bol}}=4.74, \log g_{\odot}=$ 4.44 , and $T_{\text {eff } \odot}=5780 \mathrm{~K}$. The quoted statistical uncertainties 

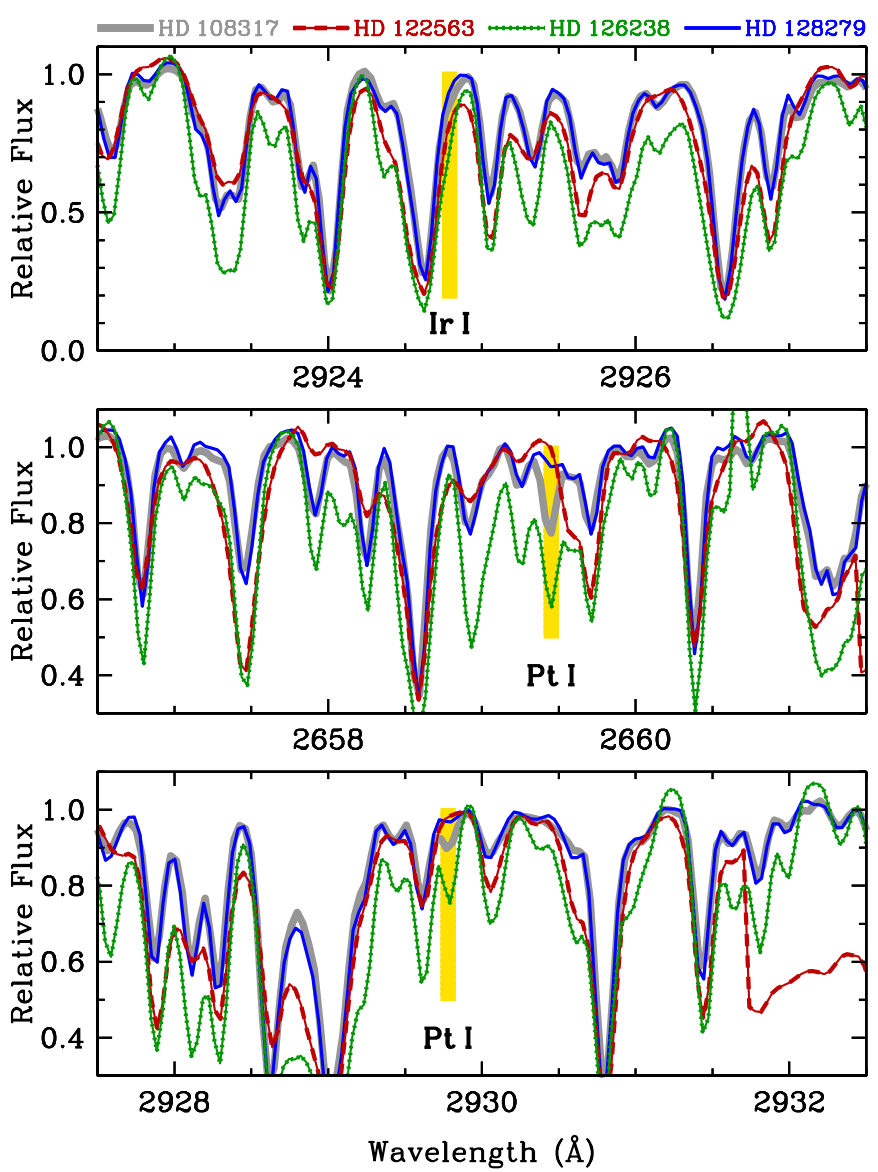

Figure 6. STIS spectra of all four program stars around the Ir I $\lambda 2924$ line and the Pt I $\lambda \lambda 2659$ and 2929 lines.

(A color version of this figure is available in the online journal.)

on $\log g$ in Table 5 reflect the uncertainties of the input quantities.

We derive abundances of iron from Fe I and II using our measured EWs, interpolations among the $\alpha$-enhanced grid of ATLAS9 model atmospheres (Castelli \& Kurucz 2003), and the latest version of the analysis code MOOG (Sneden 1973). This version of MOOG includes the contribution of Rayleigh scattering from atomic $\mathrm{H} \mathrm{I}$ in the source function (Sobeck et al. 2011).

For reasonable estimates of the microturbulent velocity $\left(v_{t}\right)$, each initial temperature estimate produces a strong correlation of iron abundance (derived from Fe I) with EP, implying that the photometric temperatures are too warm by several hundred $\mathrm{K}$. We adjust the temperature and microturbulent velocity to minimize abundance correlations between EP and line strength, $\log (\mathrm{EW} / \lambda)$, respectively. We set the overall model metallicity to the iron abundance derived from Fe II. We then recompute $\log g$ as described above and iterate until all four parameters converge. Our adopted model parameters are listed in Table 6. This method does not enforce iron ionization balance, so the iron abundance derived from Fe I lines is not necessarily equal to the iron abundance derived from Fe II lines.

A recent measurement of the radius of HD 122563 results in a derived $T_{\text {eff }}(4598 \pm 41 \mathrm{~K}$; Creevey et al. 2012) that is intermediate between the photometric $(4680 \pm 65 \mathrm{~K})$ and spectroscopic $(4450 \mathrm{~K})$ values. The corresponding value of $\log g(1.60 \pm 0.04$; Creevey et al.) agrees with the value derived from the Hipparcos parallax $(1.58 \pm 0.13)$, both of which are slightly higher than
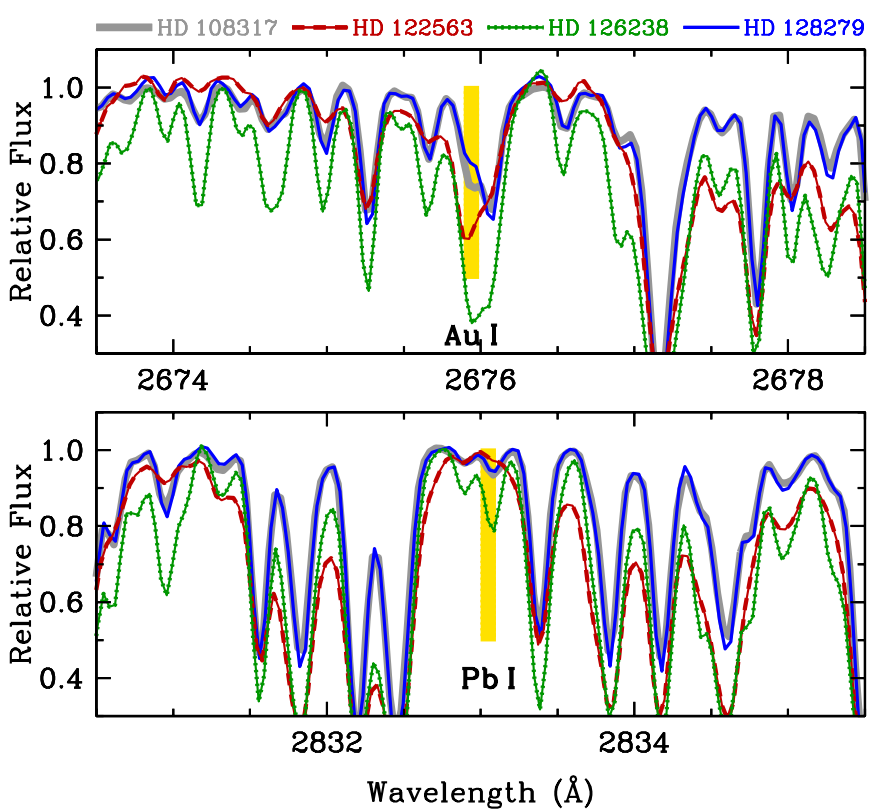

Figure 7. STIS spectra of all four program stars around the Au I $\lambda 2675$ line and the $\mathrm{Pb}$ I $\lambda 2833$ line.

(A color version of this figure is available in the online journal.)

our derived value (1.37). This comparison and a comparison of the values in Tables 5 and 6 suggest that systematic uncertainties in $T_{\text {eff }}$ may be $\approx 4 \%-6 \%$ and systematic uncertainties in $\log g$ may be $\approx 20 \%$. This offset between photometrically and spectroscopically determined model parameters is well known (e.g., Frebel et al. 2010b). Our work simply reaffirms this offset.

\section{INTERSTELLAR ABSORPTION}

In principle, overestimation of the reddening can account for the large differences between the photometric and spectroscopic temperatures. The Schlegel et al. (1998) dust maps predict significant color excess at infinity, $E(B-V)>0.1$, along the lines of sight to HD 126238 and HD 128279. These two stars are located at relatively low Galactic latitude $\left(b<30^{\circ}\right)$ and may be within the reddening layer. They also have large temperature adjustments $(-320$ and $-470 \mathrm{~K}$, respectively). We detect probable interstellar absorption from transitions from the ground states of $\mathrm{Na}$ I $(\lambda \lambda 5889.95,5895.92), \mathrm{K}_{\mathrm{I}}(\lambda 7698.96)$, Mn II $(\lambda \lambda 2593.73,2605.69)$, and Fe II $(\lambda \lambda 2343.50,2373.74$, $2382.04,2585.88,2599.40)$ in the spectrum of HD 128279. These interstellar lines all have a velocity offset of about $+60 \mathrm{~km} \mathrm{~s}^{-1}$ relative to the stellar lines. We also detect interstellar absorption in the spectrum of HD 126238 due to Na I. The lines of sight to HD 108317 and HD 122563 are less reddened, $E(B-V)<0.03$, according to the maps of Schlegel et al. (1998). These stars are located at higher Galactic latitude $\left(b>65^{\circ}\right)$ and have lower temperature corrections $(-160 \mathrm{~K}$ and $-230 \mathrm{~K}$, respectively).

We use the interstellar $\mathrm{Na}$ I lines to estimate the reddening toward these stars. We make an approximate removal of the telluric water vapor lines in this region using a smoothed version of the telluric spectrum presented by Hinkle et al. (2000). We do not detect $\mathrm{Na}$ I interstellar absorption toward HD 108317 and HD 122563. We can only estimate the interstellar absorption from the $\mathrm{Na}$ I $\lambda 5895$ line toward HD 126238 due to residual telluric contamination of the $\lambda 5889$ line. Both lines are cleanly detected toward HD 128279, but they are not resolved in 
Table 3

Atomic Data and Line-by-line Abundances

\begin{tabular}{|c|c|c|c|c|c|c|c|c|}
\hline Species & $\begin{array}{c}\lambda \\
(\AA)\end{array}$ & $\begin{array}{c}\mathrm{EP} \\
(\mathrm{eV})\end{array}$ & $\log (g f)$ & Ref. & $\begin{array}{c}\log \epsilon \\
\text { HD } 108317\end{array}$ & $\begin{array}{c}\log \epsilon \\
\text { HD } 122563\end{array}$ & $\begin{array}{c}\log \epsilon \\
\text { HD } 126238\end{array}$ & $\begin{array}{c}\log \epsilon \\
\text { HD } 128279\end{array}$ \\
\hline $\mathrm{Cu}$ & 3247.54 & 0.00 & -0.06 & 1 & +0.67 & $\ldots$ & $\ldots$ & +0.85 \\
\hline $\mathrm{Cu} \mathrm{I}$ & 3273.96 & 0.00 & -0.36 & 1 & +0.73 & +0.10 & +1.53 & +0.73 \\
\hline $\mathrm{Cu}$ I & 5105.54 & 1.39 & -1.50 & 2 & $<+1.38$ & $<+0.48$ & +1.21 & $<+1.08$ \\
\hline Zn I & 3075.90 & 0.00 & -3.85 & 3 & +2.19 & +1.76 & +2.76 & +2.23 \\
\hline Zn I & 4680.14 & 4.01 & -0.85 & 3 & $<+2.68$ & +2.07 & +2.64 & $\ldots$ \\
\hline Zn I & 4722.16 & 4.03 & -0.37 & 3 & +2.29 & +1.94 & +2.59 & +2.14 \\
\hline Zn I & 4810.54 & 4.08 & -0.15 & 3 & +2.23 & +2.00 & +2.62 & +2.17 \\
\hline Ge I & 2651.17 & 0.17 & +0.02 & 4 & +0.27 & -0.73 & $\ldots$ & +0.25 \\
\hline Ge I & 2691.34 & 0.07 & -0.70 & 4 & +0.07 & -1.05 & $+0.37:$ & -0.22 \\
\hline Ge I & 3039.07 & 0.88 & +0.07 & 4 & +0.16 & -0.87 & +0.73 & -0.13 \\
\hline $\mathrm{Rb} I$ & 7800.27 & 0.00 & +0.14 & 4 & $<+1.40$ & $<+1.20$ & $<+1.70$ & $<+1.60$ \\
\hline SrI & 4607.33 & 0.00 & +0.28 & 5 & $\ldots$ & -0.62 & +0.48 & $\ldots$ \\
\hline Sr II & 4077.71 & 0.00 & +0.15 & 4 & +0.55 & +0.02 & $\ldots$ & -0.14 \\
\hline Sr II & 4161.79 & 2.94 & -0.47 & 4 & $\ldots$ & +0.02 & +0.98 & $\ldots$ \\
\hline Sr II & 4215.52 & 0.00 & -0.17 & 4 & +0.41 & -0.09 & $\ldots$ & -0.22 \\
\hline Y II & 3549.00 & 0.13 & -0.29 & 6 & -0.22 & -0.70 & -0.02 & -0.70 \\
\hline$Y_{\text {II }}$ & 3584.51 & 0.10 & -0.42 & 6 & -0.28 & $\ldots$ & +0.01 & $\ldots$ \\
\hline$Y_{\text {II }}$ & 3600.73 & 0.18 & +0.34 & 6 & -0.38 & -0.92 & -0.06 & -0.86 \\
\hline$Y_{\text {II }}$ & 3601.92 & 0.10 & -0.15 & 6 & -0.38 & $\ldots$ & $\ldots$ & -0.80 \\
\hline $\mathrm{Y}_{\mathrm{II}}$ & 3611.04 & 0.13 & +0.05 & 6 & -0.30 & -0.84 & +0.05 & -0.78 \\
\hline Y II & 3774.33 & 0.13 & +0.29 & 6 & $\ldots$ & -0.91 & $\ldots$ & -0.90 \\
\hline Y II & 4398.01 & 0.13 & -0.75 & 6 & $\ldots$ & -1.15 & $\ldots$ & $\ldots$ \\
\hline$Y_{\text {II }}$ & 4883.68 & 1.08 & +0.19 & 6 & -0.48 & -0.98 & -0.18 & -1.05 \\
\hline Y II & 4900.12 & 1.03 & +0.03 & 6 & -0.51 & -1.01 & $\ldots$ & $\ldots$ \\
\hline$Y_{\text {II }}$ & 5087.42 & 1.08 & -0.16 & 6 & -0.46 & -0.84 & -0.16 & -1.03 \\
\hline Y II & 5200.41 & 0.99 & -0.47 & 6 & -0.55 & -1.08 & -0.22 & $\ldots$ \\
\hline$Y_{\text {II }}$ & 5205.72 & 1.03 & -0.28 & 6 & -0.48 & -0.98 & -0.18 & $\ldots$ \\
\hline $\mathrm{Zr}$ II & 2567.64 & 0.00 & -0.17 & 7 & +0.17 & $\ldots$ & +0.17 & -0.24 \\
\hline $\mathrm{Zr}$ II & 2699.60 & 0.04 & -0.66 & 8 & +0.26 & $\ldots$ & +0.80 & +0.06 \\
\hline Zr II & 2700.13 & 0.09 & -0.08 & 7 & +0.14 & -0.68 & +0.44 & -0.21 \\
\hline $\mathrm{Zr}$ II & 2732.72 & 0.09 & -0.49 & 7 & +0.62 & $\ldots$ & $\ldots$ & +0.21 \\
\hline $\mathrm{Zr}$ II & 2758.81 & 0.00 & -0.56 & 7 & +0.29 & -0.35 & $\ldots$ & -0.03 \\
\hline Zr II & 2915.99 & 0.47 & -0.50 & 7 & +0.38 & -0.26 & +0.71 & +0.03 \\
\hline Zr II & 3054.84 & 1.01 & +0.08 & 8 & +0.38 & -0.20 & $+1.01:$ & +0.21 \\
\hline $\mathrm{Zr}$ II & 3095.07 & 0.04 & -0.84 & 7 & +0.44 & -0.35 & +0.77 & +0.03 \\
\hline Zr II & 3125.92 & 0.00 & -0.70 & 7 & +0.53 & -0.22 & $\ldots$ & $\ldots$ \\
\hline Zr II & 3129.76 & 0.04 & -0.54 & 7 & +0.47 & $\ldots$ & $\ldots$ & $\ldots$ \\
\hline $\mathrm{Zr}$ II & 3273.05 & 0.16 & +0.30 & 7 & +0.65 & -0.25 & +0.82 & -0.04 \\
\hline Zr II & 3279.26 & 0.09 & +0.12 & 7 & $+0.35:$ & $\ldots$ & $\ldots$ & -0.10 \\
\hline Zr II & 3284.71 & 0.00 & -0.37 & 7 & +0.50 & -0.16 & +0.79 & +0.02 \\
\hline $\mathrm{Zr}$ II & 3305.15 & 0.04 & -0.65 & 7 & +0.56 & $\ldots$ & $\ldots$ & $\ldots$ \\
\hline $\mathrm{Zr}$ II & 3334.62 & 0.56 & -0.69 & 7 & +0.44 & -0.04 & $\ldots$ & $\ldots$ \\
\hline $\mathrm{Zr}$ II & 3344.79 & 1.01 & -0.35 & 7 & +0.44 & $\ldots$ & $\ldots$ & $\ldots$ \\
\hline $\mathrm{Zr}$ II & 3356.09 & 0.09 & -0.39 & 8 & +0.35 & $\ldots$ & $\ldots$ & -0.04 \\
\hline Zr II & 3357.26 & 0.00 & -0.66 & 7 & +0.38 & $\ldots$ & $\ldots$ & $\ldots$ \\
\hline Zr II & 3403.68 & 1.00 & -0.60 & 7 & +0.47 & $\ldots$ & +0.82 & $\ldots$ \\
\hline $\mathrm{Zr}$ II & 3404.83 & 0.36 & -0.49 & 7 & +0.50 & $\ldots$ & $\ldots$ & +0.11 \\
\hline Zr II & 3408.08 & 0.97 & -0.66 & 7 & +0.53 & $\ldots$ & +0.76 & $\ldots$ \\
\hline Zr II & 3410.24 & 0.41 & -0.31 & 7 & +0.50 & $\ldots$ & $\ldots$ & $\ldots$ \\
\hline Zr II & 3430.53 & 0.47 & -0.16 & 7 & +0.59 & $\ldots$ & +0.64 & $\ldots$ \\
\hline Zr II & 3457.56 & 0.56 & -0.47 & 8 & +0.44 & $\ldots$ & $\ldots$ & $\ldots$ \\
\hline Zr II & 3458.93 & 0.96 & -0.48 & 7 & $\ldots$ & $\ldots$ & +0.61 & $\ldots$ \\
\hline $\mathrm{Zr}$ II & 3479.02 & 0.53 & -0.67 & 7 & +0.38 & -0.16 & +0.67 & -0.01 \\
\hline $\mathrm{Zr}$ II & 3479.39 & 0.71 & +0.18 & 7 & +0.38 & -0.19 & +0.61 & +0.02 \\
\hline $\mathrm{Zr}$ II & 3496.20 & 0.04 & +0.26 & 7 & +0.35 & $\ldots$ & $\ldots$ & -0.19 \\
\hline $\mathrm{Zr}$ II & 3505.67 & 0.16 & -0.39 & 7 & +0.44 & -0.22 & +0.79 & +0.02 \\
\hline Zr II & 3506.05 & 1.24 & -0.68 & 8 & $\ldots$ & $\ldots$ & +0.82 & $\ldots$ \\
\hline Zr II & 3525.81 & 0.36 & -0.96 & 7 & $\ldots$ & -0.04 & $\ldots$ & $\ldots$ \\
\hline ZrII & 3549.51 & 1.24 & -0.72 & 7 & $\ldots$ & $\ldots$ & +0.91 & $\ldots$ \\
\hline $\mathrm{Zr}$ II & 3551.95 & 0.09 & -0.36 & 7 & $\ldots$ & -0.16 & +0.88 & +0.11 \\
\hline Zr II & 3556.59 & 0.47 & +0.07 & 7 & +0.41 & -0.13 & $\ldots$ & +0.05 \\
\hline Zr II & 3588.31 & 0.41 & -1.13 & 7 & $\ldots$ & $\ldots$ & $\ldots$ & +0.14 \\
\hline Zr II & 3607.37 & 1.24 & -0.70 & 7 & $\ldots$ & $\ldots$ & +0.91 & $\ldots$ \\
\hline Zr II & 3751.59 & 0.97 & +0.00 & 7 & +0.47 & -0.13 & $\ldots$ & -0.03 \\
\hline $\mathrm{Zr}$ II & 3766.82 & 0.41 & -0.83 & 7 & +0.44 & -0.04 & $\ldots$ & +0.03 \\
\hline
\end{tabular}


Table 3

(Continued)

\begin{tabular}{|c|c|c|c|c|c|c|c|c|}
\hline Species & $\begin{array}{c}\lambda \\
(\AA)\end{array}$ & $\begin{array}{l}\mathrm{EP} \\
(\mathrm{eV})\end{array}$ & $\log (g f)$ & Ref. & $\begin{array}{c}\log \epsilon \\
\text { HD } 108317\end{array}$ & $\begin{array}{c}\log \epsilon \\
\text { HD } 122563\end{array}$ & $\begin{array}{c}\log \epsilon \\
\text { HD } 126238\end{array}$ & $\begin{array}{c}\log \epsilon \\
\text { HD } 128279\end{array}$ \\
\hline $\mathrm{Zr}$ II & 3836.76 & 0.56 & -0.12 & 7 & +0.41 & -0.16 & +0.58 & +0.03 \\
\hline $\mathrm{Zr}$ II & 3998.96 & 0.56 & -0.52 & 7 & +0.47 & +0.02 & +0.73 & +0.06 \\
\hline Zr II & 4048.67 & 0.80 & -0.53 & 7 & +0.39 & -0.29 & $\ldots$ & $\ldots$ \\
\hline Zr II & 4050.32 & 0.71 & -1.06 & 7 & +0.45 & -0.11 & +0.79 & -0.09 \\
\hline Zr II & 4149.20 & 0.80 & -0.04 & 7 & +0.57 & -0.20 & +0.70 & $\ldots$ \\
\hline Zr II & 4156.27 & 0.71 & -0.78 & 7 & +0.54 & -0.11 & $\ldots$ & $\ldots$ \\
\hline $\mathrm{Zr}$ II & 4161.20 & 0.71 & -0.59 & 7 & +0.54 & -0.14 & $\ldots$ & +0.00 \\
\hline Zr II & 4208.98 & 0.71 & -0.51 & 7 & +0.45 & -0.11 & +0.76 & -0.03 \\
\hline Zr II & 4613.95 & 0.97 & -1.54 & 7 & $\ldots$ & -0.10 & +0.85 & $\ldots$ \\
\hline $\mathrm{Nb}$ II & 2950.88 & 0.51 & +0.24 & 9 & -0.86 & -1.65 & -0.26 & -0.88 \\
\hline $\mathrm{Nb}$ II & 3028.44 & 0.44 & -0.20 & 9 & -0.86 & -1.47 & -0.38 & $<-0.69$ \\
\hline $\mathrm{Nb}$ II & 3215.59 & 0.44 & -0.24 & 9 & -0.86 & -1.49 & -0.39 & $<-0.51$ \\
\hline $\mathrm{Nb}$ II & 3225.47 & 0.29 & -0.01 & 9 & $\ldots$ & $\ldots$ & -0.39 & $<-0.71$ \\
\hline Mo I & 3864.10 & 0.00 & -0.01 & 10 & -0.23 & -1.22 : & +0.18 & -0.67 \\
\hline Mo II & 2871.51 & 1.54 & +0.06 & 11 & $<+0.24$ & $<-0.65$ & $<+0.43$ & $<+0.11$ \\
\hline $\mathrm{Ru} \mathrm{I}_{\mathrm{I}}$ & 3436.74 & 0.15 & +0.15 & 12 & -0.06 & $\ldots$ & $\ldots$ & $\ldots$ \\
\hline Ru I & 3498.94 & 0.00 & +0.31 & 12 & -0.03 & -1.14 & $\ldots$ & -0.63 \\
\hline Ru I & 3742.28 & 0.34 & -0.18 & 12 & $\ldots$ & $\ldots$ & +0.11 & $\ldots$ \\
\hline Ru I & 3798.90 & 0.15 & -0.04 & 12 & $\ldots$ & $-1.11:$ & +0.11 & $\ldots$ \\
\hline Ru I & 3799.35 & 0.00 & +0.02 & 12 & $\ldots$ & $-0.72:$ & +0.05 & $\ldots$ \\
\hline $\mathrm{Rh} I$ & 3396.82 & 0.00 & +0.05 & 13 & $\ldots$ & $\ldots$ & -0.46 : & $\ldots$ \\
\hline $\mathrm{Rh}_{\mathrm{I}}$ & 3434.89 & 0.00 & +0.45 & 14 & $\ldots$ & $\ldots$ & $\ldots$ & $<-0.61$ \\
\hline $\mathrm{Rh}_{\mathrm{I}}$ & 3692.36 & 0.00 & +0.17 & 13 & $-0.84:$ & $-1.73:$ & $-1.06:$ & $\ldots$ \\
\hline $\mathrm{Rh} I$ & 3700.91 & 0.19 & -0.10 & 13 & $\ldots$ & $\ldots$ & $-0.43:$ & $\ldots$ \\
\hline Pd I & 3242.70 & 0.81 & +0.07 & 15 & $\ldots$ & $\ldots$ & -0.23 & $\ldots$ \\
\hline $\operatorname{Pd} I_{1}$ & 3404.58 & 0.81 & +0.33 & 15 & -0.70 & $-1.78:$ & -0.32 & $-1.24:$ \\
\hline Pd I & 3516.94 & 0.96 & -0.21 & 15 & -0.61 : & $<-1.23$ & -0.41 & $\ldots$ \\
\hline $\mathrm{Ag}_{\mathrm{I}}$ & 3280.68 & 0.00 & -0.02 & 16 & $-1.47:$ & $<-1.95$ & $\ldots$ & $<-1.13$ \\
\hline $\mathrm{Ag}_{\mathrm{I}}$ & 3382.89 & 0.00 & -0.33 & 16 & -1.26 : & $<-1.65$ & $-1.03:$ & $\ldots$ \\
\hline $\mathrm{Cd}_{\mathrm{I}}$ & 2288.02 & 0.00 & +0.15 & 17 & $-0.87:$ & $<-2.85$ & $\ldots$ & $-1.38:$ \\
\hline Sn I & 3801.01 & 1.07 & -0.74 & 4 & $<+0.80$ & $<+0.38$ & $\ldots$ & $\ldots$ \\
\hline Te I & 2385.79 & 0.59 & -0.81 & 18 & +0.02 & $\ldots$ & $\ldots$ & -0.18 \\
\hline Ba II & 4130.65 & 2.72 & +0.52 & 19 & -0.39 & $\ldots$ & $\ldots$ & $\ldots$ \\
\hline Ba II & 4554.03 & 0.00 & +0.14 & 19 & -0.18 & -1.88 & -0.06 & -1.08 \\
\hline Ba II & 5853.67 & 0.60 & -0.91 & 19 & -0.36 & -1.68 & +0.00 & -1.02 \\
\hline $\mathrm{Ba}$ II & 6141.71 & 0.70 & -0.03 & 19 & -0.33 & $\ldots$ & $\ldots$ & -0.99 \\
\hline La II & 3794.77 & 0.24 & +0.21 & 20 & -1.13 & $\ldots$ & -0.96 & $\ldots$ \\
\hline La II & 3949.10 & 0.40 & +0.49 & 20 & -1.16 & $\ldots$ & $\ldots$ & $\ldots$ \\
\hline La II & 3988.51 & 0.40 & +0.21 & 20 & -1.10 & -2.51 & -0.87 & -1.72 \\
\hline La II & 3995.74 & 0.17 & -0.06 & 20 & -1.07 & -2.27 & -0.84 & -1.63 \\
\hline La II & 4086.71 & 0.00 & -0.07 & 20 & -1.15 & -2.61 & -0.96 & -1.51 \\
\hline La II & 4123.22 & 0.32 & +0.13 & 20 & -1.12 & $\ldots$ & $\ldots$ & -1.72 \\
\hline La II & 4322.50 & 0.17 & -0.93 & 20 & $\ldots$ & $\ldots$ & -0.90 & $\ldots$ \\
\hline La II & 4333.75 & 0.17 & -0.06 & 20 & -1.12 & $\ldots$ & $\ldots$ & $\ldots$ \\
\hline La II & 4662.50 & 0.00 & -1.24 & 20 & $\ldots$ & $\ldots$ & -0.87 & $\ldots$ \\
\hline La II & 4920.98 & 0.13 & -0.58 & 20 & $\ldots$ & $\ldots$ & -0.90 & $\ldots$ \\
\hline La II & 4921.78 & 0.24 & -0.45 & 20 & $\ldots$ & $\ldots$ & -0.87 & $\ldots$ \\
\hline La II & 5114.56 & 0.23 & -1.03 & 20 & $\ldots$ & $\ldots$ & -0.93 & $\ldots$ \\
\hline Ce II & 3999.24 & 0.30 & +0.06 & 21 & -0.68 & -1.91 : & -0.45 & $\ldots$ \\
\hline Ce II & 4042.58 & 0.49 & +0.00 & 21 & -0.76 & $\ldots$ & -0.54 & $\ldots$ \\
\hline Ce II & 4053.50 & 0.00 & -0.61 & 21 & -0.64 & $\ldots$ & -0.51 & $\ldots$ \\
\hline Ce II & 4073.47 & 0.48 & +0.21 & 21 & -0.70 & $-1.98:$ & $\ldots$ & -1.12 \\
\hline Ce II & 4083.22 & 0.70 & +0.27 & 21 & -0.64 & $\ldots$ & -0.42 & $\ldots$ \\
\hline Ce II & 4118.14 & 0.70 & +0.13 & 21 & -0.67 & $\ldots$ & -0.51 & $\ldots$ \\
\hline Ce II & 4120.83 & 0.32 & -0.37 & 21 & -0.61 & $\ldots$ & -0.39 & $\ldots$ \\
\hline Ce II & 4127.36 & 0.68 & +0.31 & 21 & -0.73 & $\ldots$ & -0.48 & $\ldots$ \\
\hline Ce II & 4137.64 & 0.52 & +0.40 & 21 & -0.70 & -1.89 & -0.45 & -1.15 \\
\hline Ce II & 4222.60 & 0.12 & -0.15 & 21 & -0.67 & -1.92 & -0.48 & -1.18 \\
\hline Ce II & 4486.91 & 0.30 & -0.18 & 21 & $\ldots$ & $\ldots$ & -0.63 & $\ldots$ \\
\hline Ce II & 4562.36 & 0.48 & +0.21 & 21 & -0.66 & -1.94 & -0.48 & $\ldots$ \\
\hline Ce II & 4572.28 & 0.68 & +0.22 & 21 & $\ldots$ & $\ldots$ & -0.51 & $\ldots$ \\
\hline Ce II & 4628.16 & 0.52 & +0.14 & 21 & $\ldots$ & -2.03 & -0.54 & $\ldots$ \\
\hline Pr II & 4062.80 & 0.42 & +0.33 & 22 & $\ldots$ & $\ldots$ & -1.07 & $\ldots$ \\
\hline Pr II & 4179.40 & 0.20 & +0.46 & 22 & -0.99 : & $\ldots$ & $\ldots$ & $\ldots$ \\
\hline Pr II & 4189.49 & 0.37 & +0.43 & 22 & $\ldots$ & $<-2.15$ & $\ldots$ & $\ldots$ \\
\hline
\end{tabular}


Table 3

(Continued)

\begin{tabular}{|c|c|c|c|c|c|c|c|c|}
\hline Species & $\begin{array}{c}\lambda \\
(\AA)\end{array}$ & $\begin{array}{l}\text { EP } \\
(\mathrm{eV})\end{array}$ & $\log (g f)$ & Ref. & $\begin{array}{c}\log \epsilon \\
\text { HD } 108317\end{array}$ & $\begin{array}{c}\log \epsilon \\
\text { HD } 122563\end{array}$ & $\begin{array}{c}\log \epsilon \\
\text { HD } 126238\end{array}$ & $\begin{array}{c}\log \epsilon \\
\text { HD } 128279\end{array}$ \\
\hline Pr II & $4429.13^{a}$ & 0.00 & -0.49 & 22 & -0.80 & $\ldots$ & $\ldots$ & $\ldots$ \\
\hline Pr II & 5322.77 & 0.48 & -0.12 & 22 & $\ldots$ & $\ldots$ & -1.10 & $\ldots$ \\
\hline Nd II & 3784.24 & 0.38 & +0.15 & 23 & -0.72 & -2.13 & -0.49 & -1.28 \\
\hline $\mathrm{Nd}$ II & 3810.48 & 0.74 & -0.14 & 23 & $\ldots$ & $\ldots$ & -0.31 & $\ldots$ \\
\hline Nd II & 3826.41 & 0.06 & -0.41 & 23 & -0.51 & $\ldots$ & -0.46 & $\ldots$ \\
\hline $\mathrm{Nd}$ II & 3838.98 & 0.00 & -0.24 & 23 & -0.78 & $\ldots$ & $\ldots$ & $\ldots$ \\
\hline Nd II & 4004.00 & 0.06 & -0.57 & 23 & -0.74 & $\ldots$ & -0.55 & $\ldots$ \\
\hline Nd II & 4012.70 & 0.00 & -0.60 & 23 & $\ldots$ & $\ldots$ & -0.46 & $\ldots$ \\
\hline Nd II & 4018.82 & 0.06 & -0.85 & 23 & $\ldots$ & $\ldots$ & -0.64 & $\ldots$ \\
\hline $\mathrm{Nd}$ II & 4021.33 & 0.32 & -0.10 & 23 & -0.77 & $\ldots$ & -0.67 & $\ldots$ \\
\hline Nd II & 4023.00 & 0.56 & +0.04 & 23 & $\ldots$ & $\ldots$ & -0.55 & $\ldots$ \\
\hline Nd II & 4051.14 & 0.38 & -0.30 & 23 & -0.74 & $\ldots$ & $\ldots$ & $\ldots$ \\
\hline $\mathrm{Nd}$ II & 4059.95 & 0.20 & -0.52 & 23 & -0.68 & $\ldots$ & -0.58 & $\ldots$ \\
\hline $\mathrm{Nd}$ II & 4069.26 & 0.06 & -0.57 & 23 & -0.68 & $\ldots$ & -0.46 & $\ldots$ \\
\hline Nd II & 4109.45 & 0.32 & +0.35 & 23 & -0.65 & -1.96 & -0.37 & -1.19 \\
\hline $\mathrm{Nd}$ II & 4133.35 & 0.32 & -0.49 & 23 & $\ldots$ & $\ldots$ & -0.46 & $\ldots$ \\
\hline Nd II & 4232.37 & 0.06 & -0.47 & 23 & -0.71 & $\ldots$ & -0.58 & -1.22 \\
\hline $\mathrm{Nd}$ II & 4446.38 & 0.20 & -0.35 & 23 & -0.76 & -2.10 & -0.58 & -1.24 \\
\hline Nd II & 4645.76 & 0.56 & -0.76 & 23 & $\ldots$ & $\ldots$ & -0.37 & $\ldots$ \\
\hline Nd II & 4706.54 & 0.00 & -0.71 & 23 & -0.76 & $\ldots$ & -0.34 & $\ldots$ \\
\hline Nd II & 4914.38 & 0.38 & -0.70 & 23 & $\ldots$ & $\ldots$ & -0.58 & $\ldots$ \\
\hline Nd II & 5092.79 & 0.38 & -0.61 & 23 & $\ldots$ & $\ldots$ & -0.61 & $\ldots$ \\
\hline Nd II & 5234.19 & 0.55 & -0.51 & 23 & $\ldots$ & $\ldots$ & -0.55 & $\ldots$ \\
\hline Nd II & 5249.58 & 0.98 & +0.20 & 23 & $\ldots$ & $\ldots$ & -0.58 & $\ldots$ \\
\hline Nd II & 5255.51 & 0.20 & -0.67 & 23 & $\ldots$ & $\ldots$ & -0.52 & $\ldots$ \\
\hline Nd II & 5293.16 & 0.82 & +0.10 & 23 & $\ldots$ & $\ldots$ & -0.61 & $\ldots$ \\
\hline Nd II & 5319.81 & 0.55 & -0.14 & 23 & $\ldots$ & $\ldots$ & -0.58 & $\ldots$ \\
\hline Sm II & 3568.27 & 0.48 & +0.29 & 24 & -0.97 & $\ldots$ & $\ldots$ & -1.36 \\
\hline Sm II & 3706.75 & 0.48 & -0.60 & 24 & $\ldots$ & $\ldots$ & $-0.83:$ & $\ldots$ \\
\hline Sm II & 3896.97 & 0.04 & -0.67 & 24 & -1.06 & $\ldots$ & -0.86 & $\ldots$ \\
\hline Sm II & 4318.93 & 0.28 & -0.25 & 24 & -1.05 & -2.21 & -0.86 & $\ldots$ \\
\hline Sm II & 4424.34 & 0.48 & +0.14 & 24 & $-1.25:$ & $\ldots$ & $\ldots$ & $\ldots$ \\
\hline Sm II & 4434.32 & 0.38 & -0.07 & 24 & $-1.01:$ & $-2.38:$ & -0.92 & -1.61 \\
\hline Sm II & 4467.34 & 0.66 & +0.15 & 24 & $\ldots$ & -2.41 & -0.95 & $\ldots$ \\
\hline Sm II & 4472.41 & 0.18 & -0.96 & 24 & $\ldots$ & $\ldots$ & -1.04 & $\ldots$ \\
\hline Sm II & 4519.63 & 0.54 & -0.35 & 24 & $\ldots$ & $\ldots$ & -1.04 & $\ldots$ \\
\hline Sm II & 4523.91 & 0.43 & -0.39 & 24 & $\ldots$ & $\ldots$ & -1.10 & $\ldots$ \\
\hline Sm II & 4537.94 & 0.48 & -0.48 & 24 & $\ldots$ & $\ldots$ & -0.83 & $\ldots$ \\
\hline Sm II & 4642.23 & 0.38 & -0.46 & 24 & $\ldots$ & $\ldots$ & -0.80 & $\ldots$ \\
\hline Sm II & 4669.64 & 0.28 & -0.53 & 24 & $\ldots$ & $\ldots$ & -0.86 & $\ldots$ \\
\hline Sm II & 4719.84 & 0.04 & -1.24 & 24 & $\ldots$ & $\ldots$ & -1.01 & $\ldots$ \\
\hline Sm II & 4815.80 & 0.18 & -0.82 & 24 & $\ldots$ & $\ldots$ & -0.95 & $\ldots$ \\
\hline Eu II & 3724.93 & 0.00 & -0.09 & 25 & -1.32 & $\ldots$ & -1.12 & $\ldots$ \\
\hline Eu II & 3819.67 & 0.00 & +0.51 & 25 & -1.38 & -2.85 & $\ldots$ & -2.00 \\
\hline Eu II & 3907.11 & 0.21 & +0.17 & 25 & -1.35 & $\ldots$ & -1.21 & -1.97 \\
\hline Eu II & 4129.72 & 0.00 & +0.22 & 25 & -1.43 & -2.77 & -1.24 & -2.00 \\
\hline Eu II & 4205.04 & 0.00 & +0.21 & 25 & -1.34 & -2.71 & $\ldots$ & -1.88 \\
\hline Gd II & 3549.36 & 0.24 & +0.29 & 26 & -0.80 & $\ldots$ & -0.69 & $\ldots$ \\
\hline Gd II & 3557.06 & 0.60 & +0.04 & 26 & $\ldots$ & $\ldots$ & $-0.47:$ & $\ldots$ \\
\hline Gd II & 3712.70 & 0.38 & +0.04 & 26 & $\ldots$ & $\ldots$ & -0.72 & $\ldots$ \\
\hline Gd II & 3768.40 & 0.08 & +0.21 & 26 & -0.83 & -2.30 & -0.66 & $\ldots$ \\
\hline Gd II & 4037.89 & 0.56 & -0.42 & 26 & -0.76 & $\ldots$ & -0.69 & $\ldots$ \\
\hline Gd II & 4049.85 & 0.99 & +0.49 & 26 & -0.92 & $\ldots$ & $\ldots$ & $\ldots$ \\
\hline Gd II & 4085.56 & 0.73 & +0.00 & 26 & $\ldots$ & $\ldots$ & -0.73 & $\ldots$ \\
\hline Gd II & 4130.37 & 0.73 & -0.02 & 27 & -0.64 & $\ldots$ & -0.51 & $\ldots$ \\
\hline Gd II & 4215.02 & 0.43 & -0.44 & 26 & -0.88 & $\ldots$ & -0.66 & $\ldots$ \\
\hline Gd II & 4251.73 & 0.38 & -0.22 & 26 & $\ldots$ & $\ldots$ & -0.78 & $\ldots$ \\
\hline Tb II & 3600.41 & 0.64 & +0.60 & 28 & -1.29 : & $\ldots$ & $\ldots$ & $\ldots$ \\
\hline Tb II & 3702.85 & 0.13 & +0.44 & 28 & $\ldots$ & $\ldots$ & -1.61 & $\ldots$ \\
\hline Dy II & 3531.71 & 0.00 & +0.77 & 29 & -0.77 & $\ldots$ & -0.48 & -1.43 \\
\hline Dy II & 3536.02 & 0.54 & +0.53 & 29 & -0.74 & $\ldots$ & -0.51 & $\ldots$ \\
\hline Dy II & 3550.22 & 0.59 & +0.27 & 29 & -0.56 & $\ldots$ & $\ldots$ & -1.19 \\
\hline Dy II & 3563.15 & 0.10 & -0.36 & 29 & -0.74 & $\ldots$ & -0.57 & $\ldots$ \\
\hline Dy II & 3694.81 & 0.10 & -0.11 & 29 & -0.77 & -2.39 & -0.66 & -1.36 \\
\hline Dy II & 3757.37 & 0.10 & -0.17 & 29 & -0.77 & $\ldots$ & $\ldots$ & $\ldots$ \\
\hline
\end{tabular}


Table 3

(Continued)

\begin{tabular}{|c|c|c|c|c|c|c|c|c|}
\hline Species & $\begin{array}{c}\lambda \\
(\AA)\end{array}$ & $\begin{array}{l}\mathrm{EP} \\
(\mathrm{eV})\end{array}$ & $\log (g f)$ & Ref. & $\begin{array}{c}\log \epsilon \\
\text { HD } 108317\end{array}$ & $\begin{array}{c}\log \epsilon \\
\text { HD } 122563\end{array}$ & $\begin{array}{c}\log \epsilon \\
\text { HD } 126238\end{array}$ & $\begin{array}{c}\log \epsilon \\
\text { HD } 128279\end{array}$ \\
\hline Dy II & 3944.68 & 0.00 & +0.11 & 29 & -0.80 & $\ldots$ & $\ldots$ & -1.39 \\
\hline Dy II & 3996.69 & 0.59 & -0.26 & 29 & -0.74 & $\ldots$ & -0.60 & $\ldots$ \\
\hline Dy II & 4073.12 & 0.54 & -0.32 & 29 & -0.85 & $\ldots$ & -0.69 & $\ldots$ \\
\hline Dy II & 4103.31 & 0.10 & -0.38 & 29 & $\ldots$ & $\ldots$ & -0.57 & $\ldots$ \\
\hline Dy II & 4449.70 & 0.00 & -1.03 & 29 & $\ldots$ & $\ldots$ & -0.69 & $\ldots$ \\
\hline Ho II & 3456.01 & 0.00 & +0.76 & 30 & $-1.47:$ & $<-2.43$ & $-1.37:$ & $<-1.71$ \\
\hline Ho II & 3890.97 & 0.08 & +0.46 & 30 & -1.51 & $\ldots$ & $\ldots$ & $\ldots$ \\
\hline Er II & 3692.65 & 0.05 & +0.28 & 31 & -0.95 & $\ldots$ & $\ldots$ & $-1.48:$ \\
\hline Er II & 3729.52 & 0.00 & -0.59 & 31 & -0.89 & $\ldots$ & -0.78 & $\ldots$ \\
\hline Er II & 3786.84 & 0.00 & -0.52 & 31 & -0.92 & $\ldots$ & $\ldots$ & $\ldots$ \\
\hline Er II & 3830.48 & 0.00 & -0.22 & 31 & $\ldots$ & $\ldots$ & $\ldots$ & -1.63 \\
\hline Er II & 3896.23 & 0.05 & -0.12 & 31 & -0.92 & $\ldots$ & $\ldots$ & -1.48 \\
\hline Er II & 3906.31 & 0.00 & +0.12 & 31 & -1.01 & $\ldots$ & $\ldots$ & $\ldots$ \\
\hline Tm II & 3701.36 & 0.00 & -0.54 & 32 & $\ldots$ & $\ldots$ & -1.54 & $\ldots$ \\
\hline Tm II & 3795.76 & 0.03 & -0.23 & 32 & -1.86 & $\ldots$ & -1.66 & $\ldots$ \\
\hline Tm II & 3848.02 & 0.00 & -0.14 & 32 & -1.74 & $\ldots$ & -1.60 & -2.24 \\
\hline $\mathrm{Yb}$ II & 3694.19 & 0.00 & -0.30 & 33 & -1.13 & -2.69 & -0.93 & -1.69 \\
\hline Lu II & 2615.41 & 0.00 & +0.11 & 34 & -1.64 & -2.89 & $\ldots$ & -1.64 \\
\hline Hf II & 2641.41 & 1.04 & +0.57 & 35 & -0.87 & $-2.47:$ & -0.78 & -1.07 \\
\hline Hf II & 3176.85 & 0.61 & -0.80 & 35 & $\ldots$ & $<-1.13$ & $\ldots$ & $\ldots$ \\
\hline Hf II & 4093.15 & 0.45 & -1.15 & 35 & -1.16 & $\ldots$ & -1.09 & $\ldots$ \\
\hline Os I & 3058.66 & 0.00 & -0.41 & 36 & -0.50 & $<-1.25$ & -0.38 : & $<-0.39$ \\
\hline Os II & 2282.28 & 0.00 & -0.05 & 37 & -0.88 & $\ldots$ & -0.86 & $<-1.19$ \\
\hline Ir I & 2924.79 & 0.00 & -0.66 & 38 & $-0.49:$ & $<-1.35$ & $-0.34:$ & $<-0.29$ \\
\hline Ir I & 3220.78 & 0.35 & -0.48 & 38 & +0.08 & $<-0.73$ & $\ldots$ & $\ldots$ \\
\hline Ir I & 3800.12 & 0.00 & -1.43 & 38 & -0.10 & $<-0.42$ & -0.26 & $<+0.01$ \\
\hline Pt I & 2659.45 & 0.00 & -0.03 & 39 & -0.49 & $<-2.15$ & $\ldots$ & $<-1.19$ \\
\hline Pt I & 2929.79 & 0.00 & -0.70 & 39 & -0.43 & $<-1.25$ & $-0.34:$ & $<-0.49$ \\
\hline $\mathrm{Au} I$ & 2675.95 & 0.00 & -0.47 & 40 & $-1.64:$ & $\ldots$ & $\ldots$ & $<-0.49$ \\
\hline $\mathrm{Pb}$ I & 2833.05 & 0.00 & -0.50 & 41 & $<-0.26$ & $<-1.45$ & $<+0.03$ & $<-0.09$ \\
\hline $\mathrm{PbI}$ & 3683.46 & 0.97 & -0.54 & 41 & $<+0.35$ & $<-0.32$ & -0.02 & $\ldots$ \\
\hline $\mathrm{Pb}$ I & 4057.81 & 1.32 & -0.22 & 41 & $<+0.25$ & $<-0.65$ & +0.31 & $<+0.35$ \\
\hline Bi I & 3024.64 & 1.91 & -0.14 & 4 & $<+1.04$ & $<+0.15$ & $<+1.43$ & $<+0.91$ \\
\hline Th II & 4019.13 & 0.00 & -0.23 & 42 & -1.99 & $\ldots$ & $\ldots$ & $\ldots$ \\
\hline
\end{tabular}

Notes. The $\log \epsilon$ abundances listed in the table have been corrected according to the values given in Table 10.

${ }^{a}$ This feature is composed of two Pr II transitions, the $\lambda 4429.13$ transition with $\mathrm{EP}=0.00 \mathrm{eV}$ and $\log (g f)=-0.49$ and the $\lambda 4429.26$ transition with $\mathrm{EP}=0.37 \mathrm{eV}$ and $\log (g f)=-0.05$.

References. (1) Fuhr \& Wiese 2009, using hfs/IS from J. S. Sobeck et al. 2012, in preparation; (2) Fuhr \& Wiese 2009, using hfs/IS from Kurucz \& Bell 1995; (3) Roederer \& Lawler 2012; (4) Fuhr \& Wiese 2009; (5) Migdalek \& Baylis 1987; (6) Biémont et al. 2011; (7) Ljung et al. 2006; (8) Malcheva et al. 2006; (9) Nilsson \& Ivarsson 2008, using hfs from Nilsson et al. 2010 when available; (10) Whaling \& Brault 1988; (11) Sikström et al. 2001; (12) Wickliffe et al. 1994; (13) Duquette \& Lawler 1985; (14) Kwiatkowski et al. 1982; (15) Xu et al. 2006; (16) Hansen et al. 2012 for both $\log (g f)$ and hfs/IS; (17) Morton 2000; (18) Roederer et al. 2012; (19) Fuhr \& Wiese 2009, using hfs/IS from McWilliam 1998; (20) Lawler et al. 2001a, using hfs from Ivans et al. 2006 when available; (21) Lawler et al. 2009; (22) Li et al. 2007, using hfs from Sneden et al. 2009; (23) Den Hartog et al. 2003, using hfs/IS from Roederer et al. 2008 when available; (24) Lawler et al. 2006, using hfs/IS from Roederer et al. 2008 when available; (25) Lawler et al. 2001c, using hfs/IS from Ivans et al. 2006; (26) Den Hartog et al. 2006; (27) previously unpublished (uncertainty of 25\%); (28) Lawler et al. 2001b, using hfs from Lawler et al. 2009; (29) Wickliffe et al. 2000; (30) Lawler et al. 2004 for both $\log (g f)$ and hfs; (31) Lawler et al. 2008; (32) Wickliffe \& Lawler 1997; (33) Sneden et al. 2009 for both $\log (g f)$ and hfs/IS; (34) Roederer et al. 2010b, using hfs presented in Appendix B; (35) Lawler et al. 2007; (36) Quinet et al. 2006, which is only 0.01 dex different from Cowan et al. 2005 for this line; (37) Quinet et al. 2006; (38) Xu et al. 2007, using hfs/IS from Cowan et al. 2005 when available; (39) Den Hartog et al. 2005 for both $\log (g f)$ and hfs/IS; (40) Fivet et al. 2006; (41) Biémont et al. 2000, using hfs presented in Appendix C; (42) Nilsson et al. 2002.

our spectra. Their EWs differ by a factor of two within the uncertainties $(70 \pm 1 \mathrm{~m} \AA$ and $34 \pm 1 \mathrm{~m} \AA)$, which matches the ratio of their $f$-values and suggests that neither line is saturated. We calculate column densities $\log N(\mathrm{NaI})=11.76 \pm 0.01 \mathrm{~cm}^{-2}$ toward HD 126238 and $\log N(\mathrm{Na} \mathrm{I})=11.55 \pm 0.01 \mathrm{~cm}^{-2}$ toward HD 128279. Using the linear relationship between $\log N(\mathrm{NaI})$ and $\log N\left(\mathrm{HI}+\mathrm{H}_{2}\right)=\log N(\mathrm{H})$ found by Ferlet et al. (1985), we assume a common $\mathrm{Na} / \mathrm{H}$ ratio and $\mathrm{Na}$ depletion in the solar neighborhood to estimate $\log N(\mathrm{H})=20.05 \mathrm{~cm}^{-2}$ toward
HD 126238 and $\log N(\mathrm{H})=19.85 \mathrm{~cm}^{-2}$ toward HD 128279 . Upper limits from the non-detection of interstellar Na I toward HD 108317 and HD 122563 imply $\log N(\mathrm{NaI})<10.71 \mathrm{~cm}^{-2}$ and $\log N(\mathrm{H})<19.04 \mathrm{~cm}^{-2}$.

Using the mean relationship between $N\left(\mathrm{HI}+\mathrm{H}_{2}\right)$ and $E(B-V)$ derived by Bohlin et al. (1978), we would infer $E(B-V)$ between 0.01 and 0.02 toward HD 126238 and HD 128279 and even less toward HD 108317 and HD 122563. This is substantially less than the amount of reddening predicted 
Table 4

Iron Equivalent Widths

\begin{tabular}{|c|c|c|c|c|}
\hline $\begin{array}{l}\lambda \\
(\AA)\end{array}$ & $\begin{array}{l}\text { HD } 108317 \\
\text { EW (mA) }\end{array}$ & $\begin{array}{c}\text { HD } 122563 \\
\text { EW }(\mathrm{mA})\end{array}$ & $\begin{array}{l}\text { HD } 126238 \\
\text { EW (mA) }\end{array}$ & $\begin{array}{c}\text { HD } 128279 \\
\text { EW }(\mathrm{mA})\end{array}$ \\
\hline \multicolumn{5}{|c|}{$\mathrm{Fe}_{\mathrm{I}}$} \\
\hline 3765.54 & $\ldots$ & $\ldots$ & 117.1 & $\ldots$ \\
\hline
\end{tabular}

(This table is available in its entirety in a machine-readable form in the online journal. A portion is shown here for guidance regarding its form and content.)

by the Schlegel et al. (1998) dust maps. We would only expect $\approx 40-60 \mathrm{~K}$ of a decrease in $T_{\text {eff }}$ based on these values, which is far short of the adjustments found necessary to produce no correlation between iron abundance and EP. We note, however, that there is more than a factor of five in the scatter between $\log N(\mathrm{NaI})$ and $\log N(\mathrm{H})$ at these column densities, and the relationship between $N\left(\mathrm{HI}+\mathrm{H}_{2}\right)$ and $E(B-V)$ is poorly defined at low reddening. The actual amount of gas along the lines of sight to HD 126238 and HD 128279 could be higher by a factor of a few, which would bring the $T_{\text {eff }}$ adjustments into line with those found for the unreddened stars HD 108317 and HD 122563.

Other comparisons of the spectra suggest that this explanation is plausible. Figure 8 illustrates the profiles of the $\mathrm{H} \alpha$ line in all four stars. The line is broadest and virtually indistinguishable in HD 108317 and HD 128279. HD 126238 and HD 122563 have narrower profiles. Our derived model parameters for HD 108317 and HD 128279 are identical within the uncertainties, and Figures 1-7 demonstrate how similar the spectra of these two stars are. This confirms the relative sense of our spectroscopic temperatures, and it supports our decision to adjust the temperatures from the initial photometric estimates to a spectroscopic scale.

\section{ABUNDANCE ANALYSIS}

We perform the abundance analysis by comparing the observed spectrum with synthetic spectra calculated using MOOG. Sources for the atomic data for lines of interest are presented in Table 3. We use damping constants from Barklem et al. (2000) and Barklem \& Aspelund-Johansson (2005), when available, and otherwise we resort to the standard Unsöld (1955) approximation. We adopt the SS isotopic fractions for $\mathrm{Cu}$ I and the $r$-process isotopic fractions reported in Sneden et al. (2008) for all other elements where multiple isotopes are considered in the calculation (Ba II, Nd II, Sm II, Eu II, Yb II, Ir I, Pt I, and Pb I).

When our lines of interest are mildly blended with other absorption features for which no laboratory $\log (g f)$ value is available, we treat the strength of the blending feature as a free parameter. When the strength of blending features is completely degenerate with the strength (abundance) of lines of interest, we discard these lines from consideration. Some unidentified absorption features are detected in our stellar spectra but not present in the line lists, and many lines in the UV lack reliable transition probabilities. This is a well-known problem in the UV (e.g., Leckrone et al. 1999). When no transition in our line list can be reasonably adjusted to account for this absorption, we assume that the absorption is due to an uncatalogued Fe I line with a lower excitation level of $1.5 \mathrm{eV}$ and treat the $\log (g f)$ value as a free parameter in our synthesis. This technique has been successfully applied previously by, e.g., Peterson (2011).

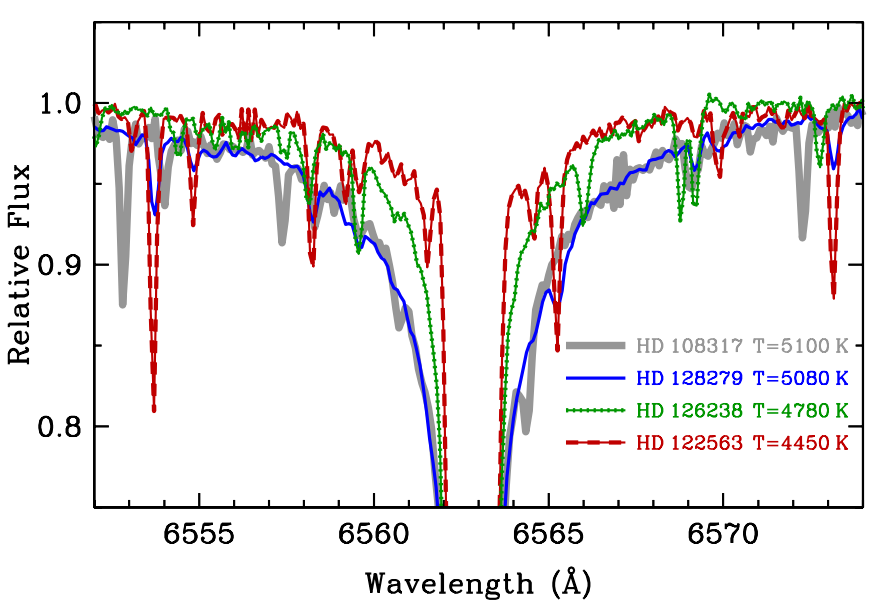

Figure 8. Comparison of the $\mathrm{H} \alpha$ line profiles for the four program stars. For reference we list the temperatures derived by minimizing the $\mathrm{Fe}$ I abundance correlation with EP. The two warmest stars, HD 108317 and HD 128279, have nearly identical $\mathrm{H} \alpha$ profiles. HD 126238 and HD 122563 are cooler, and the $\mathrm{H} \alpha$ line profiles confirm the spectroscopic temperatures in a relative sense.

(A color version of this figure is available in the online journal.)

Our HIRES and MIKE spectra overlap in wavelength coverage. We find no significant difference between abundances derived from the HIRES and MIKE spectra for these stars. When a line is present in both, we adopt the abundance derived from the higher resolution HIRES spectrum.

Tables 7 and 8 present the absolute and relative abundances, statistical and total uncertainties, and the number of lines examined in each star. The reference solar abundances listed in these tables are taken from Asplund et al. (2009). Mean abundances are weighted by the statistical uncertainties of each line. The statistical uncertainties quoted in these tables include components from the synthetic spectra fitting, uncertainties in the individual $\log (g f)$ values, and uncertainties from the wavelength-dependent corrections discussed in Section 8. We assume that the minimum uncertainty per line is equivalent to the standard deviation of the well-measured species $\mathrm{Zr}$ II for each star (0.11-0.17 dex). Lines whose fit is poorer due to significant blending or uncertainty in the continuum identification are marked with a colon in Table 3 and given lower weight in the average. Systematic uncertainties for each of neutral and singly ionized species have been discussed in more detail by Cowan et al. (2005), and we adopt their assessment of the uncertainties. These uncertainties amount to 0.12 and 0.17 dex for neutral and ionized species, respectively, and are added in quadrature with the statistical uncertainties to form the total uncertainties listed in Tables 7 and 8.

The dominant sources of continuous opacity in these stars at the wavelengths considered are $\mathrm{H}^{-}$bound-free absorption and Rayleigh scattering from neutral H. In metal-rich stars, metal ionization makes significant contributions to the continuous opacity, but this is insignificant in the metal-poor stars considered here (see Roederer 2012a).

\section{ABUNDANCE TRENDS WITH WAVELENGTH}

We measure EWs of 119-134 Fe I and II lines, spanning $\lambda \lambda 3765-6750$, in each star in our sample (see Table 4). At the referee's suggestion, we also examine Fe I and II lines at wavelengths as short as $\lambda 2283$. We derive the abundances of all iron lines not listed in Table 4 by spectrum synthesis since these additional lines lie in more crowded spectral regions. The final 
Table 5

Basic Data and Initial $T_{\text {eff }}$ and $\log g$ Estimates

\begin{tabular}{lcccccccc}
\hline \hline Star & $\begin{array}{c}\pi \\
(\mathrm{mas})\end{array}$ & $\begin{array}{c}D \\
(\mathrm{pc})\end{array}$ & $V$ & $K$ & $E(B-V)$ & $(V-K)_{0}$ & $\begin{array}{c}T_{\text {eff }}(V-K) \\
(\mathrm{K})\end{array}$ & $\log g(\pi)$ \\
\hline HD 108317 & $5.73 \pm 0.67$ & $175 \pm 21$ & 8.05 & 6.153 & 0.018 & 1.849 & $5310 \pm 75$ & $2.90 \pm 0.16$ \\
HD 122563 & $4.22 \pm 0.35$ & $237 \pm 20$ & 6.20 & 3.731 & 0.025 & 2.386 & $4680 \pm 65$ & $1.58 \pm 0.13$ \\
HD 126238 & $3.82 \pm 0.77$ & $262 \pm 55$ & 7.66 & 5.338 & 0.115 & 2.003 & $5100 \pm 72$ & $2.09 \pm 0.26$ \\
HD 128279 & $6.09 \pm 1.08$ & $164 \pm 30$ & 8.02 & 6.065 & 0.100 & 1.681 & $5550 \pm 79$ & $2.85 \pm 0.23$ \\
\hline
\end{tabular}

Table 6

Adopted Model Parameters and Derived Metallicities

\begin{tabular}{|c|c|c|c|c|c|c|c|c|}
\hline Star & $\begin{array}{l}T_{\text {eff }} \\
(\mathrm{K})\end{array}$ & $\log g$ & $\begin{array}{c}v_{t} \\
\left(\mathrm{~km} \mathrm{~s}^{-1}\right)\end{array}$ & {$[\mathrm{M} / \mathrm{H}]$} & {$[\mathrm{Fe} \mathrm{I} / \mathrm{H}]$} & $N$ & {$[\mathrm{Fe}$ II $/ \mathrm{H}]$} & $N$ \\
\hline HD 108317 & 5100 & 2.67 & 1.50 & -2.37 & $-2.53 \pm 0.09$ & 300 & $-2.37 \pm 0.14$ & 55 \\
\hline HD 122563 & 4450 & 1.37 & 2.00 & -2.61 & $-3.06 \pm 0.10$ & 211 & $-2.61 \pm 0.12$ & 20 \\
\hline HD 126238 & 4780 & 1.72 & 1.60 & -1.93 & $-1.98 \pm 0.10$ & 217 & $-1.93 \pm 0.10$ & 26 \\
\hline HD 128279 & 5080 & 2.57 & 1.60 & -2.46 & $-2.48 \pm 0.09$ & 266 & $-2.46 \pm 0.14$ & 48 \\
\hline
\end{tabular}

Notes. The $[\mathrm{Fe} / \mathrm{H}]$ values presented in this table are derived from individual line abundances that have been corrected according to the values presented in Table 10 .

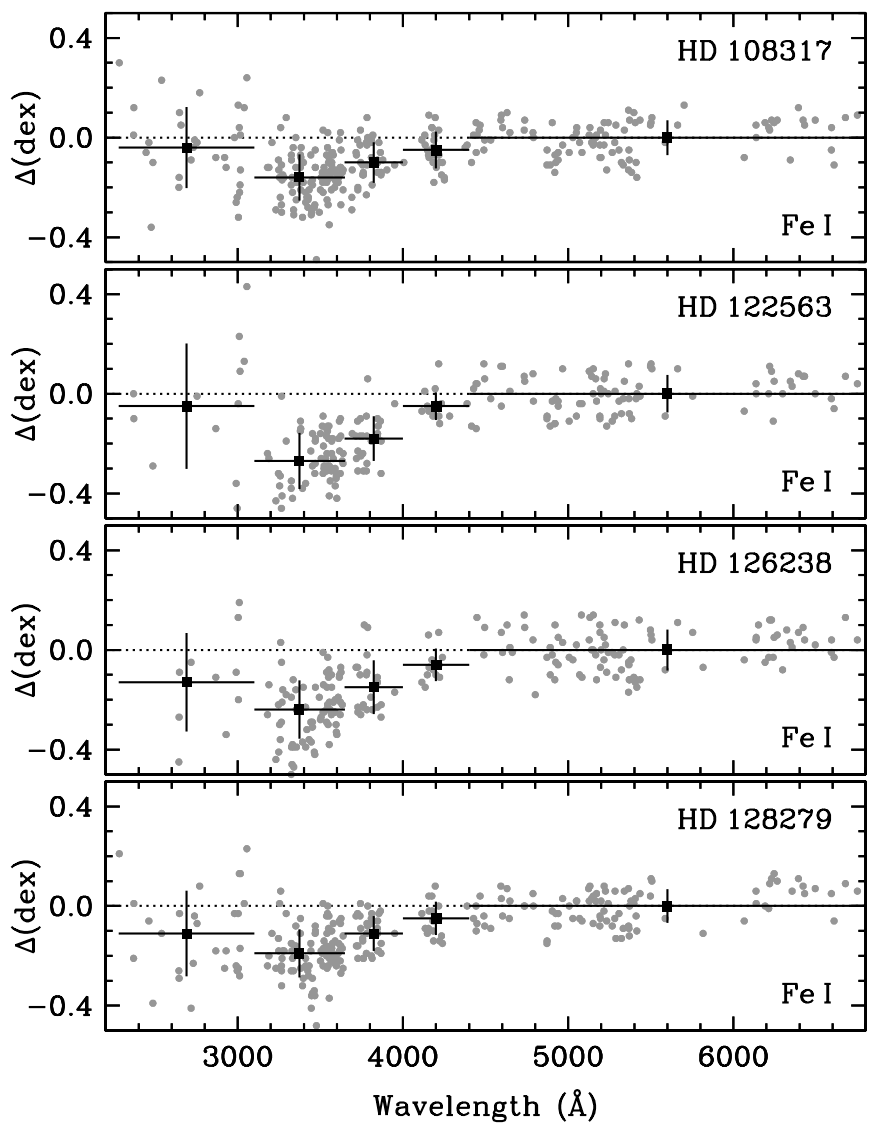

Figure 9. Derived iron abundances from $\mathrm{Fe}_{\mathrm{I}}$ lines as a function of wavelength The gray dots represent abundances derived from individual lines. Large black squares represent the mean abundance in each wavelength interval. Vertical error bars indicate the standard deviation, and horizontal error bars mark the wavelength interval. The zero point for each star is defined as the iron abundance derived from $\mathrm{Fe}$ I lines longward of $\lambda 4400$, which is marked by the dotted line in each panel.

list of iron abundances is presented in Table 9, which is available only in the online version of the journal. A sample is shown in the printed edition to demonstrate its form and content.

Figure 9 illustrates the iron abundances derived from Fe I, by far the species with the most lines considered (211-300 lines

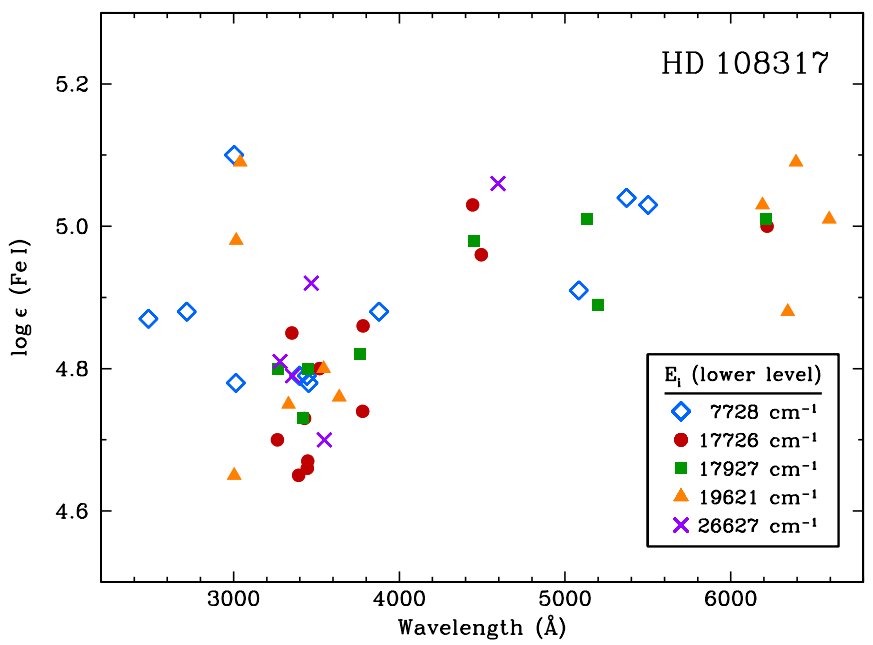

Figure 10. Derived iron abundances from Fe I lines in HD 108317 as a function of wavelength for select lower levels.

(A color version of this figure is available in the online journal.)

per star). Significant variations in Fe I persist at short wavelengths. These variations are characterized as a decrease in the average abundance derived from $\mathrm{Fe}$ I lines at short wavelengths compared to lines at long wavelengths. When compared with abundances derived from $\mathrm{Fe}$ I lines at wavelengths longward of $\lambda 4400$, Fe I lines short of $\approx \lambda 3100$ and between $\lambda \lambda 4000$ and 4400 yield average abundances lower by small amounts, typically $0.04-0.06 \mathrm{dex}$, but as large as $0.13 \mathrm{dex}$. Iron abundances derived from $\mathrm{Fe}$ I lines with wavelengths between $\approx 3100$ and $\approx \lambda 4000$ show an even larger deficiency of $0.10-0.27$ dex.

All four stars qualitatively show the same effect, although the magnitude of the "dip" appears to be larger in the cooler stars. Our tests with warmer metal-poor subgiant stars suggest that they, too, exhibit a qualitatively similar feature at these wavelengths. Omitting lines with the largest transition probability uncertainties does not change the result appreciably.

If departures from LTE in neutral iron are the source of this effect, we might expect lines originating from different lower levels to behave differently. Figure 10 demonstrates that this is not the case for HD 108317, the warmest star in our sample, 
Table 7

Derived Final Abundances I

\begin{tabular}{|c|c|c|c|c|c|c|c|c|c|c|c|c|}
\hline \multirow[t]{2}{*}{ Species } & \multirow[t]{2}{*}{$Z$} & \multirow{2}{*}{$\begin{array}{c}\text { SS } \\
\log \epsilon \\
\end{array}$} & \multicolumn{5}{|c|}{ HD 108317} & \multicolumn{5}{|c|}{ HD 122563} \\
\hline & & & $\log \epsilon$ & {$[\mathrm{X} / \mathrm{Fe}]$} & $\sigma_{\text {stat }}$ & $\sigma_{\text {tot }}$ & $N$ & $\log \epsilon$ & {$[\mathrm{X} / \mathrm{Fe}]$} & $\sigma_{\text {stat }}$ & $\sigma_{\mathrm{tot}}$ & $N$ \\
\hline $\mathrm{Fe} \mathrm{I}$ & 26 & +7.50 & +4.97 & +0.00 & 0.09 & 0.15 & 300 & +4.44 & +0.00 & 0.10 & 0.16 & 211 \\
\hline Fe II & 26 & +7.50 & +5.13 & +0.00 & 0.14 & 0.22 & 55 & +4.89 & +0.00 & 0.12 & 0.20 & 20 \\
\hline $\mathrm{Cu} \mathrm{I}$ & 29 & +4.19 & +0.70 & -0.96 & 0.11 & 0.16 & 2 & +0.10 & -1.03 & 0.18 & 0.22 & 1 \\
\hline $\mathrm{Zn} I$ & 30 & +4.56 & +2.26 & +0.23 & 0.09 & 0.15 & 3 & +1.99 & +0.49 & 0.17 & 0.21 & 4 \\
\hline Ge I & 32 & +3.65 & +0.17 & -0.95 & 0.12 & 0.17 & 3 & -0.88 & -1.47 & 0.17 & 0.21 & 3 \\
\hline $\mathrm{Rb} I$ & 37 & +2.52 & $<+1.40$ & $<+1.41$ & $\ldots$ & $\ldots$ & 1 & $<+1.20$ & $<+1.74$ & $\ldots$ & $\ldots$ & 1 \\
\hline Sr II & 38 & +2.87 & +0.48 & -0.02 & 0.10 & 0.19 & 2 & -0.02 & -0.28 & 0.09 & 0.19 & 3 \\
\hline$Y_{\text {II }}$ & 39 & +2.21 & -0.42 & -0.26 & 0.11 & 0.20 & 10 & -0.95 & -0.55 & 0.13 & 0.21 & 10 \\
\hline Zr II & 40 & +2.58 & +0.45 & +0.24 & 0.11 & 0.20 & 39 & -0.16 & -0.13 & 0.14 & 0.22 & 26 \\
\hline $\mathrm{Nb}$ II & 41 & +1.46 & -0.86 & +0.05 & 0.10 & 0.19 & 3 & -1.52 & -0.37 & 0.14 & 0.22 & 3 \\
\hline Mo I & 42 & +1.88 & -0.23 & +0.42 & 0.14 & 0.19 & 1 & -1.22 & -0.04 & 0.29 & 0.31 & 1 \\
\hline Mo II & 42 & +1.88 & $<+0.24$ & $<+0.73$ & $\ldots$ & $\ldots$ & 1 & $<-0.65$ & $<+0.08$ & $\ldots$ & $\ldots$ & 1 \\
\hline $\mathrm{Ru} \mathrm{I}_{\mathrm{I}}$ & 44 & +1.75 & -0.04 & +0.74 & 0.11 & 0.16 & 2 & -1.04 & +0.27 & 0.24 & 0.27 & 3 \\
\hline $\mathrm{Rh} I$ & 45 & +1.06 & -0.84 & +0.63 & 0.24 & 0.27 & 1 & -1.73 & +0.27 & 0.29 & 0.31 & 1 \\
\hline Pd I & 46 & +1.65 & -0.67 & +0.21 & 0.13 & 0.18 & 2 & -1.78 & -0.37 & 0.30 & 0.32 & 1 \\
\hline $\mathrm{Ag}_{\mathrm{I}}$ & 47 & +1.20 & -1.34 & -0.01 & 0.17 & 0.21 & 2 & $<-1.63$ & $<+0.23$ & $\ldots$ & $\ldots$ & 2 \\
\hline $\mathrm{Cd}_{\mathrm{I}}$ & 48 & +1.71 & -0.87 & -0.05 & 0.25 & 0.28 & 1 & $<-2.85$ & $<-1.50$ & $\ldots$ & $\ldots$ & 1 \\
\hline Sn I & 50 & +2.07 & $<+0.80$ & $<+1.26$ & $\ldots$ & $\ldots$ & 1 & $<+0.38$ & $<+1.37$ & $\ldots$ & $\ldots$ & 1 \\
\hline $\mathrm{Te}_{\mathrm{I}}$ & 52 & +2.18 & +0.02 & +0.37 & 0.21 & 0.24 & 1 & $\ldots$ & $\ldots$ & $\ldots$ & $\ldots$ & . \\
\hline Ba II & 56 & +2.18 & -0.32 & -0.13 & 0.10 & 0.19 & 4 & -1.78 & -1.35 & 0.14 & 0.22 & 2 \\
\hline La II & 57 & +1.10 & -1.12 & +0.15 & 0.05 & 0.17 & 7 & -2.48 & -0.97 & 0.18 & 0.24 & 3 \\
\hline Ce II & 58 & +1.58 & -0.68 & +0.11 & 0.04 & 0.17 & 11 & -1.94 & -0.91 & 0.07 & 0.18 & 6 \\
\hline Pr II & 59 & +0.72 & -0.90 & +0.75 & 0.16 & 0.23 & 2 & $<-2.15$ & $<-0.26$ & $\ldots$ & $\ldots$ & 1 \\
\hline Nd II & 60 & +1.42 & -0.71 & +0.24 & 0.07 & 0.18 & 12 & -2.06 & -0.87 & 0.09 & 0.19 & 3 \\
\hline Sm II & 62 & +0.96 & -1.05 & +0.36 & 0.11 & 0.20 & 5 & -2.31 & -0.66 & 0.11 & 0.20 & 3 \\
\hline Eu II & 63 & +0.52 & -1.37 & +0.48 & 0.06 & 0.18 & 5 & -2.77 & -0.68 & 0.09 & 0.19 & 3 \\
\hline Gd II & 64 & +1.07 & -0.82 & +0.48 & 0.10 & 0.19 & 6 & -2.30 & -0.76 & 0.17 & 0.24 & 1 \\
\hline Tb II & 65 & +0.30 & -1.30 & +0.77 & 0.24 & 0.29 & 1 & $\ldots$ & $\ldots$ & $\ldots$ & $\ldots$ & $\ldots$ \\
\hline Dy II & 66 & +1.10 & -0.75 & +0.52 & 0.08 & 0.18 & 9 & -2.39 & -0.88 & 0.17 & 0.24 & 1 \\
\hline Ho II & 67 & +0.48 & -1.50 & +0.39 & 0.12 & 0.20 & 2 & $<-2.43$ & $<-0.30$ & $\ldots$ & $\ldots$ & 1 \\
\hline Er II & 68 & +0.92 & -0.94 & +0.51 & 0.06 & 0.18 & 5 & $\ldots$ & $\ldots$ & $\ldots$ & $\ldots$ & $\ldots$ \\
\hline Tm II & 69 & +0.10 & -1.80 & +0.47 & 0.10 & 0.19 & 2 & $\ldots$ & $\ldots$ & $\ldots$ & $\ldots$ & $\ldots$ \\
\hline Yb II & 70 & +0.92 & -1.13 & +0.32 & 0.14 & 0.22 & 1 & -2.69 & -1.00 & 0.17 & 0.24 & 1 \\
\hline Lu II & 71 & +0.10 & -1.64 & +0.63 & 0.20 & 0.26 & 1 & -2.89 & -0.38 & 0.29 & 0.33 & 1 \\
\hline Hf II & 72 & +0.85 & -0.99 & +0.53 & 0.21 & 0.27 & 2 & -2.47 & -0.71 & 0.38 & 0.41 & 1 \\
\hline Os I & 76 & +1.40 & -0.50 & +0.63 & 0.20 & 0.23 & 1 & $<-1.25$ & $<+0.41$ & $\ldots$ & $\ldots$ & 1 \\
\hline Os II & 76 & +1.40 & -0.88 & +0.09 & 0.20 & 0.26 & 1 & $\ldots$ & $\ldots$ & $\ldots$ & $\ldots$ & . \\
\hline Ir I & 77 & +1.38 & -0.07 & +1.08 & 0.32 & 0.34 & 3 & $<-1.35$ & $<+0.33$ & $\ldots$ & $\ldots$ & 3 \\
\hline $\mathrm{Pt} I$ & 78 & +1.62 & -0.46 & +0.45 & 0.14 & 0.19 & 2 & $<-2.15$ & $<-0.71$ & $\ldots$ & $\ldots$ & 2 \\
\hline $\mathrm{Au} \mathrm{I}$ & 79 & +0.80 & -1.64 & +0.09 & 0.27 & 0.30 & 1 & $\ldots$ & $\ldots$ & $\ldots$ & $\ldots$ & .. \\
\hline $\mathrm{Pb} \mathrm{I}$ & 82 & +2.04 & $<-0.26$ & $<+0.23$ & $\ldots$ & $\ldots$ & 3 & $<-1.45$ & $<-0.43$ & $\ldots$ & $\ldots$ & 3 \\
\hline Bi I & 83 & +0.65 & $<+1.04$ & $<+2.92$ & $\ldots$ & $\ldots$ & 1 & $<+0.15$ & $<+2.56$ & $\ldots$ & $\ldots$ & 1 \\
\hline Th II & 90 & +0.06 & -1.99 & +0.32 & 0.13 & 0.21 & 1 & $\ldots$ & $\ldots$ & $\ldots$ & $\ldots$ & $\ldots$ \\
\hline
\end{tabular}

and the results are similar for HD 122563, the coolest star in our sample. Figure 10 shows the iron abundance derived from Fe I lines as a function of wavelength for five selected lower levels. These levels are chosen to have a reasonably large set of high-quality $\log (g f)$ values both within and outside the affected wavelength region. The levels range from 0.96 to $3.3 \mathrm{eV}$, and all show the same effect of producing low iron abundances between $\lambda \lambda 3100$ and 4000 . This indicates that we are not observing a non-LTE distribution of level populations masquerading as a wavelength-dependent trend.

An underestimate of the continuous opacity in the region of the dip would lead to spuriously low abundance results relative to shorter and longer wavelengths. This would also affect abundances derived from lines of all species, not just Fe I, and we see hints of this in the abundances derived from, e.g., Fe II or $\mathrm{Zr}$ II lines.
The wavelengths of the dip hint that such an effect could be related to the transition from the Paschen continuum to the Balmer continuum. In an effort to test this as a possibility for wavelengths longer than the Balmer jump at $\lambda 3647$, we have modified MOOG to compute the H I bound-free continuous absorption according to the occupation probability formalism articulated by Däppen et al. (1987), Hummer \& Mihalas (1988), and Hubeny et al. (1994). This approximates the probability that a particular level is so strongly perturbed by interactions with other particles that an electron excited to this level is effectively unbound, or dissolved. This produces a continuous, density-dependent distribution from bound-bound transitions to bound-free in a pseudocontinuum for progressively higher levels in a given H I spectral series. This ensures that ionization occurs gradually rather than in sudden, discrete jumps at the series ionization edges. Our implementation of these new 
Table 8

Derived Final Abundances II

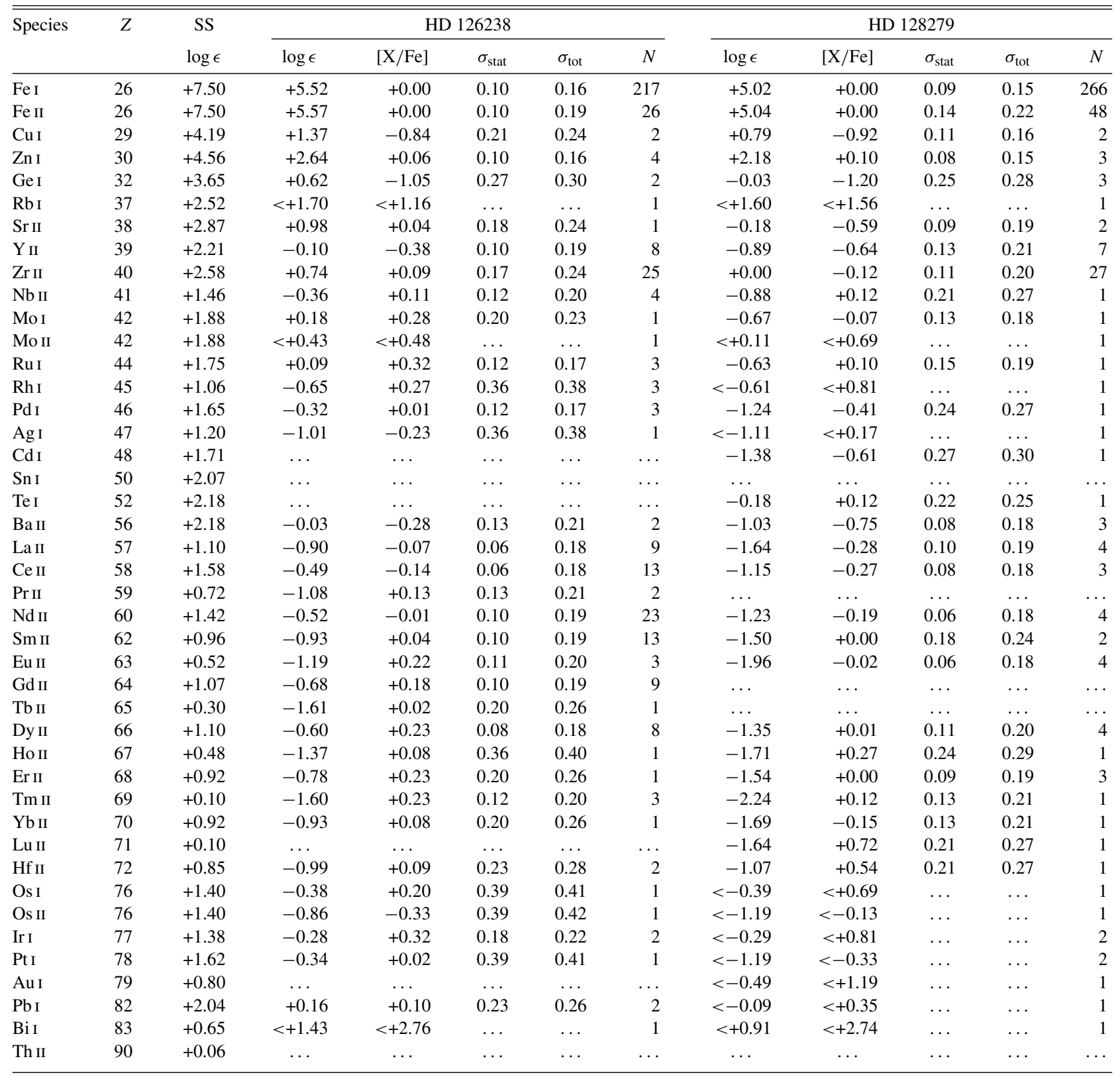

Table 9

Line-by-line Iron Abundances

\begin{tabular}{|c|c|c|c|c|c|c|}
\hline $\begin{array}{l}\text { Wavelength } \\
(\AA)\end{array}$ & $\begin{array}{c}\text { EP } \\
(\mathrm{eV})\end{array}$ & $\log (g f)$ & $\begin{array}{c}\text { HD } 108317 \\
\log \epsilon\end{array}$ & $\begin{array}{c}\text { HD } 122563 \\
\log \epsilon\end{array}$ & $\begin{array}{c}\text { HD } 126238 \\
\log \epsilon\end{array}$ & $\begin{array}{c}\text { HD } 128279 \\
\log \epsilon\end{array}$ \\
\hline \multicolumn{7}{|c|}{$\mathrm{Fe}_{\mathrm{I}}$} \\
\hline 2283.30 & 0.12 & -2.22 & 5.31 & $\ldots$ & $\ldots$ & 5.34 \\
\hline
\end{tabular}

Notes. Wavelength-dependent corrections derived from Fe I have been applied to the $\log \epsilon$ entries in this table. The uncorrected log $\epsilon$ values may be obtained by subtracting the values listed in the final column of Table 10 . All $\log (g f)$ values have been adopted from the critical compilation of Fuhr \& Wiese (2006).

(This table is available in its entirety in a machine-readable form in the online journal. A portion is shown here for guidance regarding its form and content.)

calculations in MOOG follows that in Version 10.1 of turbospectrum (Alvarez \& Plez 1998).

The results of these calculations for HD 108317 are shown in Figure 11. The (blue) dashed line and (green) solid line represent the Hi dissolved opacity and bound-bound opacity, respectively. Computation of the $\mathrm{H}$ I bound-free opacity, shown by the (red) studded line, has not been altered from the standard MOOG use of the ATLAS polynomial approximations (Kurucz 


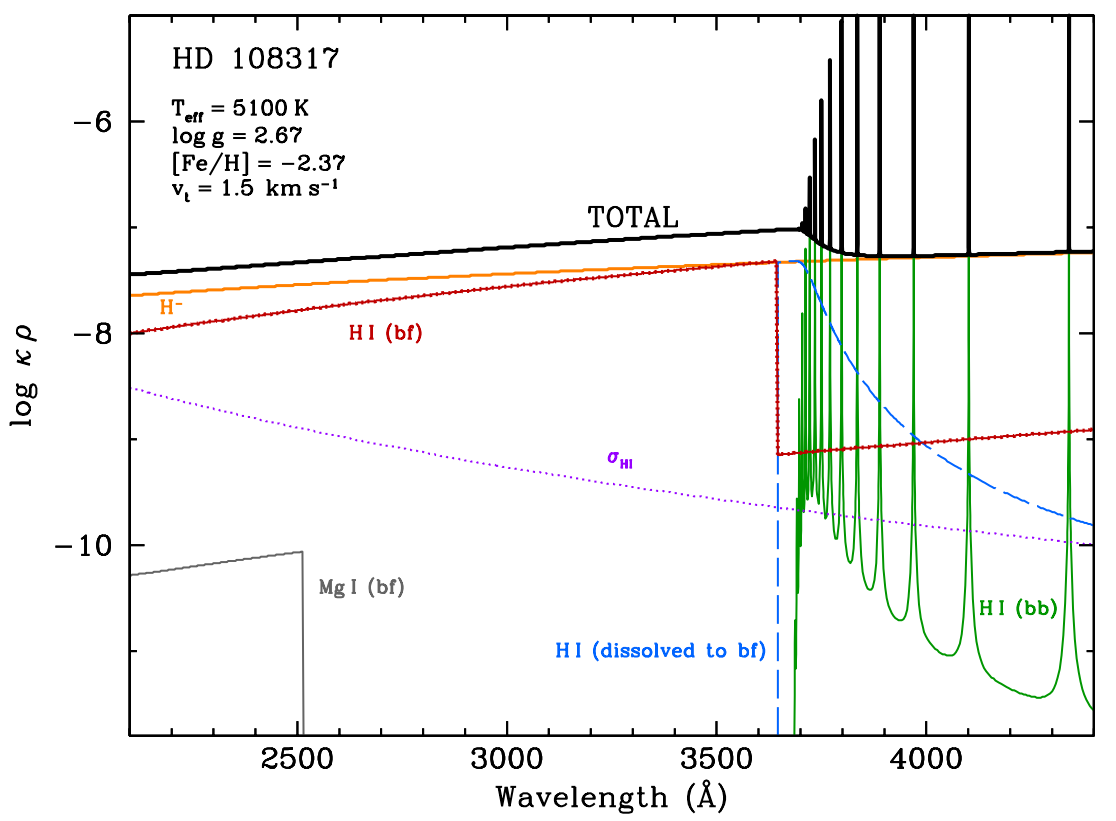

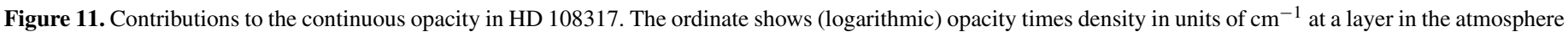

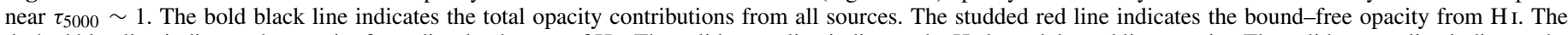

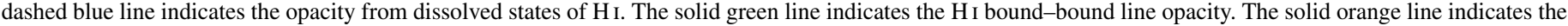

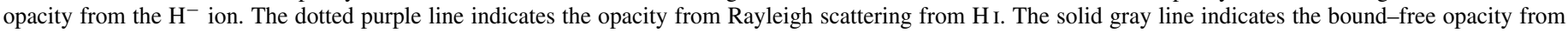
Mg I.

(A color version of this figure is available in the online journal.)

1970) to the Coulomb cross sections of Karzas \& Latter (1961). For this particular model atmosphere, the opacity contribution from the bound-free dissolved levels smoothly transitions into the bound-free absorption short of the $n=2$ series limit. In cooler models, however, the calculated bound-free opacity from dissolved levels is greater than the bound-free opacity short of the series limit, and the reverse is true for warmer models. In both cases, the discrepancy at the series limit is up to a factor of a few. While the increased opacity from the dissolved bound-free states does reduce the effect of the iron abundance dip, this does not completely eliminate the effect at wavelengths longward of the $n=2$ series limit. Furthermore, it cannot affect lines between $\lambda \lambda 3100$ and 3647 and only minimally affects lines longward of $\approx \lambda 3900$, where a small dip is still present.

We are unable to identify the source of the discrepancy among iron abundances derived from Fe I lines at blue wavelengths in our spectra. We have chosen to adopt an empirical, wavelengthdependent correction to the iron abundances, as shown by the black squares in Figure 9. These corrections are computed without the use of the new dissolved state calculations in MOOG. The continuous opacity at longer optical wavelengths, $\lambda>\lambda 4400$, is dominated by $\mathrm{H}^{-}$bound-free absorption, so we adopt the iron abundance derived from Fe I lines at these wavelengths as the standard to which all other values are corrected. These corrections and their uncertainties are listed in Table 10. We apply these corrections to abundances derived from all lines of other species, and the corrections are reflected in the values presented in Table 3 . The corrected iron abundances derived from Fe II lines show more scatter than the corrected iron abundances derived from $\mathrm{Fe}$ I lines, and this scatter dominates over any residual wavelength trends.

Our model atmosphere parameters do not change after making these corrections. We derived $T_{\text {eff }}$ by removing the correlation between iron abundance derived from Fe I lines and EP. Only the Fe I lines with measured EWs were used in this procedure.
Table 10

Mean Fe I Abundances Binned by Wavelength

\begin{tabular}{|c|c|c|c|c|}
\hline $\begin{array}{l}\text { Wavelength } \\
\text { Range ( } \AA \text { ) }\end{array}$ & $\langle\log \epsilon\rangle$ & Std. Dev & $N$ & Correction \\
\hline \multicolumn{5}{|c|}{ HD 108317} \\
\hline $2280-3100$ & 4.93 & 0.163 & 32 & +0.04 \\
\hline 3100-3647 & 4.81 & 0.093 & 107 & +0.16 \\
\hline $3647-4000$ & 4.87 & 0.081 & 40 & +0.10 \\
\hline $4000-4400$ & 4.92 & 0.074 & 27 & +0.05 \\
\hline $4400-6750$ & 4.97 & 0.070 & 94 & $\equiv 0.0$ \\
\hline \multicolumn{5}{|c|}{ HD 122563} \\
\hline 2280-3100 & 4.39 & 0.252 & 12 & +0.05 \\
\hline 3100-3647 & 4.17 & 0.112 & 69 & +0.27 \\
\hline $3647-4000$ & 4.26 & 0.090 & 27 & +0.18 \\
\hline $4000-4400$ & 4.39 & 0.055 & 17 & +0.05 \\
\hline $4400-6750$ & 4.44 & 0.075 & 86 & $\equiv 0.0$ \\
\hline \multicolumn{5}{|c|}{ HD 126238} \\
\hline $2280-3100$ & 5.39 & 0.197 & 10 & +0.13 \\
\hline $3100-3647$ & 5.28 & 0.117 & 76 & +0.24 \\
\hline $3647-4000$ & 5.37 & 0.108 & 22 & +0.15 \\
\hline $4000-4400$ & 5.46 & 0.066 & 13 & +0.06 \\
\hline $4400-6750$ & 5.52 & 0.082 & 96 & $\equiv 0.0$ \\
\hline \multicolumn{5}{|c|}{ HD 128279} \\
\hline $2280-3100$ & 4.91 & 0.172 & 28 & +0.11 \\
\hline $3100-3647$ & 4.83 & 0.096 & 96 & +0.19 \\
\hline $3647-4000$ & 4.91 & 0.070 & 32 & +0.11 \\
\hline 4000-4400 & 4.97 & 0.067 & 19 & +0.05 \\
\hline $4400-6750$ & 5.02 & 0.068 & 91 & $\equiv 0.0$ \\
\hline
\end{tabular}

This set of lines lies mostly in the redder region of the spectrum unaffected by the dip. (Most studies that adopt a spectroscopic $T_{\text {eff }}$ scale use redder sets of lines, also.) Figure 12 illustrates the relationship between iron abundance derived from all $\mathrm{Fe}$ I lines 


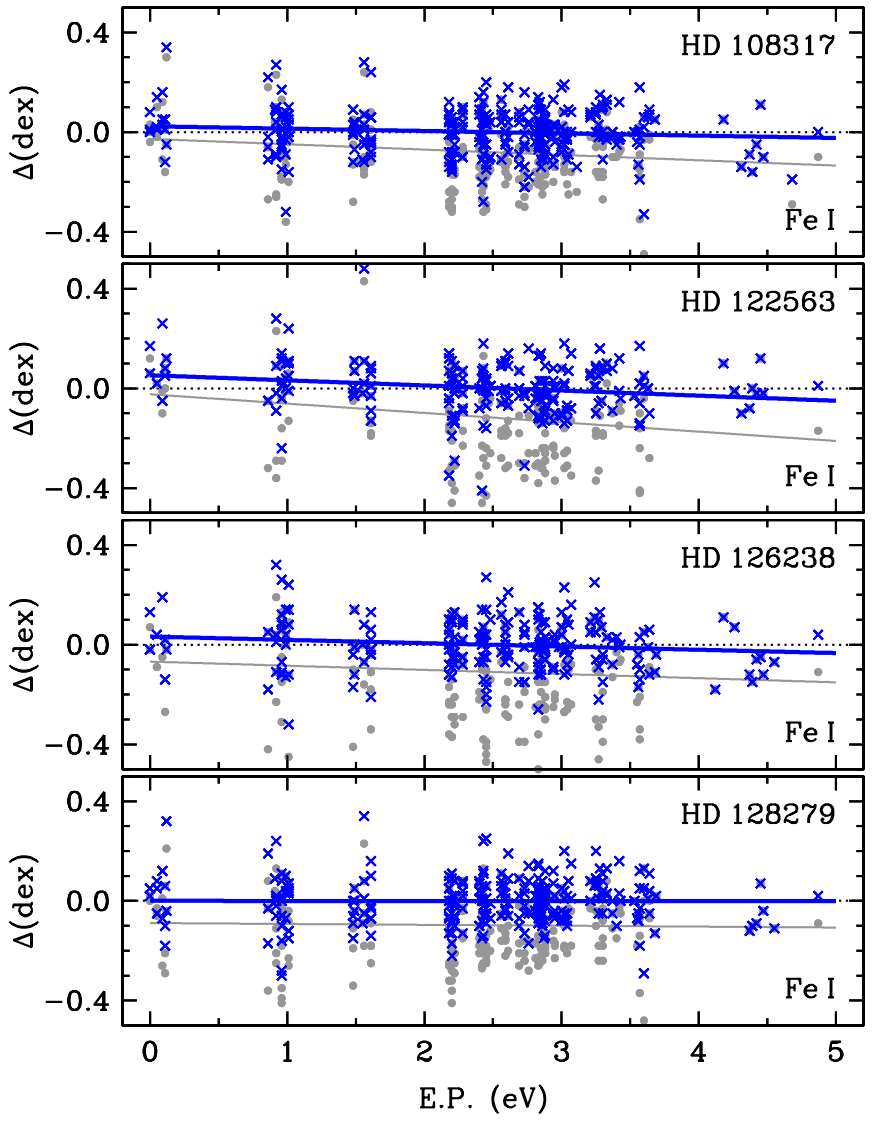

Figure 12. Line-by-line Fe I abundance deviations from the corrected mean as a function of EP. The gray circles denote the uncorrected abundances, and the blue crosses denote the corrected ones. The gray line marks the linear least-squares fit to the uncorrected abundances, and the bold blue line marks the fit to the corrected ones. The dotted black line marks an offset of zero from the mean iron abundance derived from Fe I lines.

(A color version of this figure is available in the online journal.)

and EP. The gray points illustrate the uncorrected abundances, and the gray lines mark the linear least-squares fits to them. The blue crosses illustrate the corrected abundances, and the bold blue lines mark the fits to them. Had we initially used all $\mathrm{Fe}_{\mathrm{I}}$ lines to derive $T_{\text {eff }}$, and not just the redder ones with measured EWs, these data would have suggested even cooler spectroscopic temperatures. The corrected Fe I abundances yield slopes consistent with zero (formally, the slopes are less than 2.6 times their errors), which supports our original spectroscopic $T_{\text {eff }}$ scale. In summary, this correction cannot be the explanation for the discrepancy between the spectroscopic and photometric values of $T_{\text {eff }}$ for these stars.

These corrections account for the small differences found between Tables 7 and 8 and the preliminary abundances presented in recent papers by our group. We proceed with caution and defer further investigation of this unexpected yet interesting effect to future work.

\section{RESULTS}

We have derived abundances or upper limits for 40 species of 37 elements heavier than zinc in these four stars. The final abundances and upper limits are reported in Tables 7 and 8 . The high $\mathrm{S} / \mathrm{N}$ at short wavelengths has allowed us to derive abundances from larger numbers of lines than have been useful previously and detect species whose only useful transitions
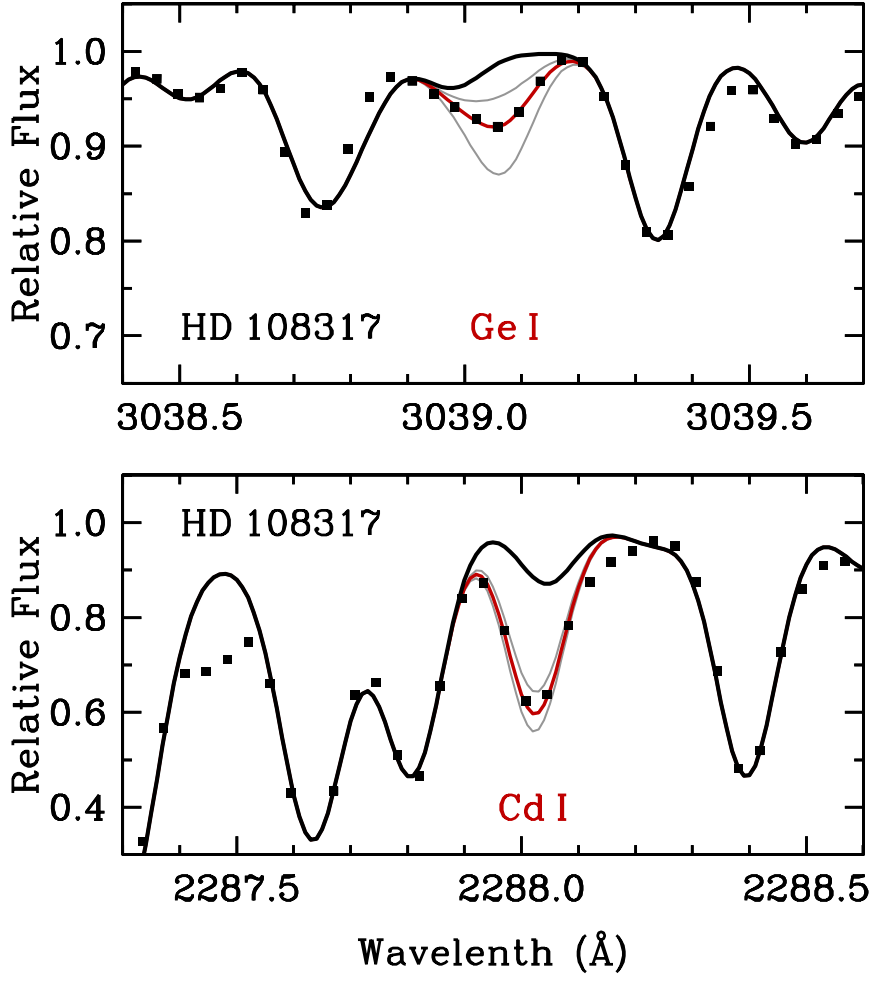

Figure 13. Comparison of observed and synthetic spectra in HD 108317. The Ge I $\lambda 3039$ and $\mathrm{Cd}_{\mathrm{I}} \lambda 2288$ lines are shown. The bold red line represents the best-fit abundance, the thin gray lines represent variations in this abundance by $\pm 0.30 \mathrm{dex}$, and the bold black line represents a synthesis with no germanium or cadmium.

(A color version of this figure is available in the online journal.)

are located shortward of $\approx \lambda 2650$ (e.g., Cd I, Te I, Lu II, Os II). Several of our syntheses of important NUV transitions are illustrated in Figures 13-15.

As discussed in detail in Appendix A, we reconsider the cadmium abundance previously derived from the lower $\mathrm{S} / \mathrm{N}$ spectrum of HD 122563 examined by Roederer et al. (2010b). $\mathrm{Cd} \mathrm{I}$ is detected at $\lambda 2288$, but it is blended, and our synthesis of this spectral region is a poor match to the observed spectrum. We report only an upper limit on the cadmium abundance in HD 122563.

We detect neutral and singly ionized species of osmium in HD 108317 and HD 126238. These abundances are not in agreement, with [Os I/Fe] higher by 0.54 and $0.53 \mathrm{dex}$, respectively. Roederer et al. (2010b) reported a smaller $(0.3$ dex) discrepancy in $\mathrm{BD}+17^{\circ}$ 3248. Adopting a different laboratory source for the Os II $\lambda 2282 \log (g f)$ value (Ivarsson et al. 2004 rather than Quinet et al. 2006) would only decrease the discrepancy by 0.09 dex. This offset is not due to missing energy levels in the Os I and II partition functions, and our tests indicate that it is not caused by neglecting isotope shifts in our synthesis. The source of this discrepancy remains unclear. Since only the neutral species of the neighboring elements iridium, platinum, and gold are detected, and since most previous osmium abundances have been derived using Os I, we adopt Os I as the primary osmium abundance indicator.

We are able to fit blends and derive more reliable abundances of the third $r$-process peak elements in HD 108317 by assuming that the third-peak elements in HD 128279 produce no absorption. The gold abundance derived from the Au I $\lambda 2675$ line should be viewed cautiously 

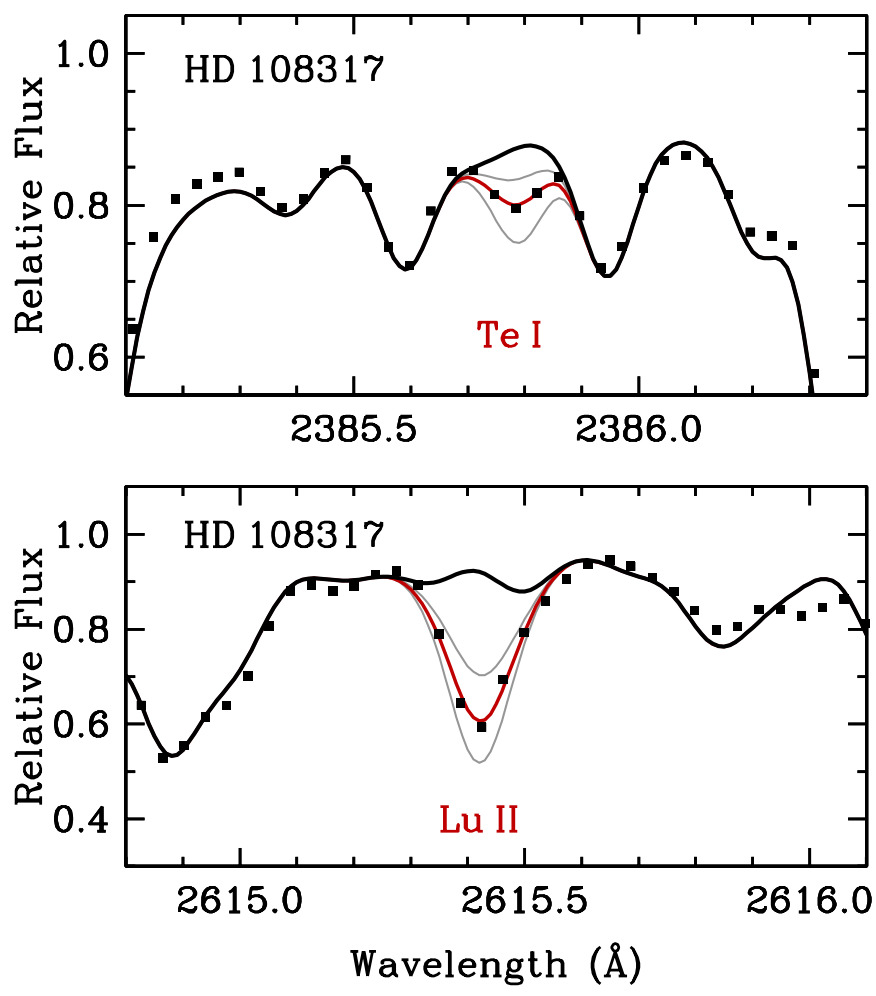

Figure 14. Comparison of observed and synthetic spectra in HD 108317. The Te I $\lambda 2385$ and Lu II $\lambda 2615$ lines are shown. The bold red line represents the best-fit abundance, the thin gray lines represent variations in this abundance by $\pm 0.30 \mathrm{dex}$, and the bold black line represents a synthesis with no tellurium or lutetium. As noted in Appendix A, strong Fe II lines at $\lambda \lambda 2383.04$ and 2388.63 depress the continuum around the Te I line.

(A color version of this figure is available in the online journal.)

given the strength of the blending features (Figure 15). We cannot exclude the possibility that a small amount of absorption from $\mathrm{PtI}$ is present in HD 128279. Relaxing this assumption would change the platinum abundances in HD 108317 by $<+0.1$ dex. Conservatively, we only report an upper limit on the platinum abundance in HD 128279.

We urge caution when interpreting abundances derived from weak, blended, or small numbers of lines. We have attempted to account for these factors in the stated uncertainties, but unidentified blends may lead to spuriously high abundances. The rhodium $(Z=45)$, silver $(Z=47)$, praseodymium $(Z=$ $59)$, terbium $(Z=65)$, lutetium, hafnium, osmium, iridium, and gold abundances are most susceptible to this bias.

Our abundances are not always in agreement with previous results for HD 122563. To some extent this reflects our higher quality spectrum and the greater number of lines available to us now than in the past; however, this explanation alone is insufficient. For example, Cowan et al. (2005) derived $\log \epsilon$ $(\mathrm{Ge})=-0.16$ from the Ge I $\lambda 3039$ line in HD 122563, while we derive $\log \epsilon(\mathrm{Ge})=-0.87$. We confirm that this discrepancy arises from the updated version of MOOG used for the analysis. (Rayleigh scattering has a $\lambda^{-4}$ dependence, so differences between the two versions of MOOG are most pronounced for transitions at short wavelengths.) Ratios constructed among the neutron-capture elements are in much better agreement. For example, after accounting for the different $\log (g f)$ values (all other atomic data-partition functions, etc.-are identical), Cowan et al. derived $[\mathrm{Zr} / \mathrm{Ge}]=+1.12$, while we derive
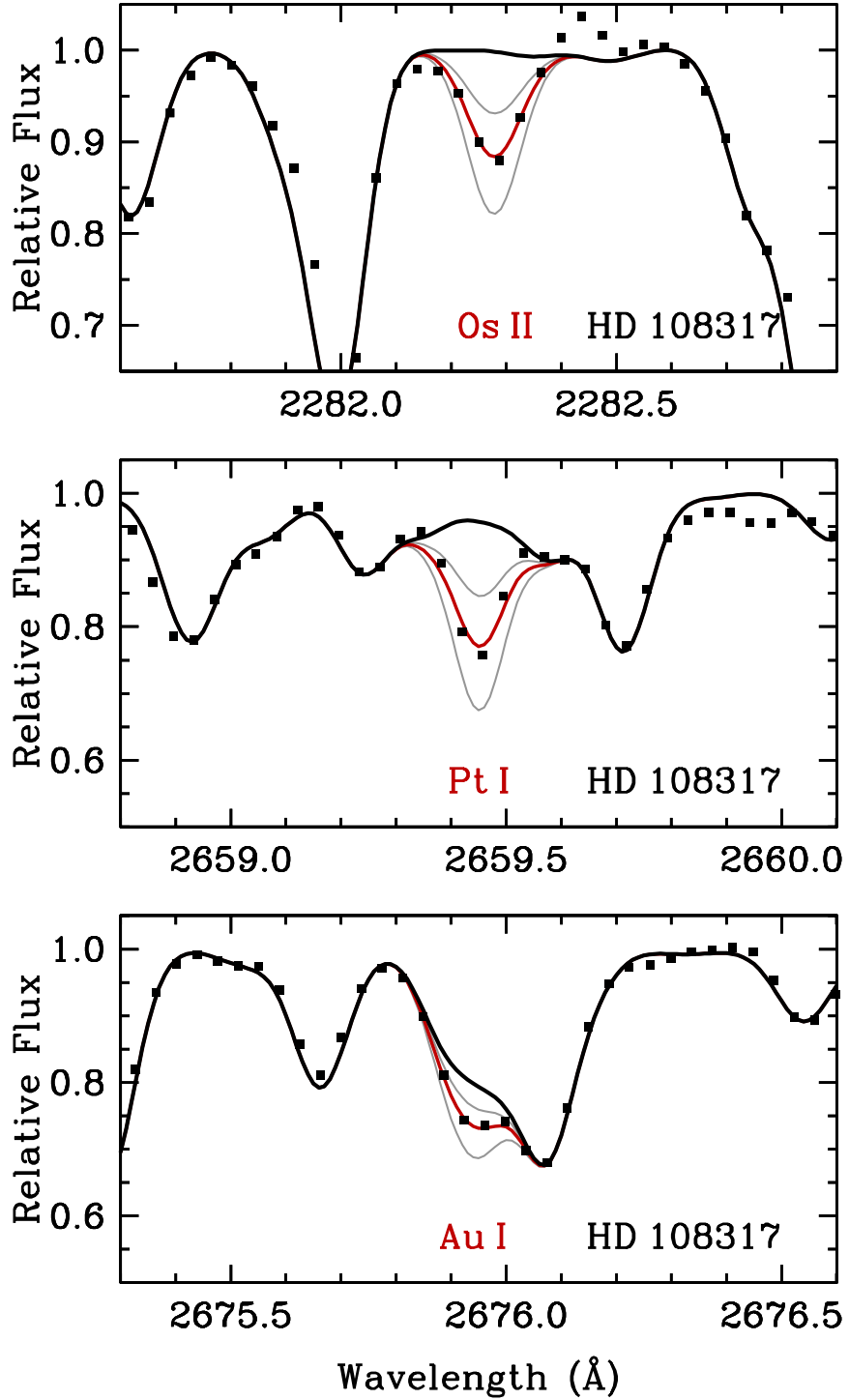

Figure 15. Comparison of observed and synthetic spectra in HD 108317. The Os II $\lambda 2282$, PtI $\lambda 2659$, and Au I $\lambda 2675$ lines are shown. The bold red line represents the best-fit abundance, the thin gray lines represent variations in this abundance by \pm 0.30 dex, and the bold black line represents a synthesis with no osmium, platinum, or gold.

(A color version of this figure is available in the online journal.)

$[\mathrm{Zr} / \mathrm{Ge}]=+1.14$. This agreement is encouraging. Nevertheless, the difference in $\log \epsilon$ illuminates the difficulty in obtaining reliable absolute abundances and underscores our assertion that abundance ratios are to be strongly preferred when interpreting the results.

\section{DISCUSSION}

Figures 16-19 illustrate the abundance pattern for each star. In these figures we compare the $\log \epsilon$ abundances with the predicted distributions of $s$ - and $r$-process material in the SS, scaled downward to match the stellar abundance levels. The predicted $s$ - and $r$-process distributions shown in Figures 16-19 are taken from Sneden et al. (2008). The lead and bismuth predictions are taken from the low-metallicity stellar models of Bisterzo et al. (2011) that reproduce the "strong" component of SS lead and bismuth. Recall that the SS $r$-process distribution implicitly includes contributions from all processes other than 


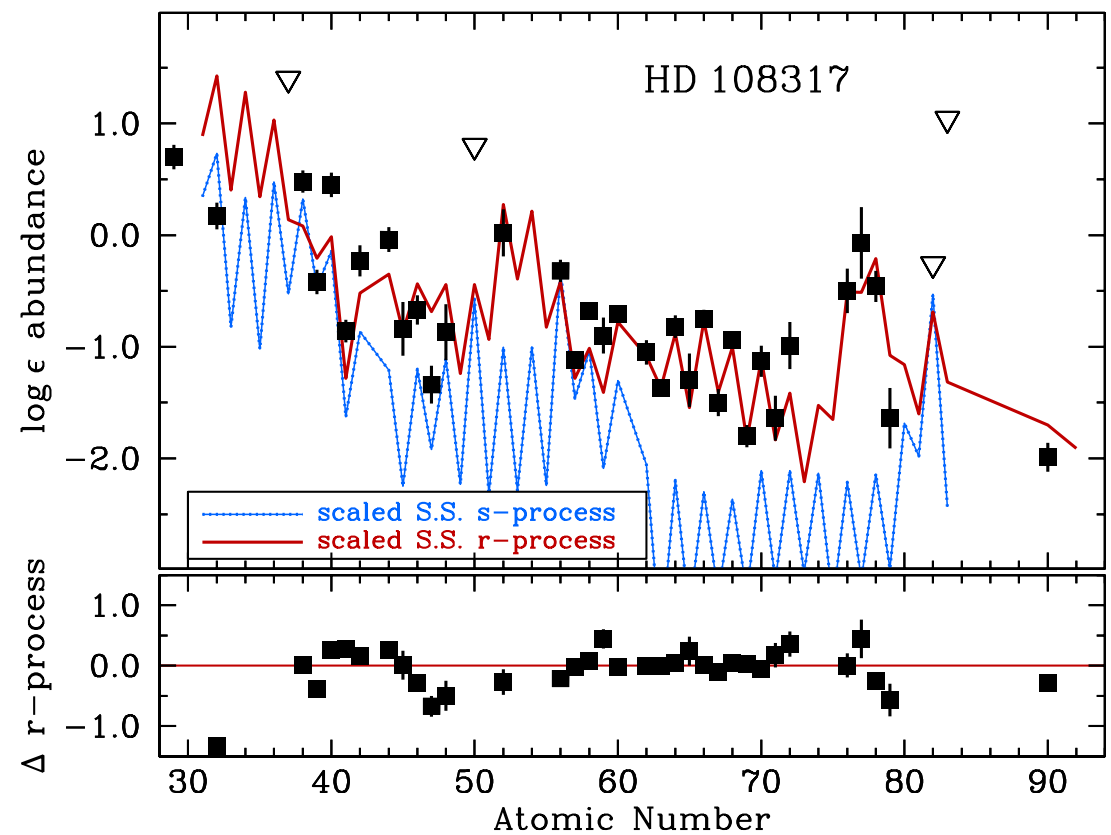

Figure 16. Comparison of the derived abundances in HD 108317 with the scaled SS $s$-process (studded blue line, normalized to Ba) and $r$-process (smooth red line, normalized to $\mathrm{Eu}$ ) abundance predictions. The top panel indicates the logarithmic abundances, and the bottom panel indicates the residuals between the stellar abundances and the $r$-process distribution when normalized to Eu. Filled squares indicate detections, and open downward-pointing triangles indicate upper limits. The Os II abundance has been omitted in favor of Os I. We advise that ratios of elements derived from different ionization states should be compared with caution.

(A color version of this figure is available in the online journal.)

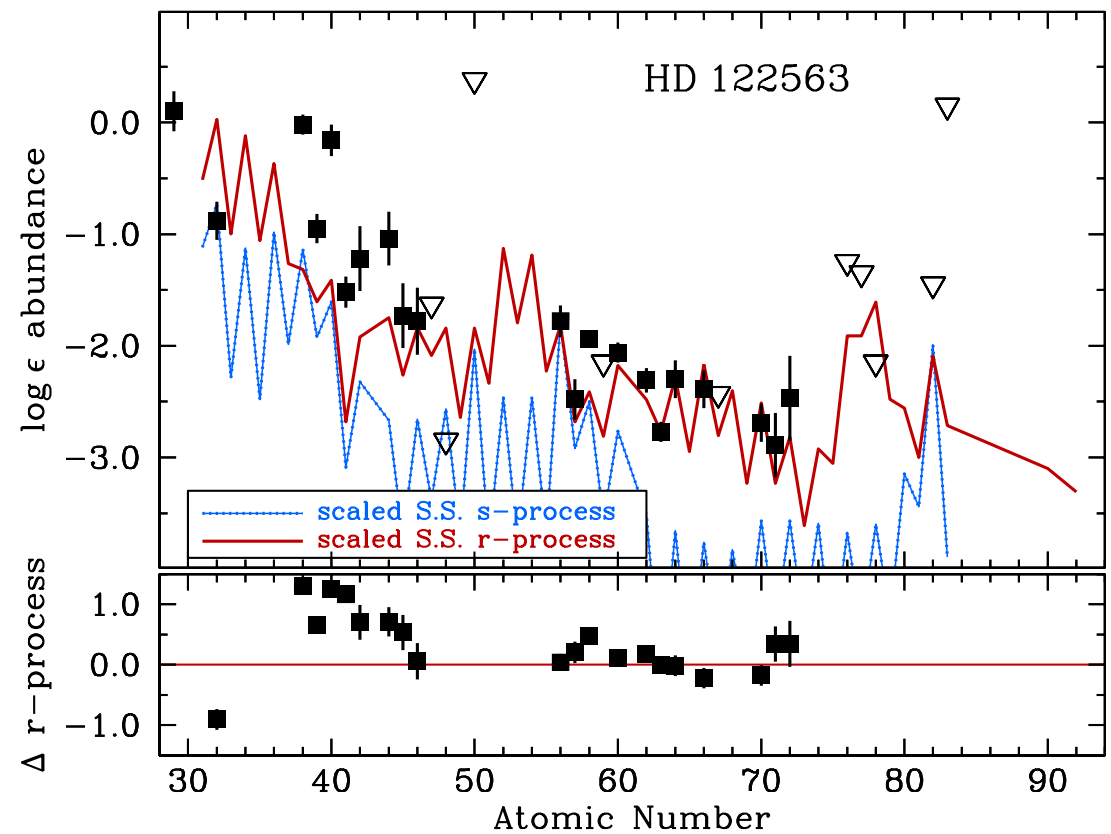

Figure 17. Comparison of the derived abundances in HD 122563 with the scaled SS $s$-process (studded blue line, normalized to Ba) and $r$-process (smooth red line, normalized to $\mathrm{Eu}$ ) abundance predictions. The top panel indicates the logarithmic abundances, and the bottom panel indicates the residuals between the stellar abundances and the $r$-process distribution when normalized to Eu. Filled squares indicate detections, and open downward-pointing triangles indicate upper limits. Honda et al. (2006) detected silver in HD 122563 in their higher quality spectrum. When compared with the adjacent element palladium, our upper limit is consistent with their detection. We advise that ratios of elements derived from different ionization states should be compared with caution.

(A color version of this figure is available in the online journal.)

the $s$-process, including an unspecified light-element primary process (LEPP; Travaglio et al. 2004).

Figures 16-19 reveal that the abundance patterns for $Z \geqslant 52$ in HD 108317, HD 126238, and HD 128279 (except for $Z>70$ in HD 128279) match the scaled SS $r$-process distribution more closely than the $s$-process distribution. The lighter elements also favor the $r$-process distribution but with greater element- to-element scatter than seen in the heavier elements. Fitting the observed abundance distributions with a combination of the SS $r$-process abundance pattern and a small additional amount of $s$-process material offers marginal improvement for HD 108317 and HD 126238. The lead abundances we have derived from an LTE analysis could be underestimated by factors of $\approx 2-3$ (Mashonkina et al. 2012). Consequently, according to Roederer 


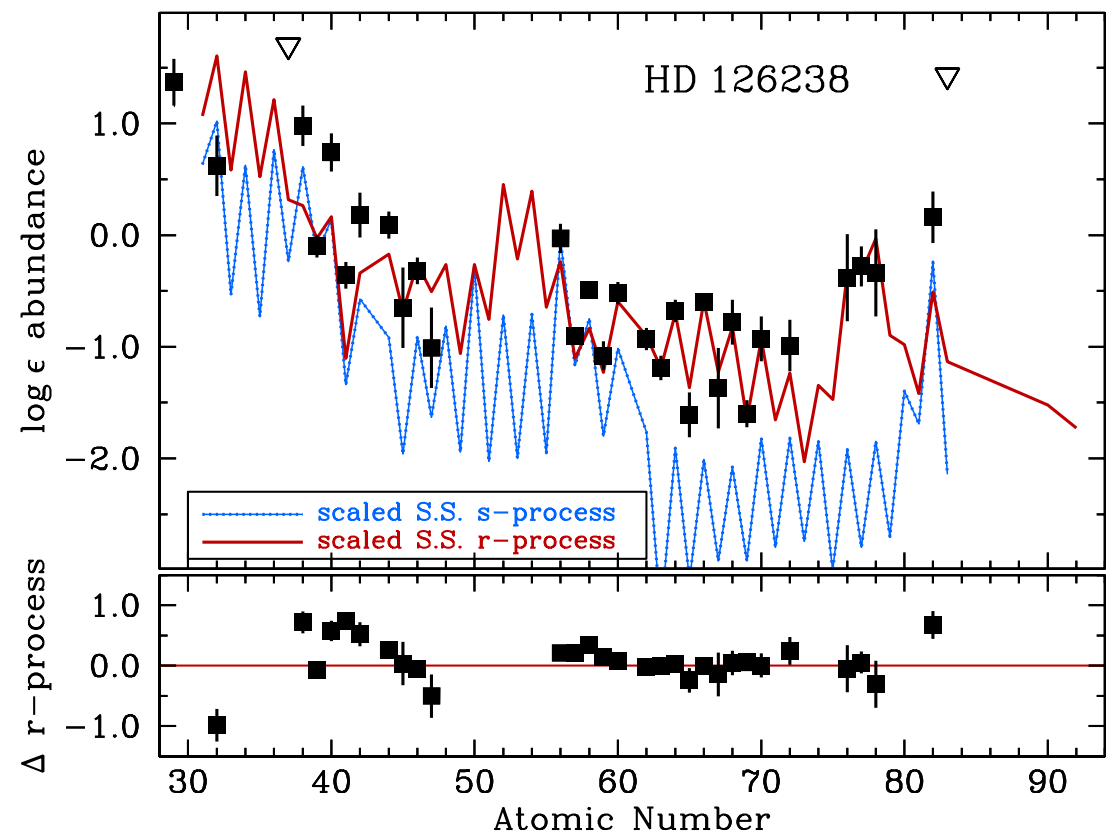

Figure 18. Comparison of the derived abundances in HD 126238 with the scaled SS $s$-process (studded blue line, normalized to Ba) and $r$-process (smooth red line, normalized to $\mathrm{Eu}$ ) abundance predictions. The top panel indicates the logarithmic abundances, and the bottom panel indicates the residuals between the stellar abundances and the $r$-process distribution when normalized to Eu. Filled squares indicate detections, and open downward-pointing triangles indicate upper limits. The Os II abundance has been omitted in favor of Os I. We advise that ratios of elements derived from different ionization states should be compared with caution.

(A color version of this figure is available in the online journal.)

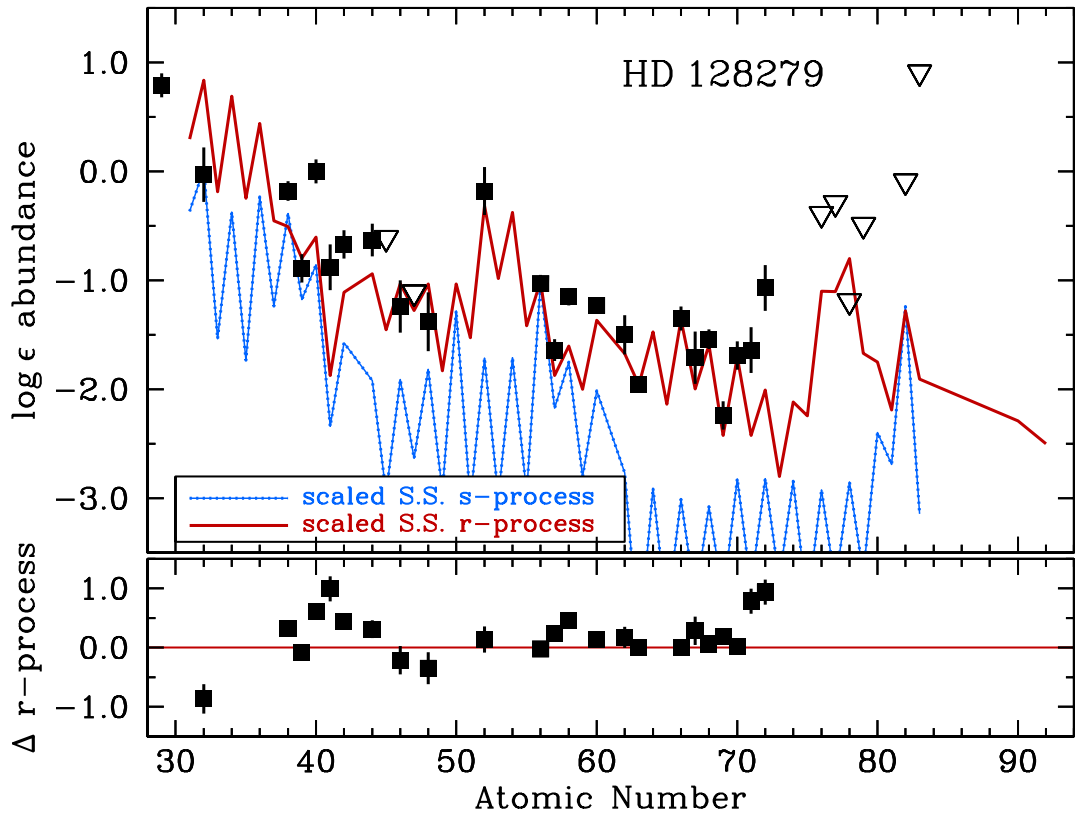

Figure 19. Comparison of the derived abundances in HD 128279 with the scaled SS s-process (studded blue line, normalized to Ba) and $r$-process (smooth red line, normalized to $\mathrm{Eu}$ ) abundance predictions. The top panel indicates the logarithmic abundances, and the bottom panel indicates the residuals between the stellar abundances and the $r$-process distribution when normalized to Eu. Filled squares indicate detections, and open downward-pointing triangles indicate upper limits. We advise that ratios of elements derived from different ionization states should be compared with caution.

(A color version of this figure is available in the online journal.)

et al. (2010a), the enhanced lead abundance in HD 126238 could indicate a small $s$-process contribution. The abundance pattern in HD 122563 clearly disfavors the $s$-process distribution, but it is not as well matched to the $r$-process distribution as the other stars are. No combination of $s$ - and $r$-process material can account for the observed abundance distribution in HD 122563, as has been noted previously (Sneden \& Parthasarathy 1983; Honda et al. 2006). HD 128279 is a probable member of a stellar stream. The more metal-rich stars in this stream do not show evidence of $s$-process enrichment (Roederer et al. 2010c), so it would be surprising if HD 128279 contained much $s$-process material.

We refrain from making any quantitative assessments of the $s$-process contamination. Fitting linear combinations of two distributions assumes that the SS distributions are representative at low metallicity and that no other processes contribute. There is ample evidence (e.g., Wasserburg et al. 1996; McWilliam 1998) that isotopes with $A \lesssim 130$ do not always adhere to the scaled 
SS $r$-process distribution when scaled to heavier isotopes. These elements, including strontium, yttrium, and zirconium, may be produced by nucleosynthesis channels separate from-but perhaps sometimes associated with—r-process nucleosynthesis. This complicates the interpretation of the $r$-process residual distribution for $A \lesssim 130$ isotopes. There is no single distribution produced by all $s$-process environments, and the resulting abundance ratios are known to vary depending on the neutron density, availability of ${ }^{13} \mathrm{C}$, timescales, number of dredge-up episodes, initial metallicity, etc. (e.g., Gallino et al. 1998; Bisterzo et al. 2010). Variations observed in the [La/Eu] ratio in stars with very low lead abundances $([\mathrm{Pb} / \mathrm{Eu}]<-0.7)$ also may point to intrinsic variations in $r$-process distributions (Roederer et al. 2010a). We cannot exclude the possibility of small amounts of $s$-process material in HD 108317 and HD 126238, but clear evidence of $s$-process contamination is not apparent in HD 122563 or HD 128279. Either way, the $s$ process contamination to many of the elements of interest in the present study is insignificant.

When detected, the ratios among palladium, silver, and cadmium are relatively constant. This agrees with the earlier results of Johnson \& Bolte (2002), Roederer \& Lawler (2012), and a substantially larger sample of stars examined by Hansen \& Primas (2011) and Hansen et al. (2012). The work of Hansen et al. suggests that palladium and silver are produced under $r$-process conditions that are different-less extreme neutron densities, perhaps-from the conditions that produce heavier mass nuclei, such as europium $(Z=63)$, via the main component of the $r$-process. Our results, as well as those of Roederer et al. (2010b) and Roederer \& Lawler (2012), imply that cadmium was likely produced along with palladium and silver in the events that enriched $\mathrm{BD}+17^{\circ} 3248$, HD 108317, HD 128279 , and HD 160617.

We recover the well-known downward trends of abundance with increasing atomic number between the first and second $r$-process peaks in HD 122563 (e.g., Truran et al. 2002; Honda et al. 2006; Montes et al. 2007). The contrast between HD 122563 and the stars discussed in the previous paragraph is illustrated in stark terms by the upper limit on the cadmium abundance in HD 122563 (Figure 17), which is more than 1 dex lower than the abundance of palladium. This indicates that production declined sharply through this mass range, yet some form of $r$-process nucleosynthesis seems to have produced the rare earth elements with masses between $A \approx 135$ and 180 .

The elements at the second and third $r$-process peaks in HD 108317 agree with the scaled SS $r$-process distribution within factors of $\approx 2$. Similar results have been found for the metal-poor halo stars BD $+17^{\circ} 3248$ and HD 160617 by Roederer et al. (2012) and Roederer \& Lawler (2012). While the number of halo stars with elements detected at both the second and third $r$-process peaks is still small, these results hint that tellurium was mainly produced along with the heavier $r$-process elements in the events that enriched these stars.

Upper limits on the platinum in HD 122563 and HD 128279 suggest that the third $r$-process peak elements here are deficient relative to the scaled SS $r$-process distribution. Are these patterns indicative of separate nucleosynthesis processes, or are they the outcomes of high- and low-intensity $r$-process nucleosynthesis? Kratz et al. (2007) have used a set of siteindependent waiting-point $r$-process calculations to show that the combined yields of exposures with different neutron densities can reproduce the overall shape of the SS $r$-process distribution from the first $r$-process peak to the actinides. Those calcula- tions suggest that neutron densities lower than $n_{n} \sim 10^{24}-10^{26}$ could under-produce the third peak elements. On the other hand, Travaglio et al. (2004), Montes et al. (2007), and Qian \& Wasserburg (2007) have suggested that mixtures of two generic nucleosynthesis events (a main $r$-process and an LEPP) can explain the stellar abundance patterns (see also Qian \& Wasserburg 2008), including the lack of a correlation between LEPP enhancement and overall europium enrichment. Our new abundance derivations, particularly our detections and upper limits of elements at the second and third $r$-process peaks, may aid theoretical explorations of these issues.

\section{SUMMARY}

We have detected up to 34 elements produced by neutroncapture reactions in each of HD 108317, HD 122563, HD 126238, and HD 128279. The unique capability of highresolution spectroscopy in the NUV with HST and STIS has enabled us to detect seven of these 34 elements that cannot be detected from the ground. HD 108317 and the strongly $r$-process-enriched star $\mathrm{BD}+17^{\circ} 3248$, itself the subject of numerous ground- and space-based abundance studies (including, e.g., Burris et al. 2000; Cowan et al. 2002, 2005; Den Hartog et al. 2005; François et al. 2007; Sneden et al. 2009; Roederer et al. 2010b, 2012), are the two metal-poor halo stars with the largest number of heavy elements detected.

We explore several potential sources of systematic uncertainties in the abundance analysis. Our $T_{\text {eff }}$ estimates, derived from standard spectroscopic techniques, are consistently more than $200 \mathrm{~K}$ cooler than $V-K$ photometric estimates, and for HD 122563 our spectroscopic $T_{\text {eff }}$ is $150 \mathrm{~K}$ cooler than the $T_{\text {eff }}$ derived from a recent measurement of the stellar radius. In general, abundance ratios are more reliable than absolute abundances. Ratios constructed among elements whose abundances have been derived from species in the same ionization state are more reliable than ratios mixing neutral atoms and ions. Our iron abundances reveal wavelength-dependent abundance trends that cannot be explained by non-LTE effects and may indicate shortcomings in the calculation of the continuous opacity. We are continuing to investigate this matter. We make empirical corrections for this effect, but we encourage those who wish to make use of our derived abundances to exercise due respect for these systematic uncertainties.

We find that some form of $r$-process nucleosynthesis is responsible for the heavy elements in these stars, though we cannot exclude the possibility of a small trace of $s$-process material in HD 108317 and HD 126238. Our results, combined with previous results for $\mathrm{BD}+17^{\circ} 3248$ and HD 160617, reveal the $r$-process nucleosynthesis patterns in six stars with $r$-process enrichment levels spanning a range of a factor of 40 : $[\mathrm{Eu} / \mathrm{Fe}]=$ $+0.9,+0.5,+0.4,+0.2,0.0$, and -0.7 in $\mathrm{BD}+17^{\circ} 3248$, HD 108317, HD 160617, HD 126238, HD 128279, and HD 122563, respectively. The enrichment levels of the lighter elements, like strontium, yttrium, and zirconium, vary only by factors of 2-8. The relative heights of the second and third $r$-process peaks are broadly consistent with the scaled SS $r$-process distribution in $\mathrm{BD}+17^{\circ} 3248$, HD 108317, and HD 160617. There is a general decrease in abundance with atomic number in HD 122563, especially between the first and second $r$-process peaks, that differs from the other stars examined.

While our new observations are no doubt useful to identify the source(s) of the heavy elements in these four stars, their greater impact surely lies in their ability to inform models of 
$r$-process nucleosynthesis. Our work has demonstrated that several previously undetected elements key to this understanding can be detected and reliably measured in ancient halo stars. We trust that these new observational constraints will be of use in theoretical explorations of $r$-process nucleosynthesis.

We express sincere appreciation to G. Preston for obtaining some of the MIKE observations, C. J. Hansen for sending results in advance of publication, K.-L. Kratz and F. Montes for helpful discussions, and the anonymous referee for several suggestions that we believe have improved this paper significantly. We also appreciate the expert assistance of the STScI staff in obtaining these observations and the astronauts of STS-125 for their enthusiastic return to HST for Servicing Mission 4.

This research has made use of NASA's Astrophysics Data System Bibliographic Services, the arXiv pre-print server operated by Cornell University, the SIMBAD and VizieR databases hosted by the Strasbourg Astronomical Data Center, the Atomic Spectra Database hosted by the National Institute of Standards and Technology, the Mikulski Archive at the Space Telescope Science Institute, and the NUV and visible spectral atlases of Arcturus and the Sun (Hinkle et al. 2000, 2005). IRAF is distributed by the National Optical Astronomy Observatories, which are operated by the Association of Universities for Research in Astronomy, Inc., under cooperative agreement with the National Science Foundation. This publication makes use of data products from the Two Micron All Sky Survey, which is a joint project of the University of Massachusetts and the Infrared Processing and Analysis Center/California Institute of Technology, funded by the National Aeronautics and Space Administration and the National Science Foundation. We recognize and acknowledge the very significant cultural role and reverence that the summit of Mauna Kea has always had within the indigenous Hawaiian community. We are most fortunate to have the opportunity to conduct observations from this mountain.

Generous support for Program number 12268 was provided by NASA through a grant from the Space Telescope Science Institute, which is operated by the Association of Universities for Research in Astronomy, Inc., under NASA contract NAS 5-26555. I.U.R. is supported by the Carnegie Institution for Science through a Carnegie Fellowship. J.E.L. acknowledges support from NASA grant NNX10AN93G. T.C.B. and H.S. acknowledge partial support from grants PHY 02-16783 and PHY 08-22648: Physics Frontier Center/Joint Institute for Nuclear Astrophysics (JINA), awarded by the U.S. National Science Foundation (NSF). H.S. acknowledges additional support from NSF grant PHY-1102511. C.S. acknowledges support from NSF grant AST 09-08978.

Facilities: HST (STIS), Keck I (HIRES), Magellan:Clay (MIKE)

\section{APPENDIX A}

\section{COMMENTS ON NUV LINE SELECTION AND OSCILLATOR STRENGTHS}

Blending from atomic and molecular contaminants must be carefully assessed to reliably identify absorption due to heavy elements in the NUV spectra of metal-poor stars. Very few transitions in the NUV are unblended, so our abundance analysis must proceed by spectral synthesis. Our initial synthesis line lists include all atomic and molecular $(\mathrm{OH})$ transitions in the Kurucz $\&$ Bell (1995) line lists. We update the $\log (g f)$ values with experimental data when known. Here, we discuss the heavy- element lines of interest in our STIS spectra and lines that blend with them, as revealed by the observed line profiles. The $\log (g f)$ values of blending features come from the Kurucz \& Bell lists unless noted otherwise. Confidence in the accuracy of these values should be tempered.

Zinc $(Z=30)$. Zinc is traditionally considered an iron group element, but neutron-capture reactions must pass through zinc isotopes when running from lighter, more abundant iron group elements to the heavy elements. A few good Zn I transitions exist in the optical spectral range. We report an additional Zn I transition at $\lambda 3075.90$ found in our STIS spectra. This transition is blended with an Fe I line at $\lambda 3075.72(\log g f=-0.68)$ and a weak V I transition at $\lambda 3075.93(\log g f=-0.62)$. The abundance derived from this $\mathrm{Zn}$ I transition agrees well with the abundance derived from the $\lambda \lambda 4680,4722$, and 4810 transitions.

Germanium $(Z=32)$. The Ge I transition at $\lambda 2651.17$ is situated between several manageable blending features, most notably an $\mathrm{OH}$ line at $\lambda 2651.30(\log g f=-2.88)$. These blends are too strong to derive a reliable Ge I abundance in HD 126238. A nearby, but weaker, Ge I transition at $\lambda 2651.57$ is too blended for abundance work in all four stars. The Ge I transition at $\lambda 2691.36$ is relatively clean, although unidentified absorption lines in each wing (2691.20 and $\lambda 2691.48$, the former itself in the wing of a strong CrII line at $\lambda 2691.04 ; \log g f=-0.40$ ) are present in all four stars. This line gives abundance results lower by $0.1-0.5$ dex than all other Ge I lines considered, but we have no other compelling reason to exclude it as an abundance indicator. The Ge I transition at $\lambda 3039.07$ has been used previously for abundance work, and we can model the only minor blending feature, a weak Fe I line at $\lambda 3038.98$ with no $\log (g f)$ value given in the NIST database. (The Kurucz \& Bell 1995 lists give $\log g f=-2.59$, which we adjust from -2.0 to -2.4 to fit the observed line profile.) Moderately strong Ge I absorption lines are also detected at $\lambda \lambda 2591.17$ and 2754.59. Blends with $\mathrm{OH}$ and $\mathrm{Fe}$, respectively, in addition to severe line blanketing from a nearby strong Fe II line at $\lambda 2755.74$ in HD 122563 and HD 126238, render them unusable for abundance work.

Zirconium $(Z=40)$. We have identified eight $\mathrm{Zr}$ II transitions in our STIS spectra (in addition to another 30 or so in the optical regime) that are reliable abundance indicators in these stars. The $\lambda 2567.64$ transition is unblended except in HD 122563, where line blanketing from the nearby strong Fe II $\lambda 2566.91$ transition depresses the continuum. The $\lambda 2699.60$ transition suffers mild blends in the wings that can be fit in all stars in our sample except for HD 122563, where extra absorption is present on the red side of the line. Our syntheses suggest that the $\lambda 2700.13$ transition contains a mild $(<10 \%) \mathrm{OH}$ blend on the blue side of the line and even weaker blends on the red side from $\mathrm{Fe}_{\mathrm{I}}$ and II and Co I. The $\mathrm{S} / \mathrm{N}$ and resolution of our spectra are sufficient to fit these blends. The $\lambda 2732.72$ transition is clean in HD 108317 and HD 128279, but the placement of the continuum is too uncertain in HD 122563 and HD 126238 to yield a reliable abundance. The $\lambda 2758.81$ transition is largely unblended in HD 108317, HD 122563, and HD 128279. Our syntheses suggest that it is significantly blended with the V II $\lambda 2758.82$ transition $(\log g f=-0.54$ in the Kurucz \& Bell 1995 lists) in HD 126238, where it introduces a slight asymmetry in the line profile. We can find no laboratory $\log (g f)$ value for this transition. This V II absorption is expected to be no more than a minor contaminant to the $\mathrm{Zr}$ II line in the other stars. The $\lambda 2915.99$ transition lies on the blue wing of a moderately strong Fe II transition at $\lambda 2916.15$, which we can fit; NIST quotes $\log (g f)=-3.31$ 
for this line with an uncertainty of $50 \%$. Our syntheses suggest that the $\mathrm{Fe}$ II line is also blended with $\mathrm{OH}$ at $\lambda 2916.23(\log g f$ $=-2.26)$. We derive an abundance from this $\mathrm{Zr}$ II transition in all four stars. The $\lambda 3054.84$ transition is blended with Co II $\lambda 3054.72(\log g f=-2.74)$ and $\mathrm{OH} \lambda \lambda 3054.97+3054.99$ in each wing $(\log g f=-2.72$ and -3.12 , respectively), but these blends can be reasonably fit in all four stars. The $\lambda 3095.07$ transition is unblended in all four stars.

The $\lambda 2639.08$ transition is unblended, but its observed wavelength is approximately $\lambda 0.01-0.03$ redder (i.e., to longer wavelengths) than predicted in all four stars. In HD 126238, the most metal-rich star in our sample, this line yields an abundance higher by a factor of four than the mean of all other $\mathrm{Zr}$ II lines. There are no obvious iron group blending transitions in the NIST database or the Kurucz \& Bell (1995) lists, but due to the possibility of an unidentified blend, we discard this line from consideration in all stars in the sample. We also examined $\mathrm{Zr}$ II transitions at $\lambda \lambda 2571.39,2571.46,2752.20,2968.96$, $2969.62,3030.92,3036.39$, and 3036.50. All were found to be too blended for use here. The $\lambda 3061.33$ transition is unblended in these stars, but it was not covered in either of the Ljung et al. (2006) or Malcheva et al. (2006) laboratory studies. The Kurucz $\&$ Bell database reports $\log (g f)=-1.38$. Using the zirconium abundance derived from the other lines in these stars, we determine an empirical $\log (g f)$ value for the $\lambda 3061.34$ line of -1.24 \pm 0.12 . We do not use this line in our analysis.

We have performed a detailed comparison of the $\mathrm{Zr}$ II $\log (g f)$ values presented by Ljung et al. (2006) and Malcheva et al. (2006) for all of the transitions used in our analysis. It is reassuring that the mean zirconium abundance derived for each star using different sets of $\log (g f)$ values varies by no more than 0.04 dex. The standard deviations of these measurements are $0.13-0.22$ dex. These values are slightly larger than the standard deviations of the differences in the $\log (g f)$ values between the two studies, 0.07-0.18 dex depending on which set of stellar lines is considered. Neither study covers all of the transitions we have examined, unfortunately. Malcheva et al. suggest that their transition probabilities are accurate to within $20 \%$, while Ljung et al. estimate uncertainties of $4 \%-11 \%$ for the transitions we have analyzed. Based on this, we adopt the Ljung et al. transition probabilities whenever possible. Five transitions in our list have highly discrepant $\log (g f)$ values (differences of $0.32-0.60$ dex) between the two studies, and for these we choose the $\log (g f)$ value that yields a zirconium abundance nearer the mean abundance derived from other $\mathrm{Zr}$ II transitions. All four stars in our sample consistently point to the same preferred $\log (g f)$ values. Four of the five transitions $(\lambda \lambda 2732.72,3344.79$, 3403.68 , and 3549.51) favor the Ljung et al. values, and one (2699.60 A) favors the Malcheva et al. value.

Niobium $(Z=41)$. The $\mathrm{Nb}$ II transition at $\lambda 2950.88$ shows absorption in both wings from $\mathrm{OH}$ at $\lambda \lambda 2950.76$ and 2950.94 ( $\log g f=-2.33$ and -3.23 , respectively). Treating the overall $\mathrm{OH}$ abundance as a free parameter, we can fit these blends, and we use this niobium abundance indicator in all four stars. The $\lambda 3028.44$ transition suffers only a blend in the blue wing with an unidentified absorption feature. The NbII absorption is too weak to detect in HD 128279, but otherwise it gives results in good accordance with the $\lambda 2950.88$ transition. The Nb II $\lambda 2827.08$ line is too blended in these stars. We detect what appears to be $\mathrm{Nb}$ II absorption at $\lambda \lambda 2876.96$ +2877.04 , but there are too many blending features to reliably identify the continuum or derive a meaningful niobium abundance.
Molybdenum $(Z=42)$. The line profile of the Mo II $\lambda 2871.51$ transition clearly indicates a blend, which may be due to $\mathrm{Fe}$ I $\lambda 2871.48$. Unfortunately, no experimental $\log (g f)$ value is known for this transition. The Kurucz \& Bell (1995) lists give $\log (g f)=-2.78$. It is too blended to fit empirically. We only derive an upper limit on the molybdenum abundance from this Mo II line.

Cadmium $(Z=48)$. Our syntheses suggest that only one line blends with the $\mathrm{Cd}$ I $\lambda 2288.02$ transition, an Fe I transition at $\lambda 2288.04$ present in the Kurucz line lists $(\log g f=-3.58)$. Our line list can provide a reasonable match to the observed spectrum in HD 108317 and HD 128279. We derive a cadmium abundance in these two stars and estimate uncertainties by empirically varying the blending $\mathrm{Fe}_{\mathrm{I}}$ line as far as allowed by the line profile. The lower $\mathrm{S} / \mathrm{N}$ and higher line density in HD 126238 at $2288 \AA$ prevent us from reliably identifying the continuum, unfortunately, so we cannot derive a meaningful cadmium abundance in this star. Our synthesis provides a poor match to the overall spectral region surrounding the $\mathrm{Cd}$ I line in HD 122563, and the relatively high line density in this region, due to the much cooler temperatures, also makes it difficult to identify the continuum. We reported a tentative detection of Cd I from this transition in Roederer et al. (2010b), but our higher $\mathrm{S} / \mathrm{N}$ data indicate that an upper limit may be more appropriate.

Tellurium $(Z=52)$. The Te I $\lambda 2385.79$ transition is weak but lies in a relatively clean spectral window. Strong Fe II lines at $\lambda 2383.06(\log g f=-1.29$ according to NIST) and $\lambda 2388.63$ ( $\log g f=-0.14$ according to NIST) depress the continuum by about $10 \%$ in HD 108317 and HD 128279, which is still manageable. In HD 122563 and HD 126238, the continuum is depressed by $40 \%-70 \%$, rendering the Te I line useless as an abundance indicator. In HD 108317 and HD 128279, nearby Fe I transitions at $\lambda 2385.59(\log g f=-2.95)$ and $\lambda 2385.92$ $(\log g f=-4.15)$ can be fit without significantly disturbing the line profile of the Te I transition. The only other minor blending feature suggested by our syntheses is a weak $\mathrm{Cr}$ I line at $\lambda 2385.72$. The NIST database gives $\log (g f)=-0.88$ for this line with a stated uncertainty of $50 \%$, and we fit the overall line profile while varying the strength of this $\mathrm{Cr}$ I line. Even accounting for the sub-solar $[\mathrm{Cr} / \mathrm{Fe}]$ ratios, the line profile still demands that the $\log (g f)$ of this line should be weaker, from -1.5 to -1.7 based on these observations.

Lutetium $(Z=71)$. The continuum surrounding the Lu II ground-state transition at $\lambda 2615.42$ is depressed by about $5 \%$ by neighboring Fe II lines at $\lambda 2613.82(\log g f=-0.36$ according to NIST) and $\lambda 2617.62(\log g f=-0.52$ according to NIST) in HD 108317 and HD 128279. The line profile also suggests minor blends from Co I at $\lambda 2615.33(\log g f=-0.99$, which we adjust to -1.5 assuming a solar $[\mathrm{Co} / \mathrm{Fe}]$ ratio) and $\mathrm{OH}$ at $\lambda 2615.50(\log g f=-3.90)$, which can be fit moderately well. In HD 122563 the continuum is depressed by about 20\%, and we report a tentative lutetium abundance in this star. The continuum is depressed by about 30\% in HD 126238 and the blending is more severe, so we are unable to derive an abundance here even though the Lu II line is strong and easily detected. We present the hfs pattern for the Lu $\lambda 2615$ transition in Appendix B.

Hafnium $(Z=72)$. The Hf II $\lambda 2641.41$ transition is detected in all four stars as a weak absorption asymmetry between weaker Fe I transitions at $\lambda 2641.30(\log g f=-2.47$, according to the Kurucz \& Bell 1995 lists) and $\lambda 2641.49(\log g f=-1.67$, according to the Kurucz \& Bell lists). Laboratory $\log (g f)$ values are not known for either of these blends, but we can adjust them to produce reasonable fits to the line profile (from -2.47 to -3.0 
for the former, and from -1.9 to -2.1 for the latter). The Hf II line is extremely weak in HD 122563. Including hafnium in our synthesis does improve the fit to the absorption line profile at this wavelength, so we report a tentative detection.

Osmium $(Z=76)$. The Os II transition at $\lambda 2282.28$ is unblended in both HD 108317 and HD 128279, although we do not detect any absorption from osmium in HD 128279. This transition is clearly detected and still relatively unblended in HD 126238, suffering only a few minor blends, the most notable arising from an unidentified absorption feature at $\lambda 2282.15$. The $\mathrm{S} / \mathrm{N}$ is relatively low at $\lambda 2282$, so we report a tentative osmium abundance in HD 126238. No absorption is apparent at $\lambda 2282.28$ in HD 122563. Our syntheses have difficulty reproducing the overall observed spectrum in this region in HD 122563 (see discussion regarding $\mathrm{Cd}$ I). The Os I transition at $\lambda 3058.66$ lies between two stronger lines of $\mathrm{Fe}$ I at $\lambda 3058.49(\log g f=-0.50$, which we adjust from -1.0 to -1.1$)$ and $\lambda 3059.09(\log g f$ $=-0.66$, which we adjust from -0.95 to -1.1$)$. There is no obvious absorption feature at this wavelength in HD 128279 , but we can improve the fit slightly by adjusting the strength of a very weak Fe I line at $\lambda 3058.70(\log g f=-3.51)$. In HD 108317, where absorption from Os I is detected, this Fe I blend serves to reduce the fraction of the absorption due to Os I. The Os I $\lambda 3058$ transition gives consistently higher abundances than the Os II $\lambda 2282$ transition. If we do not adjust the strength of the Fe I blend, however, our derived Os I abundance would be even higher in HD 108317. We include this adjusted Fe I line in the syntheses for the remaining stars in our sample. We derive an osmium abundance from the $\lambda 3058$ line in HD 126238 but not HD 122563, where no absorption is detected. We adopt the $\log (g f)$ value for the Os II $\lambda 2282$ transition from Quinet et al. (2006), so that the Os I and Os II $\log (g f)$ values are drawn from a common source. Adopting instead the $\log (g f)$ value from Ivarsson et al. (2004) would have increased the derived abundance by only 0.09 dex.

Iridium $(Z=77)$. Only the Ir I transition at $\lambda 2924.79$ is strong enough to detect in HD 108317 and HD 126238. No absorption is detected at this wavelength in HD 122563 and HD 128279. The Ir I line is significantly blended with the red wing of a strong composite feature dominated by V II $\lambda 2924.64$ $(\log g f=+0.15)$, which can be fit well. HD 108317 shows extra absorption at the correct wavelength relative to HD 128279 if the absorption arises from Ir I, which is encouraging, but our derived iridium abundances should be viewed with caution.

Platinum $(Z=78)$. The $\mathrm{Pt} \mathrm{I}$ transition at $\lambda 2659.45$ is blended with several weaker features, including Cr II $\lambda 2659.46$ $(\log g f=-0.94$, which we adjust to -0.3 assuming a solar $[\mathrm{Cr} / \mathrm{Fe}]$ ratio) and $\mathrm{OH} \lambda 2659.58(\log g f=-3.04)$. The strength of these lines can be fit assuming that none of the absorption at $\lambda 2659.45$ in HD 128279 comes from Pt I. If the $\log (g f)$ of the Cr II line-which is only found in the Kurucz lists - is assumed to be significantly lower, then the absorption could be due to Pt I in HD 128279. The HD 122563 spectrum is consistent with no absorption from either Cr II or Pt I here, so the weak line in HD 128279 might be due to Pt I. We conservatively adopt only an upper limit on platinum in HD 128279. This Pt I line is easily detected in HD 126238, but the blending features are far too strong in this star to derive a reliable platinum abundance.

The Pt I transition at $\lambda 2929.78$ is blended with a $\mathrm{Cr}$ II transition at $\lambda 2929.80$, for which no laboratory $\log (g f)$ is available. (The Kurucz \& Bell 1995 lists give $\log g f=-0.03$, which we adjust to -0.8 assuming a solar $[\mathrm{Cr} / \mathrm{Fe}]$ ratio.) We can fit the strength of this line in HD 128279, assuming that none of the absorption arises from $\mathrm{Pt} \mathrm{I}$, and use that to derive a platinum abundance in HD 108317 and HD 126238. No absorption is detected here in HD 122563. The Pt I $\lambda 2646.88$ transition is blended with an unidentified absorption feature at $\lambda 2646.85$ present in all of our spectra, including HD 122563 and HD 128279. After accounting for this absorption in our line list, only a very small amount of extra absorption is visible in HD 108317 and HD 126238 that presumably comes from Pt I. We discard this line from further consideration. There is an unidentified absorption feature at the exact wavelength of the Pt I $\lambda 2771.66$ transition. This absorption does not change strength between HD 108317 and HD 128279 as all other lines of heavy elements do, so we assume that it is not due to Pt I. This line and the Pt I $\lambda 2650.85$ lines originate from different lower levels of $0.10 \mathrm{eV}$; Den Hartog et al. (2005) determined the $\log (g f)$ value of the $\lambda 2650.85$ transition to be 0.13 dex higher than that of the $\lambda 2771$ transition. We cannot detect the $\lambda 2650$ transition, so it is reasonable to conclude that the absorption at $\lambda 2771.66$ is not due to $\mathrm{Pt}$ I. The Pt I transition at $\lambda 2997.96$ is blended with strong $\mathrm{OH}$ transitions at $\lambda 2997.96$ $(\log g f=-2.07)$ and $\lambda 2998.02(\log g f=-2.26)$, preventing us from deriving a platinum abundance from this transition in any star in the sample.

Gold $(Z=79)$. The Au I $\lambda 2675.94$ transition is detected in HD 108317 as extra absorption relative to HD 128279. There are a number of blends to this line, including $\mathrm{OH} \lambda 2675.89$ $(\log g f=-3.15$, according to the Kurucz \& Bell 1995 lists), Co I $\lambda 2675.98(\log g f=-1.66$, according to the Kurucz \& Bell lists), and Ti I $\lambda 2676.07$ ( $\log g f=-1.78$, according to the Kurucz \& Bell lists, which we adjust from -0.15 to 0.0 assuming a solar $[\mathrm{Ti} / \mathrm{Fe}]$ ratio). Laboratory $\log (g f)$ values are lacking for these transitions. If we assume that all absorption in HD 128279 is due to these three species, we can empirically fit the line profile, attributing the remaining absorption in HD 108317 to Au I. We advise that the derived abundance should be interpreted with due caution. The $\log (g f)$ values from Morton (2000) and Fivet et al. (2006) for this transition agree within 0.01 dex.

Lead $(Z=82)$. The $\mathrm{Pb}$ I $\lambda 2833.03$ line is blended with an Fe II line at $\lambda 2833.09$. NIST reports $\log (g f)=-0.48$ for this line with an uncertainty of $25 \%$. We fit this line profile well in HD 128279 assuming that none of the absorption is due to $\mathrm{Pb}$ I. Applying this fit directly to HD 108317 reveals that only a minimal amount of absorption at this wavelength may be due to $\mathrm{Pb}$ I, so we only derive an upper limit. The hfs/IS pattern for this transition is given in Appendix $\mathrm{C}$ along with three other $\mathrm{Pb}$ I lines commonly used for abundance analyses.

Other elements not detected. There is an unidentified absorption line at $\lambda 2943.58$ whose EWs vary from 20 to $70 \mathrm{~m} \AA$ in our sample. This is close to the predicted wavelength of a lowexcitation Ga I (gallium, $Z=31$ ) transition at $\lambda 2943.63$. We have searched for absorption from other zero- or low-excitation Ga I transitions at $2874.24,4032.98$, and $\lambda 4172.06$ that should be present if the absorption at $\lambda 2943.58$ is Ga I. We detect none of these lines. Gallium has a low first ionization potential of $6.00 \mathrm{eV}$ and should mostly be present in these stars as Ga II. We conclude that the absorption is not due to Ga I. We also synthesize regions around a number of low-excitation W I and II (tungsten, $Z=74$ ) lines, adopting $\log (g f)$ values from Kling $\&$ Kock (1999) and Morton (2000). None of these lines yield credible detections or interesting upper limits. We synthesize a region around the $\mathrm{Hg}$ I (mercury, $Z=80$ ) $\lambda 2536.52$ line, using the $\log (g f)$ value given by Morton (2000); unfortunately, this 
Table 11

Hyperfine Structure Line Component Pattern for ${ }^{175} \mathrm{Lu}$ II $\lambda 2615$

\begin{tabular}{|c|c|c|c|c|c|c|}
\hline $\begin{array}{l}\text { Wavenumber } \\
\left(\mathrm{cm}^{-1}\right)\end{array}$ & $\begin{array}{l}\lambda_{\text {air }} \\
(\AA)\end{array}$ & $F_{\text {upper }}$ & $F_{\text {lower }}$ & $\begin{array}{l}\text { Component Position } \\
\left(\mathrm{cm}^{-1}\right)\end{array}$ & $\begin{array}{c}\text { Component Position } \\
(\AA)\end{array}$ & Strength \\
\hline 38223.406 & 2615.4173 & 4.5 & 3.5 & -0.11034 & +0.007550 & 0.41667 \\
\hline 38223.406 & 2615.4173 & 3.5 & 3.5 & -0.02055 & +0.001406 & 0.33333 \\
\hline 38223.406 & 2615.4173 & 2.5 & 3.5 & +0.21129 & -0.014458 & 0.25000 \\
\hline
\end{tabular}

Table 12

Hyperfine Structure and Isotopic Line Component Patterns for $\mathrm{Pb}$ I Lines

\begin{tabular}{|c|c|c|c|c|c|c|c|}
\hline $\begin{array}{l}\text { Wavenumber } \\
\left(\mathrm{cm}^{-1}\right)\end{array}$ & $\begin{array}{l}\lambda_{\text {air }} \\
(\AA)\end{array}$ & $F_{\text {upper }}$ & $F_{\text {lower }}$ & $\begin{array}{l}\text { Component Position } \\
\qquad\left(\mathrm{cm}^{-1}\right)\end{array}$ & $\begin{array}{c}\text { Component Position } \\
(\AA)\end{array}$ & Strength & Isotope \\
\hline 35287.2545 & 2833.05104 & 1.0 & 0.0 & -0.11038 & +0.008862 & 0.014 & 204 \\
\hline 35287.2545 & 2833.05104 & 1.5 & 0.5 & +0.13053 & -0.010480 & 0.147 & 207 \\
\hline 35287.2545 & 2833.05104 & 0.5 & 0.5 & -0.30988 & +0.024880 & 0.074 & 207 \\
\hline 35287.2545 & 2833.05104 & 1.0 & 0.0 & +0.03012 & -0.002418 & 0.524 & 208 \\
\hline 27467.9949 & 3639.56335 & 1.0 & 1.0 & -0.04856 & +0.006435 & 0.241 & 206 \\
\hline 27467.9949 & 3639.56335 & 1.5 & 0.5 & +0.04930 & -0.006533 & 0.025 & 207 \\
\hline 27467.9949 & 3639.56335 & 1.5 & 1.5 & +0.16886 & -0.022374 & 0.123 & 207 \\
\hline 27467.9949 & 3639.56335 & 0.5 & 0.5 & -0.39110 & +0.051824 & 0.049 & 207 \\
\hline 27467.9949 & 3639.56335 & 0.5 & 1.5 & -0.27155 & +0.035982 & 0.025 & 207 \\
\hline 27467.9949 & 3639.56335 & 1.0 & 1.0 & +0.03308 & -0.004383 & 0.524 & 208 \\
\hline 27140.6795 & 3683.45748 & 0.0 & 1.0 & -0.12332 & +0.016737 & 0.014 & 204 \\
\hline 27140.6795 & 3683.45748 & 0.5 & 0.5 & -0.09792 & +0.013290 & 0.074 & 207 \\
\hline 27140.6795 & 3683.45748 & 0.5 & 1.5 & +0.02163 & -0.002935 & 0.147 & 207 \\
\hline 27140.6795 & 3683.45748 & 0.0 & 1.0 & +0.03369 & -0.004572 & 0.524 & 208 \\
\hline 24636.9301 & 4057.80118 & 1.0 & 2.0 & -0.12020 & +0.019798 & 0.014 & 204 \\
\hline 24636.9301 & 4057.80118 & 1.0 & 2.0 & -0.04815 & +0.007931 & 0.241 & 206 \\
\hline 24636.9301 & 4057.80118 & 1.5 & 2.5 & +0.04233 & -0.006973 & 0.133 & 207 \\
\hline 24636.9301 & 4057.80118 & 1.5 & 1.5 & +0.25922 & -0.042695 & 0.015 & 207 \\
\hline 24636.9301 & 4057.80118 & 0.5 & 1.5 & -0.18119 & +0.029843 & 0.074 & 207 \\
\hline 24636.9301 & 4057.80118 & 1.0 & 2.0 & +0.03283 & -0.005407 & 0.524 & 208 \\
\hline
\end{tabular}

line is far too blended to yield an interesting upper limit. We derive an upper limit on the bismuth abundance from the Bi I transition at $\lambda 3024.64$, adopting the $\log (g f)$ value given in the NIST database.

\section{APPENDIX B}

\section{NEW HFS PATTERNS FOR IONIZED LUTETIUM}

In Table 11 we present the hfs line component pattern for the $\mathrm{Lu}$ II $\lambda 2615$ transition. There are two stable isotopes of lutetium, ${ }^{175} \mathrm{Lu}$ and ${ }^{176} \mathrm{Lu}$. The ${ }^{175} \mathrm{Lu}$ isotope is dominant $(97.4 \%$ of SS lutetium; Böhlke et al. 2005). The ${ }^{176} \mathrm{Lu}$ isotope is blocked from $r$-process production by the stable ${ }^{176} \mathrm{Yb}$ isotope, so we expect that the lutetium in our sample of stars is primarily ${ }^{175} \mathrm{Lu}$. The odd- $Z$ isotope ${ }^{175} \mathrm{Lu}$ has non-zero nuclear spin $I=7 / 2$, which gives rise to the hfs. The format of Table 11 is the same as Table 14 of Lawler et al. (2009), which contains hfs component patterns for several other Lu II lines throughout the optical and NUV. These data were used previously to derive the Lu II abundance in BD +17 3248 and HD 122563 (Roederer et al. 2010b). The component positions are given relative to the center-of-gravity wavenumbers and air wavelengths. Strengths are normalized to sum to one. The positions are computed from the hfs constants given in Table 10 of Sneden et al. (2003), the energy levels given in Table 12 of Lawler et al. (2009), and the standard index of air given in Peck \& Reeder (1972).

\section{APPENDIX C}

\section{NEW HFS PATTERNS FOR NEUTRAL LEAD}

In Table 12 we present the hfs/IS line component pattern for four $\mathrm{Pb}$ I lines commonly used for abundance analysis. There are four stable isotopes of lead, ${ }^{204} \mathrm{~Pb},{ }^{206} \mathrm{~Pb},{ }^{207} \mathrm{~Pb}$, and ${ }^{208} \mathrm{~Pb}$. The odd- $Z$ isotope ${ }^{207} \mathrm{~Pb}$ has non-zero nuclear spin $I=1 / 2$, which gives rise to the hfs. The component positions are given relative to the center-of-gravity wavenumbers and air wavelengths. Strengths are normalized to sum to one for an SS isotopic composition, where the fraction of each isotope, $f$, is $f_{204} / f_{206} / f_{207} / f_{208}=0.014 / 0.241 / 0.221 / 0.524$ (Böhlke et al. 2005). Energy levels are adopted from Wood \& Andrew (1968). The hfs $A$ values are adopted from Bouazza et al. (2000) for all lines. The IS are adopted from Bouazza et al. for the $\lambda \lambda 3639$, 3683, and 4057 lines and Thompson et al. (1983) for the $\lambda 2833$ line. The standard index of air is given in Peck \& Reeder (1972).

\section{REFERENCES}

Alonso, A., Arribas, S., \& Martínez-Roger, C. 1999a, A\&AS, 139, 335 Alonso, A., Arribas, S., \& Martínez-Roger, C. 1999b, A\&AS, 140, 261 Alvarez, R., \& Plez, B. 1998, A\&A, 330, 1109

Anders, E. 1971, Geochim. Cosmochim. Acta, 35, 516

Anders, E., \& Grevesse, N. 1989, Geochim. Cosmochim. Acta, 53, 197

Aoki, W., Honda, S., Beers, T. C., et al. 2005, ApJ, 632, 611

Argast, D., Samland, M., Thielemann, F.-K., \& Qian, Y.-Z. 2004, A\&A, 416, 997

Arlandini, C., Käppeler, F., Wisshak, K., et al. 1999, ApJ, 525, 886 
Asplund, M., Grevesse, N., Sauval, A. J., \& Scott, P. 2009, ARA\&A, 47, 481

Barbuy, B., Spite, M., Hill, V., et al. 2011, A\&A, 534, A60

Barklem, P. S., \& Aspelund-Johansson, J. 2005, A\&A, 435, 373

Barklem, P. S., Christlieb, N., Beers, T. C., et al. 2005, A\&A, 439, 129

Barklem, P. S., Piskunov, N., \& O’Mara, B. J. 2000, A\&AS, 142, 467

Bernstein, R., Shectman, S. A., Gunnels, S. M., Mochnacki, S., \& Athey, A. E. 2003, Proc. SPIE, 4841, 1694

Biémont, É., Blagoev, K., Engström, L., et al. 2011, MNRAS, 414, 3350

Biémont, E., Garnir, H. P., Palmeri, P., Li, Z. S., \& Svanberg, S. 2000, MNRAS, 312,116

Bisterzo, S., Gallino, R., Straniero, O., Cristallo, S., \& Käppeler, F. 2010, MNRAS, 404, 1529

Bisterzo, S., Gallino, R., Straniero, O., Cristallo, S., \& Käppeler, F. 2011, MNRAS, 418, 284

Bohlin, R. C., Savage, B. D., \& Drake, J. F. 1978, ApJ, 224, 132

Böhlke, J. K., de Laeter, J. R., De Biévre, P., et al. 2005, J. Phys. Chem. Ref. Data, 34, 57

Bonifacio, P., Monai, S., \& Beers, T. C. 2000, AJ, 120, 2065

Bouazza, S., Gough, D. S., Hannaford, P., Lowe, R. M., \& Wilson, M. 2000, Phys. Rev. A, 63, 012516

Burris, D. L., Pilachowski, C. A., Armandroff, T. E., et al. 2000, ApJ, 544, 302

Busso, M., Gallino, R., \& Wasserburg, G. J. 1999, ARA\&A, 37, 239

Cameron, A. G. W. 1973, Space Sci. Rev., 15, 121

Cameron, A. G. W. 1982, Ap\&SS, 82, 123

Cardelli, J. A., Clayton, G. C., \& Mathis, J. S. 1989, ApJ, 345, 245

Castelli, F., \& Kurucz, R. L. 2003, in IAU Symp. 210, Modelling of Stellar Atmospheres, ed. N. Piskunov et al. (Cambridge: Cambridge Univ. Press), A20

Cohen, J. G., Christlieb, N., McWilliam, A., et al. 2008, ApJ, 672, 320

Cowan, J. J., Burris, D. L., Sneden, C., McWilliam, A., \& Preston, G. W. 1995, ApJ, 439, L51

Cowan, J. J., Sneden, C., Beers, T. C., et al. 2005, ApJ, 627, 238

Cowan, J. J., Sneden, C., Burles, S., et al. 2002, ApJ, 572, 861

Cowan, J. J., Sneden, C., Truran, J. W., \& Burris, D. L. 1996, ApJ, 460, L115

Creevey, O. L., Thévenin, F., Boyajian, T. S., et al. 2012, A\&A, 545, A17

Däppen, W., Anderson, L., \& Mihalas, D. 1987, ApJ, 319, 195

Den Hartog, E. A., Herd, M. T., Lawler, J. E., et al. 2005, ApJ, 619, 639

Den Hartog, E. A., Lawler, J. E., Sneden, C., \& Cowan, J. J. 2003, ApJS, 148, 543

Den Hartog, E. A., Lawler, J. E., Sneden, C., \& Cowan, J. J. 2006, ApJS, 167, 292

Duquette, D. W., \& Lawler, J. E. 1985, J. Opt. Soc. Am. B, 1, 1948

Farouqi, K., Kratz, K.-L., Pfeiffer, B., et al. 2010, ApJ, 712, 1359

Ferlet, R., Vidal-Madjar, A., \& Gry, C. 1985, ApJ, 298, 838

Fischer, T., Whitehouse, S. C., Mezzacappa, A., Thielemann, F.-K., \& Liebendörfer, M. 2010, A\&A, 517, A80

Fivet, V., Quinet, P., Biémont, É., \& Xu, H. L. 2006, J. Phys. B: At. Mol. Phys., 39,3587

François, P., Depagne, E., Hill, V., et al. 2007, A\&A, 476, 935

Frebel, A., Aoki, W., Christlieb, N., et al. 2005, Nature, 434, 871

Frebel, A., Christlieb, N., Norris, J. E., et al. 2007, ApJ, 660, L117

Frebel, A., Kirby, E. N., \& Simon, J. D. 2010a, Nature, 464, 72

Frebel, A., Simon, J. D., Geha, M., \& Willman, B. 2010b, ApJ, 708, 560

Freiburghaus, C., Rosswog, S., \& Thielemann, F.-K. 1999, ApJ, 525, L121

Fuhr, J. R., \& Wiese, W. L. 2006, J. Phys. Chem. Ref. Data, 35, 1669

Fuhr, J. R., \& Wiese, W. L. 2009, in CRC Handbook of Chemistry and Physics, ed. D. R. Lide (90th ed.; Boca Raton, FL: CRC Press), 10

Fulbright, J. P., Rich, R. M., \& Castro, S. 2004, ApJ, 612, 447

Gallino, R., Arlandini, C., Busso, M., et al. 1998, ApJ, 497, 388

Goriely, S., Bauswein, A., \& Janka, H.-T. 2011, ApJ, 738, L32

Hansen, C. J., \& Primas, F. 2011, A\&A, 525, L5

Hansen, C. J., Primas, F., Hartman, H., et al. 2012, A\&A, 545, A31

Hansen, T., Andersen, J., Nordström, B., Buchhave, L. A., \& Beers, T. C. 2011, ApJ, 743, L1

Hill, V., Plez, B., Cayrel, R., et al. 2002, A\&A, 387, 560

Hinkle, K., Wallace, L., Valenti, J., \& Ayres, T. (ed.) 2005, in Ultraviolet Atlas of the Arcturus Spectrum, 1150-3800 ̊̊ (San Francisco, CA: ASP)

Hinkle, K., Wallace, L., Valenti, J., \& Harmer, D. (ed.) 2000, Visible and Near Infrared Atlas of the Arcturus Spectrum 3727-9300 ̊ (San Francisco, CA: ASP)

Hollek, J. K., Frebel, A., Roederer, I. U., et al. 2011, ApJ, 742, 54

Honda, S., Aoki, W., Ishimaru, Y., \& Wanajo, S. 2007, ApJ, 666, 1189

Honda, S., Aoki, W., Ishimaru, Y., Wanajo, S., \& Ryan, S. G. 2006, ApJ, 643, 1180

Honda, S., Aoki, W., Kajino, T., et al. 2004, ApJ, 607, 474

Horowitz, C. J., \& Li, G. 1999, Phys. Rev. Lett., 82, 5198

Hubeny, I., Hummer, D. G., \& Lanz, T. 1994, A\&A, 282, 151
Hüdepohl, L., Müller, B., Janka, H.-T., Marek, A., \& Raffelt, G. G. 2010, Phys. Rev. Lett., 104, 251101

Hummer, D. G., \& Mihalas, D. 1988, ApJ, 331, 794

Ivans, I. I., Simmerer, J., Sneden, C., et al. 2006, ApJ, 645, 613

Ivarsson, S., Wahlgren, G. M., Dai, Z., Lundberg, H., \& Leckrone, D. S 2004, A\&A, 425, 353

Janka, H.-T., Müller, B., Kitaura, F. S., \& Buras, R. 2008, A\&A, 485, 199

Johnson, J. A., \& Bolte, M. 2002, ApJ, 579, 616

Käppeler, F., Beer, H., \& Wisshak, K. 1989, Rep. Prog. Phys., 52, 945

Karzas, W. J., \& Latter, R. 1961, ApJS, 6, 167

Kelson, D. D. 2003, PASP, 115, 688

Kimble, R. A., Woodgate, B. E., Bowers, C. W., et al. 1998, ApJ, 492, L83

Kling, R., \& Kock, M. 1999, J. Quant. Spectrosc. Radiat. Transfer, 62, 129

Koch, A., McWilliam, A., Grebel, E. K., Zucker, D. B., \& Belokurov, V. 2008, ApJ, 688, L13

Kratz, K.-L., Farouqi, K., Pfeiffer, B., et al. 2007, ApJ, 662, 39

Kurucz, R. L. 1970, Atlas: A Computer Program for Calculating Model Stellar Atmospheres, SAO Special Report 309 (Cambridge, MA: Smithsonian Astrophysical Observatory)

Kurucz, R. L., \& Bell, B. 1995, Kurucz CD-ROM 23 (Cambridge, MA: Smithsonian Astrophysical Observatory)

Kwiatkowski, M., Zimmermann, P., Biémont, E., \& Grevesse, N. 1982, A\&A, 112,337

Lai, D. K., Bolte, M., Johnson, J. A., et al. 2008, ApJ, 681, 1524

Lawler, J. E., Bonvallet, G., \& Sneden, C. 2001a, ApJ, 556, 452

Lawler, J. E., Den Hartog, E. A., Labby, Z. E., et al. 2007, ApJS, 169, 120

Lawler, J. E., Den Hartog, E. A., Sneden, C., \& Cowan, J. J. 2006, ApJS, 162, 227

Lawler, J. E., Sneden, C., \& Cowan, J. J. 2004, ApJ, 604, 850

Lawler, J. E., Sneden, C., Cowan, J. J., Ivans, I. I., \& Den Hartog, E. A. 2009, ApJS, 182, 51

Lawler, J. E., Sneden, C., Cowan, J. J., et al. 2008, ApJS, 178, 71

Lawler, J. E., Wickliffe, M. E., Cowley, C. R., \& Sneden, C. 2001b, ApJS, 137, 341

Lawler, J. E., Wickliffe, M. E., den Hartog, E. A., \& Sneden, C. 2001c, ApJ, 563,1075

Leckrone, D. S., Proffitt, C. R., Wahlgren, G. M., Johansson, S. G., \& Brage, T. 1999, AJ, 117, 1454

Ljung, G., Nilsson, H., Asplund, M., \& Johansson, S. 2006, A\&A, 456, 1181

Li, R., Chatelain, R., Holt, R. A., et al. 2007, Phys. Scr., 76, 577

Lugaro, M., Karakas, A. I., Stancliffe, R. J., \& Rijs, C. 2012, ApJ, 747, 2

Malcheva, G., Blagoev, K., Mayo, R., et al. 2006, MNRAS, 367, 754

Mashonkina, L., Ryabtsev, A., \& Frebel, A. 2012, A\&A, 540, A98

Mathews, G. J., Bazan, G., \& Cowan, J. J. 1992, ApJ, 391, 719

McClure, R. D. 1984, ApJ, 280, L31

McClure, R. D., \& Woodsworth, A. W. 1990, ApJ, 352, 709

McWilliam, A. 1998, AJ, 115, 1640

McWilliam, A., Preston, G. W., Sneden, C., \& Searle, L. 1995, AJ, 109, 2757

Migdalek, J., \& Baylis, W. E. 1987, Can. J. Phys., 65, 1612

Montes, F., Beers, T. C., Cowan, J., et al. 2007, ApJ, 671, 1685

Morton, D. C. 2000, ApJS, 130, 403

Nilsson, H., Hartman, H., Engström, L., et al. 2010, A\&A, 511, A16

Nilsson, H., \& Ivarsson, S. 2008, A\&A, 492, 609

Nilsson, H., Zhang, Z. G., Lundberg, H., Johansson, S., \& Nordström, B 2002, A\&A, 382, 368

Peck, E. R., \& Reeder, K. 1972, J. Opt. Soc. Am., 62, 958

Perryman, M. A. C., Lindegren, L., Kovalevsky, J., et al. 1997, A\&A, 323, L49

Peterson, R. C. 2011, ApJ, 742, 21

Qian, Y.-Z., \& Wasserburg, G. J. 2007, Phys. Rep., 442, 237

Qian, Y.-Z., \& Wasserburg, G. J. 2008, ApJ, 687, 272

Quinet, P., Palmeri, P., Biémont, É., et al. 2006, A\&A, 448, 1207

Raiteri, C.-M., Gallino, R., Busso, M., Neuberger, D., \& Käppeler, F. 1993, ApJ, 419, 207

Ralchenko, Yu., Kramida, A., Reader, J., et al. 2011, NIST Atomic Spectra Database, version 4.1, http://physics.nist.gov/asd

Roberts, L. F., Woosley, S. E., \& Hoffman, R. D. 2010, ApJ, 722, 954

Roederer, I. U. 2011, ApJ, 732, L17

Roederer, I. U. 2012a, ApJ, 756, 36

Roederer, I. U. 2012b, AJ, submitted

Roederer, I. U., Cowan, J. J., Karakas, A. I., et al. 2010a, ApJ, 724, 975

Roederer, I. U., Kratz, K.-L., Frebel, A., et al. 2009, ApJ, 698, 1963

Roederer, I. U., \& Lawler, J. E. 2012, ApJ, 750, 76

Roederer, I. U., Lawler, J. E., Cowan, J. J., et al. 2012, ApJ, 747, L8

Roederer, I. U., Lawler, J. E., Sneden, C., et al. 2008, ApJ, 675, 723

Roederer, I. U., Sneden, C., Lawler, J. E., \& Cowan, J. J. 2010b, ApJ, 714, L123

Roederer, I. U., Sneden, C., Thompson, I. B., Preston, G. W., \& Shectman, S. A. 2010c, ApJ, 711, 573 
Ryan, S. G., Norris, J. E., \& Beers, T. C. 1996, ApJ, 471, 254

Schlegel, D. J., Finkbeiner, D. P., \& Davis, M. 1998, ApJ, 500, 525

Seeger, P. A., Fowler, W. A., \& Clayton, D. D. 1965, ApJS, 11, 121

Sikström, C. M., Pihlemark, H., Nilsson, H., et al. 2001, J. Phys. B: At. Mol. Phys., 34, 477

Skrutskie, M. F., Cutri, R. M., Stiening, R., et al. 2006, AJ, 131, 1163

Sneden, C., Cowan, J. J., Burris, D. L., \& Truran, J. W. 1998, ApJ, 496, 235

Sneden, C., Cowan, J. J., \& Gallino, R. 2008, ARA\&A, 46, 241

Sneden, C., Cowan, J. J., Ivans, I. I., et al. 2000, ApJ, 533, L139

Sneden, C., Cowan, J. J., Lawler, J. E., et al. 2003, ApJ, 591, 936

Sneden, C., Lawler, J. E., Cowan, J. J., Ivans, I. I., \& Den Hartog, E. A. 2009, ApJS, 182, 80

Sneden, C., \& Parthasarathy, M. 1983, ApJ, 267, 757

Sneden, C. A. 1973, PhD thesis, Univ. Texas at Austin

Sobeck, J. S., Kraft, R. P., Sneden, C., et al. 2011, AJ, 141, 175

Thompson, R. C., Anselment, M., Bekk, K., et al. 1983, J. Phys. G: Nucl. Phys., 9, 443

Travaglio, C., Gallino, R., Arnone, E., et al. 2004, ApJ, 601, 864

Truran, J. W., Cowan, J. J., Pilachowski, C. A., \& Sneden, C. 2002, PASP, 114, 1293

Unsöld, A. 1955, Physik der Sternatmosphären (Berlin: Springer), 332 van Leeuwen, F. 2007, A\&A, 474, 653

Vogt, S. S., Allen, S. L., Bigelow, B. C., et al. 1994, Proc. SPIE, 2198, 362

Wanajo, S., \& Ishimaru, Y. 2006, Nucl. Phys. A, 777, 676

Wanajo, S., Tamamura, M., Itoh, N., et al. 2003, ApJ, 593, 968

Wasserburg, G. J., Busso, M., \& Gallino, R. 1996, ApJ, 466, L109

Whaling, W., \& Brault, J. W. 1988, Phys. Scr., 38, 707

Wheeler, J. C., Cowan, J. J., \& Hillebrandt, W. 1998, ApJ, 493, L101

Wickliffe, M. E., \& Lawler, J. E. 1997, J. Opt. Soc. Am. B, 14, 737

Wickliffe, M. E., Lawler, J. E., \& Nave, G. 2000, J. Quant. Spectrosc. Radiat. Transfer, 66, 363

Wickliffe, M. E., Salih, S., \& Lawler, J. E. 1994, J. Quant. Spectrosc. Radiat. Transfer, 51, 545

Wood, D. R., \& Andrew, K. L. 1968, J. Opt. Soc. Am., 58, 818

Woodgate, B. E., Kimble, R. A., Bowers, C. W., et al. 1998, PASP, 110,1183

Woosley, S. E., Wilson, J. R., Mathews, G. J., Hoffman, R. D., \& Meyer, B. S. 1994, ApJ, 433, 229

Xu, H. L., Sun, Z. W., Dai, Z. W., et al. 2006, A\&A, 452, 357

Xu, H. L., Svanberg, S., Quinet, P., Palmeri, P., \& Biémont, É. 2007, J. Quant. Spectrosc. Radiat. Transfer, 104, 52

Zheng, W., Proffit, C., \& Sahnow, D. 2011, Instrument Science Report STIS 2011-03, 1 